Supporting Information for

\title{
Design, Synthesis and Antifungal Activity of Novel Thiophene/Furan-1,3,4-Oxadiazole Carboxamides as Potent Succinate Dehydrogenase inhibitors
}

Zihui Yang, ${ }^{\dagger}$ Yue Sun ${ }^{\dagger}$ Qingsong Liu, Aliang Li, Wenyan Wang, and Wen $\mathrm{Gu}^{*}$

Jiangsu Provincial Key Lab for the Chemistry and Utilization of Agro-forest Biomass, Jiangsu

Key Lab of Biomass-based Green Fuels and Chemicals, College of Chemical Engineering,

Nanjing Forestry University, Nanjing 210037, China

*Corresponding author. E-mail: njguwen@163.com

$\dagger$ These two authors contributed equally to this paper. 


\section{Content}

Detailed data of $\mathbf{3 a}, \mathbf{3 b}$ and compounds $\mathbf{4 a - 4 o}$ and compounds $\mathbf{5 a - 5 o \ldots \ldots . . . 1}$

${ }^{1} \mathrm{H}$ NMR and ${ }^{13} \mathrm{C}$ NMR spectra of compound 3a, 3b, 4a-4o and 5a-5o

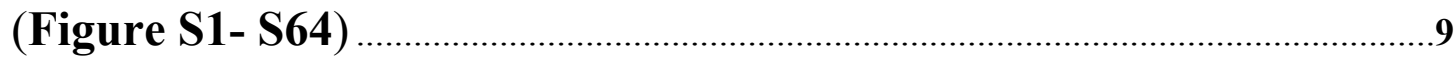

Crystallographic data of compound $4 \mathrm{~g}$ (Table S1) .........................................41 

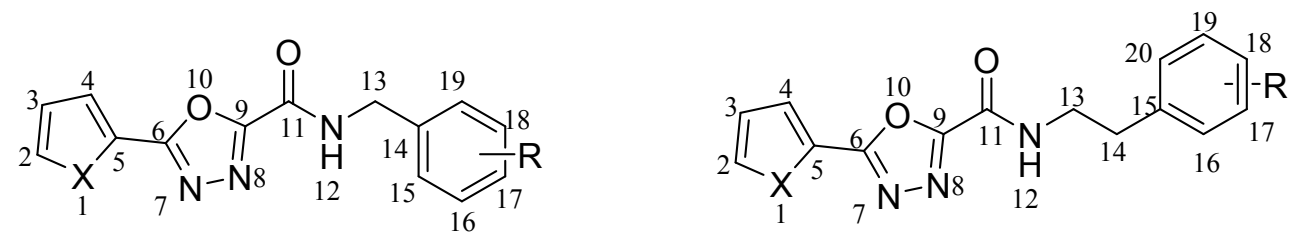

$4 a-40,5 a-5 o$

1. Detailed data of 3a, 3b and compounds $4 \mathbf{a}-\mathbf{4 o}$ and compounds $\mathbf{5 a - 5 o}$.

Data for 3a. Yellow solid; yield, 85\%, m.p. 122-124 ${ }^{\circ} \mathrm{C} ;{ }^{1} \mathrm{H}$ NMR (600 MHz, DMSO- $\left.d_{6}\right): \delta 8.06\left(\mathrm{dd}, J_{1}=5.0 \mathrm{~Hz}, J_{2}=1.1 \mathrm{~Hz}, 1 \mathrm{H}, \mathrm{H}-4\right.$, thiophene), 7.97 (dd, $J_{1}=$ 3.7 Hz, $J_{2}=1.1 \mathrm{~Hz}, 1 \mathrm{H}, \mathrm{H}-2$, thiophene), $7.35\left(\mathrm{dd}, J_{1}=5.0 \mathrm{~Hz}, J_{2}=3.8 \mathrm{~Hz}, 1 \mathrm{H}, \mathrm{H}-3\right.$, thiophene), $3.98\left(\mathrm{~s}, 3 \mathrm{H}, \mathrm{COOCH}{ }_{3}\right) \cdot{ }^{13} \mathrm{C} \mathrm{NMR}\left(150 \mathrm{MHz}, \mathrm{DMSO}-d_{6}\right): \delta 161.67(\mathrm{C}=\mathrm{O})$, $155.72,154.33,133.18,131.81,129.10,123.28,53.54$.

Data for 3b. Yellow solid; yield, 90\%, m.p. 130-131 ${ }^{\circ} \mathrm{C}$; ${ }^{1} \mathrm{H}$ NMR $(600 \mathrm{MHz}$, DMSO- $\left.d_{6}\right): \delta 8.15(\mathrm{~d}, J=1.1 \mathrm{~Hz}, 1 \mathrm{H}, \mathrm{H}-2$, furan), $7.55(\mathrm{~d}, J=3.6 \mathrm{~Hz}, 1 \mathrm{H}, \mathrm{H}-4$, furan), $6.86\left(\mathrm{dd}, J_{1}=3.6 \mathrm{~Hz}, J_{2}=1.7 \mathrm{~Hz}, 1 \mathrm{H}, \mathrm{H}-3\right.$, furan $), 3.98\left(\mathrm{~s}, 3 \mathrm{H}, \mathrm{COOCH}_{3}\right) .{ }^{13} \mathrm{C} \mathrm{NMR}$ $\left(150 \mathrm{MHz}, \mathrm{DMSO}-d_{6}\right): \delta 158.00(\mathrm{C}=\mathrm{O}), 155.53,154.30,148.02,137.85,116.49$, $113.01,53.56$.

Data for 4a. White solid; yield, 53.3\%, m.p. $184-186{ }^{\circ} \mathrm{C} ;{ }^{1} \mathrm{H}$ NMR $(600 \mathrm{MHz}$, DMSO-d6): $\delta 9.91(\mathrm{t}, J=6.1 \mathrm{~Hz}, 1 \mathrm{H}, \mathrm{CONH}), 8.03(\mathrm{~d}, J=4.9 \mathrm{~Hz}, 1 \mathrm{H}, \mathrm{H}-4$, thiophene), $7.93(\mathrm{~d}, J=3.6 \mathrm{~Hz}, 1 \mathrm{H}, \mathrm{H}-2$, thiophene), $7.41(\mathrm{~d}, J=8.6 \mathrm{~Hz}, 2 \mathrm{H}$, H-16,18), 7.39 (d, $J=8.6 \mathrm{~Hz}, 2 \mathrm{H}, \mathrm{H}-15,19), 7.33$ (t, $J=4.0 \mathrm{~Hz}, 1 \mathrm{H}, \mathrm{H}-3$, thiophene), $4.48\left(\mathrm{~d}, J=6.2 \mathrm{~Hz}, 2 \mathrm{H}, \mathrm{NHCH}_{2}\right) \cdot{ }^{13} \mathrm{C}$ NMR $\left(150 \mathrm{MHz}, \mathrm{DMSO}-d_{6}\right): \delta 161.36(\mathrm{C}=\mathrm{O})$, $157.78,153.04,137.38,132.69,131.62,131.42,129.33,128.95,128.26,123.58$, 41.89. ESI-HRMS: $m / z$ calcd. for $\mathrm{C}_{14} \mathrm{H}_{11} \mathrm{ClN}_{3} \mathrm{O}_{2} \mathrm{~S}[\mathrm{M}+\mathrm{H}]^{+}:$320.0261; found 320.0265 .

Data for 4b. White solid; yield, 62.3\%, m.p. 153-154 ${ }^{\circ} \mathrm{C}$; ${ }^{1} \mathrm{H}$ NMR (600 MHz, DMSO- $\left.d_{6}\right) \delta 9.89$ (t, $\left.J=6.0 \mathrm{~Hz}, 1 \mathrm{H}, \mathrm{CONH}\right), 8.03$ (d, $J=4.9 \mathrm{~Hz}, 1 \mathrm{H}, \mathrm{H}-4$, thiophene), $7.93\left(\mathrm{~d}, J=3.6 \mathrm{~Hz}, 1 \mathrm{H}, \mathrm{H}-2\right.$, thiophene), $7.40\left(\mathrm{dd}, J_{1}=8.2 \mathrm{~Hz}, J_{2}=5.8 \mathrm{~Hz}, 2 \mathrm{H}, \mathrm{H}-15\right.$, 19, phenyl), 7.33 (t, $J=4.2 \mathrm{~Hz}, 1 \mathrm{H}, \mathrm{H}-3$, thiophene), 7.17 (t, $J=8.8 \mathrm{~Hz}, 2 \mathrm{H}, \mathrm{H}-16,18$, 
phenyl), $4.47\left(\mathrm{~d}, J=6.2 \mathrm{~Hz}, 2 \mathrm{H}, \mathrm{NH}_{\underline{\mathrm{CH}}}\right) \cdot{ }^{13} \mathrm{C} \mathrm{NMR}\left(150 \mathrm{MHz}, \mathrm{DMSO}-d_{6}\right) \delta 161.41$ $(\mathrm{C}=\mathrm{O}), 161.34\left(\mathrm{~d},{ }^{1} J_{\mathrm{C}-\mathrm{F}}=241.3 \mathrm{~Hz}, \mathrm{C}-17\right), 157.85,153.04,134.58\left(\mathrm{~d},{ }^{4} J_{\mathrm{C}-\mathrm{F}}=2.9 \mathrm{~Hz}\right.$, C-14), 133.22, 131.72, 131.48, 129.57 (d, $\left.{ }^{3} J_{\mathrm{C}-\mathrm{F}}=8.2 \mathrm{~Hz}, \mathrm{C}-15,19\right), 129.01,123.62$, $115.10\left(\mathrm{~d},{ }^{2} J_{\mathrm{C}-\mathrm{F}}=21.2 \mathrm{~Hz}, \mathrm{C}-16,18\right), 41.89$. ESI-HRMS: $m / z$ calcd. for $\mathrm{C}_{14} \mathrm{H}_{11} \mathrm{FN}_{3} \mathrm{O}_{2} \mathrm{~S}$ $[\mathrm{M}+\mathrm{H}]^{+}:$304.0556; found 304.0558.

Data for 4c. White powder; yield 55.6\%, m.p. 137-139 ${ }^{\circ} \mathrm{C} ;{ }^{1} \mathrm{H}$ NMR $(600 \mathrm{MHz}$, DMSO- $\left.d_{6}\right): \delta 9.83(\mathrm{t}, J=6.2 \mathrm{~Hz}, 1 \mathrm{H}, \mathrm{CONH}), 8.01\left(\mathrm{dd}, J_{1}=5.0 \mathrm{~Hz}, J_{2}=1.2 \mathrm{~Hz}, 1 \mathrm{H}\right.$, $\mathrm{H}-4$, thiophene), $7.92\left(\mathrm{dd}, J_{1}=3.7 \mathrm{~Hz}, J_{2}=1.2 \mathrm{~Hz}, 1 \mathrm{H}, \mathrm{H}-2\right.$, thiophene), 7.35 (d, $J=$ $8.4 \mathrm{~Hz}, 2 \mathrm{H}, \mathrm{H}-16,18$, phenyl), 7.32 (dd, $J_{1}=5.0 \mathrm{~Hz}, J_{2}=3.7 \mathrm{~Hz}, 1 \mathrm{H}, \mathrm{H}-3$, thiophene), 7.27 (d, $J=8.4 \mathrm{~Hz}, 2 \mathrm{H}, \mathrm{H}-15,19$, phenyl), 4.43 (d, $\left.J=6.2 \mathrm{~Hz}, 2 \mathrm{H}, \mathrm{NHCH}_{2}\right), 1.25$ (s, 9H,4-C(C( $\left.\left.\mathrm{CH}_{3}\right)_{3}\right) .{ }^{13} \mathrm{C}$ NMR (150 MHz, DMSO- $\left.d_{6}\right): \delta 161.32(\mathrm{C}=\mathrm{O}), 157.85,152.87$, 149.45, 135.34, 132.65, 131.40, 128.93, 127.32, 125.04, 123.59, 42.23, 34.14, 31.11. ESI-HRMS: $m / z$ calcd. for $\mathrm{C}_{18} \mathrm{H}_{20} \mathrm{~N}_{3} \mathrm{O}_{2} \mathrm{~S}[\mathrm{M}+\mathrm{H}]^{+}:$342.1276; found 342.1274.

Data for 4d. White powder; yield, 64.7\%, m.p. 159-161 ${ }^{\circ} \mathrm{C} ;{ }^{1} \mathrm{H}$ NMR $(600 \mathrm{MHz}$, DMSO- $\left.d_{6}\right): \delta 9.83(\mathrm{t}, J=6.2 \mathrm{~Hz}, 1 \mathrm{H}, \mathrm{CONH}), 8.02\left(\mathrm{dd}, J_{1}=5.0 \mathrm{~Hz}, J_{2}=1.1 \mathrm{~Hz}, 1 \mathrm{H}\right.$, $\mathrm{H}-4$, thiophene), $7.93\left(\mathrm{dd}, J_{1}=3.7 \mathrm{~Hz}, J_{2}=1.1 \mathrm{~Hz}, 1 \mathrm{H}, \mathrm{H}-2\right.$, thiophene), 7.33 (dd, $J_{1}$ $=4.9 \mathrm{~Hz}, J_{2}=3.8 \mathrm{~Hz}, 1 \mathrm{H}, \mathrm{H}-3$, thiophene), 7.24 (d, J=7.9 Hz, 2H, H-15, 19, phenyl), 7.14 (d, $J=7.9 \mathrm{~Hz}, 2 \mathrm{H}, \mathrm{H}-16,18$, phenyl), 4.44 (d, $\left.J=6.2 \mathrm{~Hz}, 2 \mathrm{H}, \mathrm{NHCH}_{2}\right), 2.28$ (s, $\left.3 \mathrm{H}, \mathrm{CH}_{3}\right) .{ }^{13} \mathrm{C} \mathrm{NMR}\left(150 \mathrm{MHz}, \mathrm{DMSO}-d_{6}\right): \delta 161.32(\mathrm{C}=\mathrm{O}), 157.85,152.89,136.12$, 135.31, 132.65, 131.41, 128.93, 128.84, 127.44, 42.27, 20.64. ESI-HRMS: $m / z$ calcd. for $\mathrm{C}_{15} \mathrm{H}_{14} \mathrm{~N}_{3} \mathrm{O}_{2} \mathrm{~S}[\mathrm{M}+\mathrm{H}]^{+}:$300.0807; found 300.0812 .

Data for 4e. White powder; yield, 75.0\%, m.p. 166-168 ${ }^{\circ} \mathrm{C} ;{ }^{1} \mathrm{H}$ NMR $(600 \mathrm{MHz}$, DMSO- $\left.d_{6}\right): \delta 9.90(\mathrm{t}, J=6.1 \mathrm{~Hz}, 1 \mathrm{H}, \mathrm{CONH}), 8.03\left(\mathrm{dd}, J_{1}=5.0 \mathrm{~Hz}, J_{2}=1.0 \mathrm{~Hz}, 1 \mathrm{H}\right.$, $\mathrm{H}-4$, thiophene), 7.93 (dd, $J_{1}=3.7 \mathrm{~Hz}, J_{2}=1.1 \mathrm{~Hz}, 1 \mathrm{H}, \mathrm{H}-2$, thiophene), 7.41-7.37 (m, 1H, H-16, phenyl), 7.34 (dd, $J_{1}=4.9 \mathrm{~Hz}, J_{2}=3.8 \mathrm{~Hz}, 1 \mathrm{H}, \mathrm{H}-3$, thiophene), 7.19 (t, $J=$ $7.9 \mathrm{~Hz}, 2 \mathrm{H}, \mathrm{H}-17,19$, phenyl), 7.11-7.08 (m, 1H, H-15, phenyl), 4.51 (d, $J=6.2 \mathrm{~Hz}$, $\left.2 \mathrm{H}, \mathrm{NHCH}_{2}\right) \cdot{ }^{13} \mathrm{C} \mathrm{NMR}\left(150 \mathrm{MHz}, \mathrm{DMSO}-d_{6}\right): \delta 162.62\left(\mathrm{~d},{ }^{1} J_{\mathrm{C}-\mathrm{F}}=243.7 \mathrm{~Hz}, \mathrm{C}-18\right)$, $161.79(\mathrm{C}=\mathrm{O}), 158.24,153.65,141.67\left(\mathrm{~d},{ }^{3} J_{\mathrm{C}-\mathrm{F}}=7.1 \mathrm{~Hz}, \mathrm{C}-14\right), 133.10,131.85$, $130.70\left(\mathrm{~d},{ }^{3} J_{\mathrm{C}-\mathrm{F}}=7.9 \mathrm{~Hz}, \mathrm{C}-16\right), 129.49,124.05,123.80\left(\mathrm{~d},{ }^{4} J_{\mathrm{C}-\mathrm{F}}=1.7 \mathrm{~Hz}, \mathrm{C}-15\right)$, 
$114.52\left(\mathrm{~d},{ }^{2} J_{\mathrm{C}-\mathrm{F}}=21.4 \mathrm{~Hz}, \mathrm{C}-19\right), 114.22\left(\mathrm{~d},{ }^{2} J_{\mathrm{C}-\mathrm{F}}=20.6 \mathrm{~Hz}, \mathrm{C}-17\right), 42.57$. ESI-HRMS: $m / z$ calcd. for $\mathrm{C}_{14} \mathrm{H}_{11} \mathrm{FN}_{3} \mathrm{O}_{2} \mathrm{~S}[\mathrm{M}+\mathrm{H}]^{+}$: 304.0556; found 304.0553.

Data for 4f. White powder; yield, 83.1\%, m.p. 170-172 ${ }^{\circ} \mathrm{C} ;{ }^{1} \mathrm{H}$ NMR (600 MHz, DMSO- $\left.d_{6}\right): \delta 9.87(\mathrm{t}, J=5.8 \mathrm{~Hz}, 1 \mathrm{H}, \mathrm{CONH}), 8.03(\mathrm{~d}, J=4.9 \mathrm{~Hz}, 1 \mathrm{H}, \mathrm{H}-4$, thiophene), 7.93 (d, $J=3.7 \mathrm{~Hz}, 1 \mathrm{H}, \mathrm{H}-2$, thiophene), 7.43 (t, $J=7.7 \mathrm{~Hz}, 1 \mathrm{H}, \mathrm{H}-16$, phenyl), 7.33 (dd, $J_{1}=8.4 \mathrm{~Hz}, J_{2}=4.7 \mathrm{~Hz}, 2 \mathrm{H}, \mathrm{H}-3$, thiophene, H-18, phenyl), 7.22-7.17 (m, 2H, H-16,19, phenyl), 4.54 (d, $\left.J=5.9 \mathrm{~Hz}, 2 \mathrm{H}, \mathrm{NHCH}_{2}\right) .{ }^{13} \mathrm{C}$ NMR $(150$ MHz, DMSO- $\left.d_{6}\right): \delta 161.94(\mathrm{C}=\mathrm{O}), 160.52\left(\mathrm{~d},{ }^{1} J_{\mathrm{C}-\mathrm{F}}=243.4 \mathrm{~Hz}, \mathrm{C}-19\right), 159.71,158.30$, 153.68, 133.26, 132.02, $130.23\left(\mathrm{~d},{ }^{3} J_{\mathrm{C}-\mathrm{F}}=4.0 \mathrm{~Hz}, \mathrm{C}-15\right), 129.74\left(\mathrm{~d},{ }^{3} J_{\mathrm{C}-\mathrm{F}}=8.0 \mathrm{~Hz}\right.$, C-17), 129.52, $125.44\left(\mathrm{~d},{ }^{2} J_{\mathrm{C}-\mathrm{F}}=14.7 \mathrm{~Hz}, \mathrm{C}-14\right), 124.91\left(\mathrm{~d},{ }^{4} J_{\mathrm{C}-\mathrm{F}}=3.3 \mathrm{~Hz}, \mathrm{C}-16\right)$, 124.16, $115.73\left(\mathrm{~d},{ }^{2} J_{\mathrm{C}-\mathrm{F}}=20.8 \mathrm{~Hz}, \mathrm{C}-18\right), 36.96$ (d, $\left.J=4.6 \mathrm{~Hz}, \mathrm{C}-13\right)$. ESI-HRMS: $m / z$ calcd. for $\mathrm{C}_{14} \mathrm{H}_{11} \mathrm{FN}_{3} \mathrm{O}_{2} \mathrm{~S}[\mathrm{M}+\mathrm{H}]^{+}:$304.0556; found 304.0559 .

Data for 4g. White powder; yield, 41.0\%, m.p. 197-199 ${ }^{\circ} \mathrm{C} ;{ }^{1} \mathrm{H}$ NMR $(600 \mathrm{MHz}$, DMSO- $\left.d_{6}\right): \delta 9.97(\mathrm{t}, J=6.2 \mathrm{~Hz}, 1 \mathrm{H}, \mathrm{CONH}), 8.03\left(\mathrm{dd}, J_{1}=5.0 \mathrm{~Hz}, J_{2}=1.1 \mathrm{~Hz}, 1 \mathrm{H}\right.$, H-4, thiophene), 7.93 (dd, $J_{1}=3.7 \mathrm{~Hz}, J_{2}=1.2 \mathrm{~Hz}, 1 \mathrm{H}, \mathrm{H}-2$, thiophene), 7.71 (d, $J=$ $8.2 \mathrm{~Hz}, 2 \mathrm{H}, \mathrm{H}-15,19$, phenyl), 7.57 (d, $J=8.1 \mathrm{~Hz}, 2 \mathrm{H}, \mathrm{H}-16,18$, phenyl), 7.33 (dd, $J_{1}$ $=5.0 \mathrm{~Hz}, J_{2}=3.8 \mathrm{~Hz}, 1 \mathrm{H}, \mathrm{H}-3$, thiophene), 4.57 (d, $\left.J=6.2 \mathrm{~Hz}, 2 \mathrm{H}, \mathrm{NHCH}_{2}\right) .{ }^{13} \mathrm{C}$ NMR (150 MHz, DMSO- $\left.d_{6}\right): \delta 161.89(\mathrm{C}=\mathrm{O}), 158.24,153.66,143.67,133.21,131.94$, $129.46,128.59,128.20\left(\mathrm{q},{ }^{2} J_{\mathrm{C}-\mathrm{F}}=31.5 \mathrm{~Hz}, \mathrm{C}-17\right), 125.70\left(\mathrm{q},{ }^{3} J_{\mathrm{C}-\mathrm{F}}=3.5 \mathrm{~Hz}, \mathrm{C}-16,18\right)$, $123.46\left(\mathrm{q},{ }^{1} J_{\mathrm{C}-\mathrm{F}}=286.1 \mathrm{~Hz}, \mathrm{CF}_{3}\right), 124.07,42.69$. ESI-HRMS: $\mathrm{m} / \mathrm{z}$ calcd. for $\mathrm{C}_{15} \mathrm{H}_{11} \mathrm{~F}_{3} \mathrm{~N}_{3} \mathrm{O}_{2} \mathrm{~S}[\mathrm{M}+\mathrm{H}]^{+}:$354.0524; found 354.0519.

Data for 4h. Yellow powder; yield, 52.0\%, m.p. 159-161 ${ }^{\circ} \mathrm{C} ;{ }^{1} \mathrm{H}$ NMR $(600 \mathrm{MHz}$, DMSO- $\left.d_{6}\right): \delta 9.42(\mathrm{t}, J=5.7 \mathrm{~Hz}, 1 \mathrm{H}, \mathrm{CONH}), 8.04(\mathrm{~d}, J=6.1 \mathrm{~Hz}, 1 \mathrm{H}, \mathrm{H}-4$, thiophene), $7.94\left(\mathrm{~d}, J=4.8 \mathrm{~Hz}, 1 \mathrm{H}, \mathrm{H}-2\right.$, thiophene), 7.35 (dd, $\underline{J}_{1}=4.9 \mathrm{~Hz}, J_{2}=3.8 \mathrm{~Hz}, 1 \mathrm{H}, \mathrm{H}-3$, thiophene), 7.30 (dd, $J_{1}=8.5 \mathrm{~Hz}, J_{2}=5.7 \mathrm{~Hz}, 2 \mathrm{H}, \mathrm{H}-16,20$, phenyl), 7.13 (t, $J=8.9$ $\mathrm{Hz}, 2 \mathrm{H}, \mathrm{H}-17,19$, phenyl), 3.53 (q, $J=6.8 \mathrm{~Hz}, 2 \mathrm{H}, \mathrm{NHCH}_{2} \mathrm{CH}_{2}$ ), 2.88 (t, $J=7.3 \mathrm{~Hz}$, $\left.2 \mathrm{H}, \mathrm{NHCH}_{2} \mathrm{CH}_{2}\right) \cdot{ }^{13} \mathrm{C}$ NMR (150 MHz, DMSO-d 6$): \delta 161.82(\mathrm{C}=\mathrm{O}), 161.37\left({ }^{1} J_{\mathrm{C}-\mathrm{F}}=\right.$ $239.8 \mathrm{~Hz}, \mathrm{C}-18), 160.57,158.28\left(\mathrm{~d},{ }^{3} J_{\mathrm{C}-\mathrm{F}}=3.9 \mathrm{~Hz}, \mathrm{C}-16\right), 153.27,135.59\left(\mathrm{~d},{ }^{4} J_{\mathrm{C}-\mathrm{F}}=\right.$ $2.7 \mathrm{~Hz}, \mathrm{C}-15), 133.17,131.90,130.95\left(\mathrm{~d},{ }^{3} J_{\mathrm{C}-\mathrm{F}}=7.9 \mathrm{~Hz}, \mathrm{C}-20\right), 129.45,124.06$, 
115.51(d, $\left.{ }^{2} J_{\mathrm{C}-\mathrm{F}}=20.9 \mathrm{~Hz}, \mathrm{C}-17,19\right), 41.09,34.14$. ESI-HRMS: $m / z$ calcd. for $\mathrm{C}_{15} \mathrm{H}_{13} \mathrm{FN}_{3} \mathrm{O}_{2} \mathrm{~S}[\mathrm{M}+\mathrm{H}]^{+}:$318.0713; found 318.0718.

Data for 4i. White powder; yield, 79.1\%, m.p. 158-160 ${ }^{\circ} \mathrm{C} ;{ }^{1} \mathrm{H}$ NMR $(600 \mathrm{MHz}$, DMSO- $\left.d_{6}\right): \delta 9.90(\mathrm{t}, J=5.8 \mathrm{~Hz}, 1 \mathrm{H}, \mathrm{CONH}), 8.03(\mathrm{~d}, J=4.7 \mathrm{~Hz}, 1 \mathrm{H}, \mathrm{H}-4$, thiophene), 7.93 (d, $J=3.1 \mathrm{~Hz}, 1 \mathrm{H}, \mathrm{H}-2$, thiophene), 7.63-7.60 (m, 2H, H-16, 19, phenyl), 7.36-7.33 (m, 2H, H-3, thiophene, H-15, phenyl), 4.49 (d, $J=5.9 \mathrm{~Hz}, 2 \mathrm{H}$, $\left.\mathrm{NHCH}_{2}\right) \cdot{ }^{13} \mathrm{C}$ NMR $\left(150 \mathrm{MHz}, \mathrm{DMSO}-d_{6}\right): \delta 161.36(\mathrm{C}=\mathrm{O}), 157.74,153.16,139.56$, 132.67, 131.40, 130.91, 130.47, 129.60, 129.42, 128.94, 127.80, 123.60, 41.50. ESI-HRMS: $m / z$ calcd. for $\mathrm{C}_{14} \mathrm{H}_{10} \mathrm{Cl}_{2} \mathrm{~N}_{3} \mathrm{O}_{2} \mathrm{~S}[\mathrm{M}+\mathrm{H}]^{+}: 353.9871$; found 353.9873 .

Data for 4j. White powder; yield, 49.3\%, m.p. 157-159 ${ }^{\circ} \mathrm{C} ;{ }^{1} \mathrm{H}$ NMR $(600 \mathrm{MHz}$, DMSO- $\left.d_{6}\right): \delta 9.93(\mathrm{t}, J=6.1 \mathrm{~Hz}, 1 \mathrm{H}, \mathrm{CONH}), 8.03\left(\mathrm{dd}, J_{1}=5.0 \mathrm{~Hz}, J_{2}=1.1 \mathrm{~Hz}, 1 \mathrm{H}\right.$, $\mathrm{H}-4$, thiophene), 7.93 (dd, $J_{1}=3.7 \mathrm{~Hz}, J_{2}=1.1 \mathrm{~Hz}, 1 \mathrm{H}, \mathrm{H}-2$, thiophene), 7.49 (d, $J=$ 8.7 Hz, 2H, H-15, 19, phenyl), 7.35-7.32 (m, 3H, H-3, thiophene, H-16, 18, phenyl), $4.52\left(\mathrm{~d}, J=6.2 \mathrm{~Hz}, 2 \mathrm{H}, \mathrm{NHCH}_{2}\right) .{ }^{13} \mathrm{C} \mathrm{NMR}\left(150 \mathrm{MHz}, \mathrm{DMSO}-d_{6}\right): \delta 161.88(\mathrm{C}=\mathrm{O})$, 158.28, 153.56, $147.86\left(\mathrm{~d},{ }^{2} J_{\mathrm{C}-\mathrm{F}}=1.1 \mathrm{~Hz}, \mathrm{C}-17\right), 138.34,133.10,131.85,129.84$, 129.36, 124.08, 121.41, 120.61 (q, $\left.{ }^{1} J_{\mathrm{C}-\mathrm{F}}=272.2 \mathrm{~Hz}, \mathrm{OCF}_{3}\right), 42.37$. ESI-HRMS: $m / z$ calcd. for $\mathrm{C}_{15} \mathrm{H}_{11} \mathrm{~F}_{3} \mathrm{~N}_{3} \mathrm{O}_{3} \mathrm{~S}[\mathrm{M}+\mathrm{H}]^{+}:$370.0473; found 370.0469 .

Data for 4k. White powder; yield, 65.3\%, m.p. 199-201 ${ }^{\circ} \mathrm{C} ;{ }^{1} \mathrm{H}$ NMR $(600 \mathrm{MHz}$, DMSO- $\left.d_{6}\right): \delta 9.90(\mathrm{t}, J=5.9 \mathrm{~Hz}, 1 \mathrm{H}, \mathrm{CONH}), 8.04\left(\mathrm{dd}, J_{1}=5.0 \mathrm{~Hz}, J_{2}=1.0 \mathrm{~Hz}, 1 \mathrm{H}\right.$, $\mathrm{H}-4$, thiophene), $7.94\left(\mathrm{dd}, J_{1}=3.7 \mathrm{~Hz}, J_{2}=1.0 \mathrm{~Hz}, 1 \mathrm{H}, \mathrm{H}-2\right.$, thiophene), 7.64 (d, $J=$ $1.9 \mathrm{~Hz}, 1 \mathrm{H}, \mathrm{H}-18$, phenyl), 7.47-7.42 (m, 2H, H-15, 16, phenyl), 7.34 (dd, $J_{1}=4.9 \mathrm{~Hz}$, $J_{2}=3.8 \mathrm{~Hz}, 1 \mathrm{H}, \mathrm{H}-3$, thiophene), $4.53\left(\mathrm{~d}, J=5.9 \mathrm{~Hz}, 2 \mathrm{H}, \mathrm{NHCH}_{2}\right) .{ }^{13} \mathrm{C}$ NMR $(150$ MHz, DMSO- $\left.d_{6}\right): \delta 161.44(\mathrm{C}=\mathrm{O}), 157.70,153.29,134.36,132.87,132.79,132.52$, 131.53, 130.27, 129.03, 128.64, 127.36, 123.57, 40.12. ESI-HRMS: $m / z$ calcd. for $\mathrm{C}_{14} \mathrm{H}_{10} \mathrm{Cl}_{2} \mathrm{~N}_{3} \mathrm{O}_{2} \mathrm{~S}[\mathrm{M}+\mathrm{H}]^{+}: 353.9871$; found 353.9867 .

Data for 41. Yellow solid; yield, 34.3\%, m.p. 203-205 ${ }^{\circ} \mathrm{C}$; ${ }^{1} \mathrm{H}$ NMR (600 MHz, DMSO- $\left.d_{6}\right): \delta 9.46(\mathrm{t}, J=5.6 \mathrm{~Hz}, 1 \mathrm{H}, \mathrm{CONH}), 8.03(\mathrm{~d}, J=4.9 \mathrm{~Hz}, 1 \mathrm{H}, \mathrm{H}-4$, thiophene), 7.93 (d, $J=3.6 \mathrm{~Hz}, 1 \mathrm{H}, \mathrm{H}-2$, thiophene), 7.66 (d, $J=7.9 \mathrm{~Hz}, 2 \mathrm{H}, \mathrm{H}-16$, 20, phenyl), 7.50 (d, $J=8.0 \mathrm{~Hz}, 2 \mathrm{H}, \mathrm{H}-17,19$, phenyl), 7.34 (t, $J=4.0 \mathrm{~Hz}, 1 \mathrm{H}, \mathrm{H}-3$, 
thiophene), 3.58 (q, $J=6.8 \mathrm{~Hz}, 2 \mathrm{H}, \mathrm{NHCH}_{2} \mathrm{CH}_{2}$ ), 2.99 (t, $J=7.1 \mathrm{~Hz}, 2 \mathrm{H}$, $\left.\mathrm{NHCH}_{2} \underline{\mathrm{CH}_{2}}\right) .{ }^{13} \mathrm{C}$ NMR $\left(150 \mathrm{MHz}, \mathrm{DMSO}-d_{6}\right): \delta 161.86(\mathrm{C}=\mathrm{O}), 158.26,153.36$, 144.51, 133.20, 131.91, 130.07, 129.46, 127.50 (q, $\left.{ }^{2} J_{\mathrm{C}-\mathrm{F}}=3.7 \mathrm{~Hz}, \mathrm{C}-18\right), 124.91$ (q, $\left.{ }^{1} J_{\mathrm{C}-\mathrm{F}}=270.4 \mathrm{~Hz}, \mathrm{CF}_{3}\right), 40.66,34.74$. ESI-HRMS: $m / z$ calcd. for $\mathrm{C}_{16} \mathrm{H}_{13} \mathrm{~N}_{3} \mathrm{O}_{2} \mathrm{~F}_{3} \mathrm{~S}[\mathrm{M}+$ $\mathrm{H}]^{+}: 368.0681$; found 368.0685 .

Data for 4m. White solid; yield, 51.4\%, m.p. 145-147 ${ }^{\circ} \mathrm{C} ;{ }^{1} \mathrm{H}$ NMR (600 MHz, DMSO- $\left.d_{6}\right): \delta 9.81(\mathrm{t}, J=5.2 \mathrm{~Hz}, 1 \mathrm{H}, \mathrm{CONH}), 8.02(\mathrm{~d}, J=4.9 \mathrm{~Hz}, 1 \mathrm{H}, \mathrm{H}-4$, thiophene), 7.92 (d, $J=3.6 \mathrm{~Hz}, 1 \mathrm{H}, \mathrm{H}-2$, thiophene), $7.45-7.39$ (m, 1H, H-17), 7.33 (t, $J=4.5 \mathrm{~Hz}, 1 \mathrm{H}, \mathrm{H}-3$, thiophene), 7.11 (t, $J=7.9 \mathrm{~Hz}, 2 \mathrm{H}, \mathrm{H}-16,18$, phenyl), 4.55 (d, $J$ $\left.=5.3 \mathrm{~Hz}, 2 \mathrm{H}, \mathrm{NHCH}_{2}\right) \cdot{ }^{13} \mathrm{C} \mathrm{NMR}\left(150 \mathrm{MHz}, \mathrm{DMSO}-d_{6}\right): \delta 161.91(\mathrm{C}=\mathrm{O}), 161.71(\mathrm{dd}$, $\left.{ }^{1} J_{\mathrm{C}-\mathrm{F}}=247.1 \mathrm{~Hz},{ }^{3} J_{\mathrm{C}-\mathrm{F}}=8.2 \mathrm{~Hz}\right), 158.15,153.29,133.29,132.06,130.73\left(\mathrm{t},{ }^{3} J_{\mathrm{C}-\mathrm{F}}=\right.$ $10.1 \mathrm{~Hz}), 129.52,124.14,113.72\left(\mathrm{t},{ }^{2} J_{\mathrm{C}-\mathrm{F}}=18.9 \mathrm{~Hz}\right), 112.07\left(\mathrm{dd},{ }^{2} J_{\mathrm{C}-\mathrm{F}}=20.3 \mathrm{~Hz}\right.$, $\left.{ }^{4} J_{\mathrm{C}-\mathrm{F}}=4.8 \mathrm{~Hz}\right), 31.77(\mathrm{t}, J=3.7 \mathrm{~Hz}, \mathrm{C}-14)$. ESI-HRMS: $m / z$ calcd. for $\mathrm{C}_{14} \mathrm{H}_{10} \mathrm{~F}_{2} \mathrm{~N}_{3} \mathrm{O}_{2} \mathrm{~S}: 322.0462[\mathrm{M}+\mathrm{H}]^{+}$; found 322.0465 .

Data for 4n. White solid; yield, 78.2\%, m.p. 153-155 ${ }^{\circ} \mathrm{C} ;{ }^{1} \mathrm{H}$ NMR $(600 \mathrm{MHz}$, DMSO-d $\left.)_{6}\right): \delta 9.88(\mathrm{t}, J=6.1 \mathrm{~Hz}, 1 \mathrm{H}, \mathrm{CONH}), 8.03(\mathrm{~d}, J=4.9 \mathrm{~Hz}, 1 \mathrm{H}, \mathrm{H}-4$, thiophene), $7.93(\mathrm{~d}, J=3.6 \mathrm{~Hz}, 1 \mathrm{H}, \mathrm{H}-2$, thiophene), 7.36-7.32 (m, 5H, $\mathrm{H}-15,16,17,18,19$, phenyl), 7.28-7.25 (m, 1H, H-3, thiophene), 4.49 (d, $J=6.2 \mathrm{~Hz}, 2 \mathrm{H}$, $\left.\mathrm{NHCH}_{2}\right) .{ }^{13} \mathrm{C}$ NMR (150 MHz, DMSO- $\left.d_{6}\right): \delta 161.34(\mathrm{C}=\mathrm{O}), 157.83,152.98,138.33$, 132.65, 131.40, 128.93, 128.31, 127.43, 127.02, 123.60, 42.53. ESI-HRMS: $m / z$ calcd. for $\mathrm{C}_{14} \mathrm{H}_{12} \mathrm{~N}_{3} \mathrm{O}_{2} \mathrm{~S}: 286.0650[\mathrm{M}+\mathrm{H}]^{+}$; found 286.0653 .

Data for 4o. White solid; yield, 73.3\%, m.p. 207-209 ${ }^{\circ} \mathrm{C}$; ${ }^{1} \mathrm{H}$ NMR (600 MHz, DMSO- $\left.d_{6}\right): \delta 9.81(\mathrm{t}, J=6.1 \mathrm{~Hz}, 1 \mathrm{H}, \mathrm{CONH}), 8.02(\mathrm{~d}, J=5.0 \mathrm{~Hz}, 1 \mathrm{H}, \mathrm{H}-4$, thiophene), 7.93-7.92 (m, 1H, H-2, thiophene), 7.34-7.32 (m, 1H, H-3, thiophene), 6.93 (s, 1H, H-15, phenyl), 6.87 (d, $J=7.9 \mathrm{~Hz}, 1 \mathrm{H}, \mathrm{H}-19$, phenyl), 6.83 (dd, $J_{1}=7.9 \mathrm{~Hz}, J_{2}=0.9$ $\mathrm{Hz}, 1 \mathrm{H}, \mathrm{H}-16$, phenyl), 5.99 (s, 2H, $\left.\mathrm{CH}_{2}\right), 4.39$ (d, $\left.J=6.2 \mathrm{~Hz}, 2 \mathrm{H}, \mathrm{NHCH}_{2}\right) .{ }^{13} \mathrm{C} \mathrm{NMR}$ (150 MHz, DMSO- $\left.d_{6}\right): \delta 161.32(\mathrm{C}=\mathrm{O}), 157.85,152.88,147.23,146.25,132.64$, 132.18, 131.39, 128.93, 123.62, 120.83, 108.17, 108.01, 100.85, 42.32. ESI-HRMS: $m / z$ calcd. For $\mathrm{C}_{15} \mathrm{H}_{12} \mathrm{~N}_{3} \mathrm{O}_{4} \mathrm{~S}: 330.0549[\mathrm{M}+\mathrm{H}]^{+}$; found 330.0544 . 
Data for 5a. Yellow solid; yield, 85.1\%, m.p. 136-138 ${ }^{\circ} \mathrm{C} ;{ }^{1} \mathrm{H}$ NMR (600 MHz, DMSO- $\left.d_{6}\right): \delta 9.91(\mathrm{t}, J=6.1 \mathrm{~Hz}, 1 \mathrm{H}, \mathrm{CONH}), 8.12$ (s, 1H, H-2, furan), 7.48 (d, $J=3.5$ Hz, 1H, H-4, furan), 7.41 (d, $J=8.5 \mathrm{~Hz}, 2 \mathrm{H}, \mathrm{H}-16,18$, phenyl), 7.37 (d, $J=8.5 \mathrm{~Hz}$, 2H, H-15, 19, phenyl), 6.84 (dd, $J_{1}=3.5 \mathrm{~Hz}, J_{2}=1.7 \mathrm{~Hz}, \mathrm{H}-3$, furan), 4.47 (d, $J=6.2$ $\left.\mathrm{Hz}, 2 \mathrm{H}, \mathrm{NHCH}_{2}\right) .{ }^{13} \mathrm{C} \mathrm{NMR}\left(150 \mathrm{MHz}, \mathrm{DMSO}-d_{6}\right): \delta 157.79(\mathrm{C}=\mathrm{O}), 157.56,152.98$, 147.67, 138.10, 137.36, 131.62, 129.33, 128.26, 115.92, 112.88, 41.90. ESI-HRMS: $m / z$ calcd. for $\mathrm{C}_{14} \mathrm{H}_{11} \mathrm{ClN}_{3} \mathrm{O}_{3}: 304.0489[\mathrm{M}+\mathrm{H}]^{+}$; found 304.0493 .

Data for 5b. White solid; yield, 84.4\%, m.p. 137-139 ${ }^{\circ} \mathrm{C}$; ${ }^{1} \mathrm{H}$ NMR (600 MHz, DMSO- $\left.d_{6}\right): \delta 9.90(\mathrm{t}, J=6.0 \mathrm{~Hz}, 1 \mathrm{H}, \mathrm{CONH}), 8.12$ (s, 1H, H-2, furan), 7.48 (d, $J=$ $3.5 \mathrm{~Hz}, 1 \mathrm{H}, \mathrm{H}-4$, furan), 7.39 (dd, $J_{1}=8.4 \mathrm{~Hz}, J_{2}=5.7 \mathrm{~Hz}, 2 \mathrm{H}, \mathrm{H}-15,19$, phenyl), 7.17 (t, $J=8.9 \mathrm{~Hz}, 2 \mathrm{H}, \mathrm{H}-16,18$, phenyl), 6.84 (t, $J_{1}=3.8 \mathrm{~Hz}, J_{2}=1.7 \mathrm{~Hz}, 1 \mathrm{H}, \mathrm{H}-3$, furan), $4.46\left(\mathrm{~d}, J=6.2 \mathrm{~Hz}, 2 \mathrm{H}, \mathrm{NHCH}_{2}\right) \cdot{ }^{13} \mathrm{C} \mathrm{NMR}(150 \mathrm{MHz}, \mathrm{DMSO}): \delta 161.80\left(\mathrm{~d},{ }^{1} J_{\mathrm{C}-\mathrm{F}}=\right.$ $241.0 \mathrm{~Hz}, \mathrm{C}-17), 158.28,158.09,153.41,148.15,138.61,135.04\left(\mathrm{~d},{ }^{4} J_{\mathrm{C}-\mathrm{F}}=2.9 \mathrm{~Hz}\right.$, C-14), $130.02\left(\mathrm{~d},{ }^{3} J_{\mathrm{C}-\mathrm{F}}=8.1 \mathrm{~Hz}, \mathrm{C}-15,19\right), 116.40,115.51\left(\mathrm{~d},{ }^{2} J_{\mathrm{C}-\mathrm{F}}=21.1 \mathrm{~Hz}, \mathrm{C}-16\right.$, 18), 113.37, 42.36. ESI-HRMS: $m / z$ calcd. for $\mathrm{C}_{14} \mathrm{H}_{11} \mathrm{FN}_{3} \mathrm{O}_{3}: 288.0784[\mathrm{M}+\mathrm{H}]^{+}$; found 288.0781 .

Data for 5c. White solid; yield, 62.6\%, m.p. 133-135 ${ }^{\circ} \mathrm{C} ;{ }^{1} \mathrm{H}$ NMR $(600 \mathrm{MHz}$, DMSO- $\left.d_{6}\right): \delta 9.86(\mathrm{t}, J=6.2 \mathrm{~Hz}, 1 \mathrm{H}, \mathrm{CONH}), 8.13(\mathrm{~d}, J=1.1 \mathrm{~Hz}, 1 \mathrm{H}, \mathrm{H}-2$, furan), 7.48 (d, $J=3.4 \mathrm{~Hz}, 1 \mathrm{H}, \mathrm{H}-4$, furan), 7.36 (d, $J=8.3 \mathrm{~Hz}, 2 \mathrm{H}, \mathrm{H}-16,18$, phenyl), 7.28 (d, $J=8.3 \mathrm{~Hz}, 2 \mathrm{H}, \mathrm{H}-15,19$, phenyl), 6.84 (dd, $J_{1}=3.6 \mathrm{~Hz}, J_{2}=1.7 \mathrm{~Hz}, 1 \mathrm{H}, \mathrm{H}-3$, furan), $4.44\left(\mathrm{~d}, J=6.2 \mathrm{~Hz}, 2 \mathrm{H}, \mathrm{NHCH}_{2}\right), 1.27\left(\mathrm{~s}, 9 \mathrm{H}, \mathrm{C}\left(\mathrm{CH}_{3}\right)_{3}\right) .{ }^{13} \mathrm{C} \mathrm{NMR}(150 \mathrm{MHz}$, DMSO): $\delta 157.75(\mathrm{C}=\mathrm{O}), 157.63,152.81,149.45,147.64,138.10,135.33,127.31$, 125.04, 115.89, 112.86, 42.24, 34.14, 31.11. ESI-HRMS: $m / z$ calcd. for $\mathrm{C}_{18} \mathrm{H}_{20} \mathrm{~N}_{3} \mathrm{O}_{3}$ : $326.1505[\mathrm{M}+\mathrm{H}]^{+}$; found 326.1501 .

Data for 5d. White solid; yield, 85.6 \%, m.p. 150-151 ${ }^{\circ} \mathrm{C} ;{ }^{1} \mathrm{H}$ NMR $(600 \mathrm{MHz}$, DMSO- $\left.d_{6}\right): \delta 9.85(\mathrm{t}, J=6.1 \mathrm{~Hz}, 1 \mathrm{H}, \mathrm{CONH}), 8.12$ (s, 1H, H-2, furan), 7.47 (d, $J=$ $3.5 \mathrm{~Hz}, 1 \mathrm{H}, \mathrm{H}-4$, furan), 7.23 (d, $J=7.8 \mathrm{~Hz}, 2 \mathrm{H}, \mathrm{H}-15$,19, phenyl), 7.14 (d, $J=7.8$ Hz, 2H, H-16, 18, phenyl), 6.84 (dd, $J_{1}=3.0 \mathrm{~Hz}, J_{2}=1.2 \mathrm{~Hz}, 1 \mathrm{H}, \mathrm{H}-3$, furan), 4.43 (d, $\left.J=6.2 \mathrm{~Hz}, 2 \mathrm{H}, \mathrm{NHCH}_{2}\right), 2.28\left(\mathrm{~s}, 3 \mathrm{H}, \mathrm{CH}_{3}\right) \cdot{ }^{13} \mathrm{C} \mathrm{NMR}\left(150 \mathrm{MHz}, \mathrm{DMSO}-d_{6}\right): \delta 157.76$ 
$(\mathrm{C}=\mathrm{O}), 157.63,152.83,147.64,138.11,136.12,135.30,128.84,127.44,115.89$, 112.86, 42.29, 20.64. ESI-HRMS: $m / z$ calcd. for $\mathrm{C}_{15} \mathrm{H}_{14} \mathrm{~N}_{3} \mathrm{O}_{3}[\mathrm{M}+\mathrm{H}]^{+}$: 284.1035; found 284.1039.

Data for 5e. White solid; yield, 56.2\%, m.p. 144-146 ${ }^{\circ} \mathrm{C} ;{ }^{1} \mathrm{H}$ NMR $(600 \mathrm{MHz}$, DMSO-d $\left.d_{6}\right): \delta 9.91(\mathrm{t}, J=6.1 \mathrm{~Hz}, 1 \mathrm{H}, \mathrm{CONH}), 8.13$ (t, $J=0.8 \mathrm{~Hz}, 1 \mathrm{H}, \mathrm{H}-2$, furan), 7.48 (d, $J=3.5 \mathrm{~Hz}, 1 \mathrm{H}, \mathrm{H}-4$, furan), 7.39 (t, $J=7.9 \mathrm{~Hz}, 1 \mathrm{H}, \mathrm{H}-16$, phenyl), 7.18 (t, $J$ $=7.1 \mathrm{~Hz}, 2 \mathrm{H}, \mathrm{H}-17,19$, phenyl), 7.11-7.08 (m, 1H, H-15, phenyl), 6.85 (dd, $J_{1}=3.5$ $\mathrm{Hz}, J_{2}=1.7 \mathrm{~Hz}, 1 \mathrm{H}, \mathrm{H}-3$, furan), $4.50\left(\mathrm{~d}, J=6.2 \mathrm{~Hz}, 2 \mathrm{H}, \mathrm{NHCH}_{2}\right) .{ }^{13} \mathrm{C} \mathrm{NMR}(150$ MHz, DMSO- $\left.d_{6}\right): \delta 162.80\left(\mathrm{~d},{ }^{1} J_{\mathrm{C}-\mathrm{F}}=242.0 \mathrm{~Hz}, \mathrm{C}-18\right), 158.39(\mathrm{C}=\mathrm{O}), 158.18,153.66$, $148.22,141.87\left(\mathrm{~d},{ }^{3} J_{\mathrm{C}-\mathrm{F}}=7.2 \mathrm{~Hz}, \mathrm{C}-14\right), 138.73,130.86\left(\mathrm{~d},{ }^{3} J_{\mathrm{C}-\mathrm{F}}=8.2 \mathrm{~Hz}, \mathrm{C}-16\right)$, $123.97\left(\mathrm{~d},{ }^{4} J_{\mathrm{C}-\mathrm{F}}=2.6 \mathrm{~Hz}, \mathrm{C}-15\right), 116.50,114.76,114.69\left(\mathrm{~d},{ }^{2} J_{\mathrm{C}-\mathrm{F}}=21.7 \mathrm{~Hz}, \mathrm{C}-17\right)$, $114.38\left(\mathrm{~d},{ }^{2} J_{\mathrm{C}-\mathrm{F}}=20.8 \mathrm{~Hz}, \mathrm{C}-19\right), 113.47$, 42.65. ESI-HRMS: $m / z$ calcd. for $\mathrm{C}_{14} \mathrm{H}_{11} \mathrm{FN}_{3} \mathrm{O}_{3}[\mathrm{M}+\mathrm{H}]^{+}$: 288.0784; found 288.0781.

Data for $5 f$. White solid; yield, 84.5\%, m.p. $147-149{ }^{\circ} \mathrm{C} ;{ }^{1} \mathrm{H}$ NMR $(600 \mathrm{MHz}$, DMSO- $\left.d_{6}\right): \delta 9.89$ (t, $\left.J=5.8 \mathrm{~Hz}, 1 \mathrm{H}, \mathrm{CONH}\right), 8.13$ (s, 1H, H-2, furan), 7.48 (d, $J=$ $3.5 \mathrm{~Hz}, 1 \mathrm{H}, \mathrm{H}-4$, furan), 7.42 (t, $J=7.6 \mathrm{~Hz}, 1 \mathrm{H}, \mathrm{H}-15$, phenyl), 7.34 (dd, $J_{1}=13.7 \mathrm{~Hz}$, $J_{2}=7.0 \mathrm{~Hz}, 1 \mathrm{H}, \mathrm{H}-17$, phenyl), 7.21-7.17 (m, 2H, H-16,18, phenyl), 6.84 (t, $J=1.7$ $\mathrm{Hz}, 1 \mathrm{H}, \mathrm{H}-3$, furan), 4.54 (d, $\left.J=5.9 \mathrm{~Hz}, 2 \mathrm{H}, \mathrm{NHCH}_{2}\right) .{ }^{13} \mathrm{C} \mathrm{NMR}(150 \mathrm{MHz}$, DMSO- $\left.d_{6}\right): \delta 160.42\left(\mathrm{~d},{ }^{1} J_{\mathrm{C}-\mathrm{F}}=243.2 \mathrm{~Hz}, \mathrm{C}-19\right), 158.28(\mathrm{C}=\mathrm{O}), 157.99,153.53$, 148.16, 138.59, $130.12\left(\mathrm{~d},{ }^{3} J_{\mathrm{C}-\mathrm{F}}=4.1 \mathrm{~Hz}, \mathrm{C}-15\right), 129.66\left(\mathrm{~d},{ }^{3} J_{\mathrm{C}-\mathrm{F}}=7.9 \mathrm{~Hz}, \mathrm{C}-17\right)$, $125.34\left(\mathrm{~d},{ }^{2} J_{\mathrm{C}-\mathrm{F}}=14.5 \mathrm{~Hz}, \mathrm{C}-14\right), 124.82\left(\mathrm{~d},{ }^{4} J_{\mathrm{C}-\mathrm{F}}=3.4 \mathrm{~Hz}, \mathrm{C}-16\right), 116.43,115.59(\mathrm{~d}$, $\left.{ }^{2} J_{\mathrm{C}-\mathrm{F}}=20.9 \mathrm{~Hz}, \mathrm{C}-18\right), 113.37,36.88(\mathrm{~d}, J=4.7 \mathrm{~Hz}, \mathrm{C}-13)$. ESI-HRMS: $m / z$ calcd.for $\mathrm{C}_{14} \mathrm{H}_{11} \mathrm{FN}_{3} \mathrm{O}_{3}[\mathrm{M}+\mathrm{H}]^{+}$: 288.0784; found 288.0789.

Data for 5g. White solid; yield, 46.0\%, m.p. 184-186 ${ }^{\circ} \mathrm{C} ;{ }^{1} \mathrm{H}$ NMR $(600 \mathrm{MHz}$, DMSO-d $\left.d_{6}\right): \delta 9.99$ (t, $\left.J=6.4 \mathrm{~Hz}, 1 \mathrm{H}, \mathrm{CONH}\right), 8.13$ (s, 1H, H-2, furan), 7.71 (d, $J=$ $8.0 \mathrm{~Hz}, 2 \mathrm{H}, \mathrm{H}-15,19$, phenyl), 7.58 (d, $J=7.9 \mathrm{~Hz}, 2 \mathrm{H}, \mathrm{H}-16,18$, phenyl), 7.48 (d, $J=$ $3.5 \mathrm{~Hz}, 1 \mathrm{H}, \mathrm{H}-4$, furan), 6.84 (t, $J=1.7 \mathrm{~Hz} 1 \mathrm{H}, \mathrm{H}-3$, furan), 4.58 (d, $J=5.9 \mathrm{~Hz}, 2 \mathrm{H}$, $\left.\mathrm{NHCH}_{2}\right) \cdot{ }^{13} \mathrm{C}$ NMR (150 MHz, DMSO-d $)_{6}: \delta 158.39(\mathrm{C}=\mathrm{O}), 158.10,153.67,148.24$, $143.71,138.66,128.66,128.29\left(\mathrm{q},{ }^{2} J_{\mathrm{C}-\mathrm{F}}=31.5 \mathrm{~Hz}, \mathrm{C}-17\right), 125.76\left(\mathrm{q},{ }^{3} J_{\mathrm{C}-\mathrm{F}}=3.8 \mathrm{~Hz}\right.$, 
C-16,18), 124.89 (q, ${ }^{1} J_{\mathrm{C}-\mathrm{F}}=284.8 \mathrm{~Hz}, \mathrm{CF}_{3}$ ), 116.51, 113.44. 42.78. ESI-HRMS: $m / z$ calcd.for $\mathrm{C}_{15} \mathrm{H}_{11} \mathrm{~F}_{3} \mathrm{~N}_{3} \mathrm{O}_{3}[\mathrm{M}+\mathrm{H}]^{+}$: 338.0753; found 338.0750.

Data for 5h. White solid; yield, 84.5\%, m.p. 162-164 ${ }^{\circ} \mathrm{C}$; ${ }^{1} \mathrm{H}$ NMR (600 MHz, DMSO- $\left.d_{6}\right): \delta 9.43(\mathrm{t}, J=5.6 \mathrm{~Hz}, 1 \mathrm{H}, \mathrm{CONH}), 8.13$ (s, 1H, H-2, furan), 7.48 (d, $J=$ $3.5 \mathrm{~Hz}, 1 \mathrm{H}, \mathrm{H}-4$, furan), 7.29 (dd, $J_{1}=8.1 \mathrm{~Hz}, J_{2}=5.8 \mathrm{~Hz}, 2 \mathrm{H}, \mathrm{H}-16,20$, phenyl), 7.12 (t, $J=8.8 \mathrm{~Hz}, 2 \mathrm{H}, \mathrm{H}-17,19$, phenyl), 6.84 (t, $J=1.8 \mathrm{~Hz}, 1 \mathrm{H}, \mathrm{H}-3$, furan), 3.52 (q, $\left.J=6.8 \mathrm{~Hz}, 2 \mathrm{H}, \mathrm{NHCH}_{2} \mathrm{CH}_{2}\right), 2.87$ (t, $\left.J=7.3 \mathrm{~Hz}, 2 \mathrm{H}, \mathrm{NHCH}_{2} \underline{\mathrm{CH}}_{2}\right) \cdot{ }^{13} \mathrm{C}$ NMR (150 MHz, DMSO- $\left.d_{6}\right): \delta 162.02\left(\mathrm{~d},{ }^{1} J_{\mathrm{C}-\mathrm{F}}=240.1 \mathrm{~Hz}, \mathrm{C}-18\right), 158.91(\mathrm{C}=\mathrm{O}), 158.70,153.85$, 148.80, 139.22, $136.23\left(\mathrm{~d},{ }^{4} J_{\mathrm{C}-\mathrm{F}}=3.0 \mathrm{~Hz}, \mathrm{C}-15\right), 131.59$ (d, $\left.{ }^{3} J_{\mathrm{C}-\mathrm{F}}=7.9 \mathrm{~Hz}, \mathrm{C}-16,20\right)$, $117.05,116.15\left(\mathrm{~d},{ }^{2} J_{\mathrm{C}-\mathrm{F}}=20.9 \mathrm{~Hz}, \mathrm{C}-17,19\right), 114.01,41.75,34.78$. ESI-HRMS: $m / z$ calcd.for $\mathrm{C}_{15} \mathrm{H}_{13} \mathrm{FN}_{3} \mathrm{O}_{3}[\mathrm{M}+\mathrm{H}]^{+}$: 302.0941; found 302.0937.

Data for 5i. White solid; yield, 64.5\%, m.p. 187-189 ${ }^{\circ} \mathrm{C} ;{ }^{1} \mathrm{H}$ NMR $(600 \mathrm{MHz}$, DMSO- $\left.d_{6}\right): \delta 9.92(\mathrm{t}, J=6.1 \mathrm{~Hz}, 1 \mathrm{H}, \mathrm{CONH}), 8.13(\mathrm{~s}, 1 \mathrm{H}, \mathrm{H}-2$, furan), 7.63-7.61 (m, 2H, H-16, 19, phenyl), 7.48 (d, $J=3.5 \mathrm{~Hz}, 1 \mathrm{H}, 4-\mathrm{H}$, furan), 7.36 (dd, $J_{1}=8.3 \mathrm{~Hz}, J_{2}=$ $1.7 \mathrm{~Hz}, 1 \mathrm{H}, \mathrm{H}-15$, phenyl), 6.85 (dd, $J_{1}=3.5 \mathrm{~Hz}, J_{2}=1.6 \mathrm{~Hz}, 1 \mathrm{H}, 3-\mathrm{H}$, furan), 4.49 (d, $\left.J=6.2 \mathrm{~Hz}, 2 \mathrm{H}, \mathrm{NHCH}_{2}\right) \cdot{ }^{13} \mathrm{C} \mathrm{NMR}\left(150 \mathrm{MHz}, \mathrm{DMSO}-d_{6}\right): \delta 157.81(\mathrm{C}=\mathrm{O}), 157.54$, $153.13,147.69$, 139.57, 138.12, 130.93, 130.51, 129.62, 129.44, 127.83, 115.95, 112.91, 41.53. ESI-HRMS: $m / z$ calcd.for $\mathrm{C}_{14} \mathrm{H}_{10} \mathrm{Cl}_{2} \mathrm{~N}_{3} \mathrm{O}_{3}[\mathrm{M}+\mathrm{H}]^{+}: 338.0099$; found 338.0103 .

Data for 5j. White solid; yield, 43.9\%, m.p. 155-157 ${ }^{\circ} \mathrm{C} ;{ }^{1} \mathrm{H}$ NMR $(600 \mathrm{MHz}$, DMSO- $\left.d_{6}\right) \delta 9.94(\mathrm{t}, J=6.1 \mathrm{~Hz}, 1 \mathrm{H}, \mathrm{CONH}), 8.12$ (d, $J=1.1 \mathrm{~Hz}, 1 \mathrm{H}, \mathrm{H}-2$, furan), 7.49-7.47 (m, 3H, H-4, furan, H-15,19, phenyl), 7.34 (d, $J=8.2 \mathrm{~Hz}, 2 \mathrm{H}, \mathrm{H}-16,18$, phenyl), 6.84 (dd, $J_{1}=3.5 \mathrm{~Hz}, J_{2}=1.7 \mathrm{~Hz}, 1 \mathrm{H}, \mathrm{H}-3$, furan), 4.51 (d, $J=6.2 \mathrm{~Hz}, 2 \mathrm{H}$, $\left.\mathrm{NHCH}_{2}\right) \cdot{ }^{13} \mathrm{C}$ NMR $\left(150 \mathrm{MHz}, \mathrm{DMSO}-d_{6}\right) \delta 158.60(\mathrm{C}=\mathrm{O}), 158.36,153.80,148.45$, $148.15,138.90,138.65,130.14,120.86\left(\mathrm{q},{ }^{1} J_{\mathrm{C}-\mathrm{F}}=254.6 \mathrm{~Hz}, \mathrm{OCF}_{3}\right), 116.70,113.65$, 42.68. ESI-HRMS: $m / z$ calcd.for $\mathrm{C}_{15} \mathrm{H}_{11} \mathrm{~F}_{3} \mathrm{~N}_{3} \mathrm{O}_{4}[\mathrm{M}+\mathrm{H}]^{+}:$354.0702; found 354.0707.

Data for 5k. White solid; yield, 70.1\%, m.p. 206-208 ${ }^{\circ} \mathrm{C}$; ${ }^{1} \mathrm{H}$ NMR (600 MHz, DMSO- $\left.d_{6}\right): \delta 9.91(\mathrm{t}, J=5.1 \mathrm{~Hz}, 1 \mathrm{H}, \mathrm{CONH}), 8.13$ (s, 1H, H-2, furan), 7.64 (d, $J=0.9$ Hz, 1H, H-18, phenyl), 7.49 (d, $J=3.5 \mathrm{~Hz}, 1 \mathrm{H}, \mathrm{H}-4$, furan), $7.46-7.42$ (m, 2H, 
H-15,16, phenyl), 6.85 (t, $J=0.9 \mathrm{~Hz}, 1 \mathrm{H}, \mathrm{H}-3$, furan), 4.53 (d, $J=5.4 \mathrm{~Hz}, 2 \mathrm{H}$, $\left.\mathrm{NHCH}_{2}\right) \cdot{ }^{13} \mathrm{C}$ NMR $\left(150 \mathrm{MHz}, \mathrm{DMSO}-d_{6}\right): \delta 157.81(\mathrm{C}=\mathrm{O}), 157.45,153.19,147.72$, $138.07,134.31,132.83,132.47,130.21,128.60,127.32,115.99,112.90,40.08$. ESI-HRMS: $m / z$ calcd.for $\mathrm{C}_{14} \mathrm{H}_{10} \mathrm{Cl}_{2} \mathrm{~N}_{3} \mathrm{O}_{3}[\mathrm{M}+\mathrm{H}]^{+}$: 338.0099; found 338.0095.

Data for 5l. White solid; yield, 63.5\%, m.p. 211-213 ${ }^{\circ} \mathrm{C} ;{ }^{1} \mathrm{H}$ NMR (600 MHz, DMSO- $\left.d_{6}\right): \delta 9.46(\mathrm{t}, J=5.6 \mathrm{~Hz}, 1 \mathrm{H}, \mathrm{CONH}), 8.12(\mathrm{~s}, 1 \mathrm{H}, \mathrm{H}-2$, furan), 7.66 (d, $J=7.9$ Hz, 2H, H-16, 20, phenyl), 7.49-7.47 (m, 3H, 4-H, furan, H-17, 19, phenyl), 6.84 (t, $J$ $=7.9 \mathrm{~Hz}, 1 \mathrm{H}, \mathrm{H}-3$, furan), 3.57 (q, $\left.J=6.8 \mathrm{~Hz}, 2 \mathrm{H}, \mathrm{NHCH}_{2} \mathrm{CH}_{2}\right), 2.98(\mathrm{t}, J=7.1 \mathrm{~Hz}$, $\left.2 \mathrm{H}, \mathrm{NHCH}_{2} \underline{\mathrm{CH}}_{2}\right) .{ }^{13} \mathrm{C}$ NMR (150 MHz, DMSO- $\left.d_{6}\right): \delta 158.25(\mathrm{C}=\mathrm{O}), 153.27,148.15$, 144.48, 138.57, 130.05, $127.48\left(\mathrm{q},{ }^{2} J_{\mathrm{C}-\mathrm{F}}=31.7 \mathrm{~Hz}, \mathrm{C}-18\right), 125.62\left(\mathrm{q},{ }^{3} J_{\mathrm{C}-\mathrm{F}}=3.5 \mathrm{~Hz}\right.$, C-17, 19), 124.89 (q, ${ }^{1} J_{\mathrm{C}-\mathrm{F}}=270.3 \mathrm{~Hz}, \mathrm{CF}_{3}$ ), 116.39, 113.36, 40.64. ESI-HRMS: $m / z$ calcd.for $\mathrm{C}_{16} \mathrm{H}_{13} \mathrm{~F}_{3} \mathrm{~N}_{3} \mathrm{O}_{3}[\mathrm{M}+\mathrm{H}]^{+}$: 352.0909; found 352.0912.

Data for 5m. White solid; yield, 63.6\%, m.p. 160-162 ${ }^{\circ} \mathrm{C} ;{ }^{1} \mathrm{H}$ NMR (400 MHz, DMSO- $\left.d_{6}\right): \delta 9.85$ (t, $\left.J=5.4 \mathrm{~Hz}, 1 \mathrm{H}, \mathrm{CONH}\right), 8.15-8.14$ (m, 1H, H-2, furan), 7.49 (d, $J=3.5 \mathrm{~Hz}, 1 \mathrm{H}, \mathrm{H}-4$, furan), 7.47-7.41 (m, 1H, H-17), 7.13 (t, $J=8.0 \mathrm{~Hz}, 2 \mathrm{H}, \mathrm{H}-16$, 18), $6.86\left(\mathrm{dd}, J_{1}=3.6 \mathrm{~Hz}, J_{2}=1.8 \mathrm{~Hz}, 1 \mathrm{H}, \mathrm{H}-3\right.$, furan), $4.58(\mathrm{~d}, J=5.4 \mathrm{~Hz}$, $\left.2 \mathrm{H}, \mathrm{NHCH}_{2}\right) \cdot{ }^{13} \mathrm{C}$ NMR $\left(150 \mathrm{MHz}, \mathrm{DMSO}-d_{6}\right): \delta 161.68\left(\mathrm{~d},{ }^{1} J_{\mathrm{C}-\mathrm{F}}=246.9 \mathrm{~Hz},{ }^{3} J_{\mathrm{C}-\mathrm{F}}=\right.$ $7.9 \mathrm{~Hz}), 158.30,157.90,153.20,148.22,138.60,130.70\left(\mathrm{t},{ }^{3} J_{\mathrm{C}-\mathrm{F}}=10.4 \mathrm{~Hz}\right), 116.50$, $113.66\left(\mathrm{t},{ }^{2} J_{\mathrm{C}-\mathrm{F}}=18.9 \mathrm{~Hz}\right), 112.02\left(\mathrm{dd},{ }^{2} J_{\mathrm{C}-\mathrm{F}}=20.5 \mathrm{~Hz},{ }^{4} J_{\mathrm{C}-\mathrm{F}}=4.8 \mathrm{~Hz}\right), 31.77(\mathrm{~d}, J=$ $3.7 \mathrm{~Hz}, \mathrm{C}-13)$. ESI-HRMS: $m / z$ calcd.for $\mathrm{C}_{14} \mathrm{H}_{10} \mathrm{~F}_{2} \mathrm{~N}_{3} \mathrm{O}_{3}[\mathrm{M}+\mathrm{H}]^{+}:$306.0690; found 306.0695 .

Data for 5n. White solid; yield, 43.2\%, m.p. 148-150 ${ }^{\circ} \mathrm{C} ;{ }^{1} \mathrm{H}$ NMR (600 MHz, DMSO- $\left.d_{6}\right): \delta 9.89(\mathrm{t}, J=6.1 \mathrm{~Hz}, 1 \mathrm{H}, \mathrm{CONH}), 8.12$ (s, 1H, H-2, furan), 7.48 (d, $J=$ $3.5 \mathrm{~Hz}, 1 \mathrm{H}, \mathrm{H}-4$, furan), 7.35-7.33 (m, 4H, H-15,16,18,19), 7.28-7.25 (m, 1H, H-17), $6.84\left(\mathrm{dd}, J_{1}=3.4 \mathrm{~Hz}, J_{2}=1.6 \mathrm{~Hz}, 1 \mathrm{H}, \mathrm{H}-3\right.$, furan), 4.48 (d, $\left.J=6.2 \mathrm{~Hz}, 2 \mathrm{H}, \mathrm{NHCH}_{2}\right)$. ${ }^{13} \mathrm{C}$ NMR (150 MHz, DMSO-d $\left.d_{6}\right): \delta 157.77(\mathrm{C}=\mathrm{O}), 157.62,152.91,147.65,138.32$, 138.11, 128.31, 127.42, 127.02, 115.90, 112.87, 42.54. ESI-HRMS: $m / z$ calcd. for $\mathrm{C}_{14} \mathrm{H}_{12} \mathrm{~N}_{3} \mathrm{O}_{3}[\mathrm{M}+\mathrm{H}]^{+}:$270.0879; found 270.0880. 
Data for 5o. White solid; yield 55.0\%, m.p. 175-177 ${ }^{\circ} \mathrm{C} ;{ }^{1} \mathrm{H}$ NMR (600 MHz, DMSO- $\left.d_{6}\right): \delta 9.82(\mathrm{t}, J=6.2 \mathrm{~Hz}, 1 \mathrm{H}, \mathrm{CONH}), 8.13(\mathrm{~d}, J=1.1 \mathrm{~Hz}, 1 \mathrm{H}, \mathrm{H}-2$, furan), $7.48\left(\mathrm{dd}, J_{1}=3.6 \mathrm{~Hz}, J_{2}=0.5 \mathrm{~Hz}, 1 \mathrm{H}, \mathrm{H}-4\right.$, furan), $6.93(\mathrm{~d}, J=1.5 \mathrm{~Hz}, 1 \mathrm{H}, \mathrm{H}-15)$, $6.87(\mathrm{~d}, J=7.9 \mathrm{~Hz}, 1 \mathrm{H}, \mathrm{H}-19), 6.84\left(\mathrm{dd}, J_{1}=3.6 \mathrm{~Hz}, J_{2}=1.8 \mathrm{~Hz}, 1 \mathrm{H}, \mathrm{H}-3\right.$, furan), 6.83 $\left(\mathrm{dd}, J_{1}=7.9 \mathrm{~Hz}, J_{2}=1.6 \mathrm{~Hz}, 1 \mathrm{H}, \mathrm{H}-16\right), 5.99$ (s, 2H, $\left.\mathrm{CH}_{2}\right), 4.39$ (d, $J=6.2 \mathrm{~Hz}, 2 \mathrm{H}$, $\left.\mathrm{NHCH}_{2}\right) \cdot{ }^{13} \mathrm{C}$ NMR $\left(150 \mathrm{MHz}, \mathrm{DMSO}-d_{6}\right): \delta 157.75(\mathrm{C}=\mathrm{O}), 157.63,152.81,147.63$, $147.22,146.25,138.13,132.17,120.82,115.87,112.86,108.16,108.01,100.85$, 42.32. ESI-HRMS: $m / z$ calcd. for $\mathrm{C}_{15} \mathrm{H}_{12} \mathrm{~N}_{3} \mathrm{O}_{5}[\mathrm{M}+\mathrm{H}]^{+}: 314.0777$; found 314.0779. 
3. ${ }^{1} \mathrm{H}$ NMR, ${ }^{13} \mathrm{C}$ NMR spectra of compounds $\mathbf{3 a}, \mathbf{3 b}, \mathbf{4 a - 4 o}$ and $\mathbf{5 a - 5 o}$.
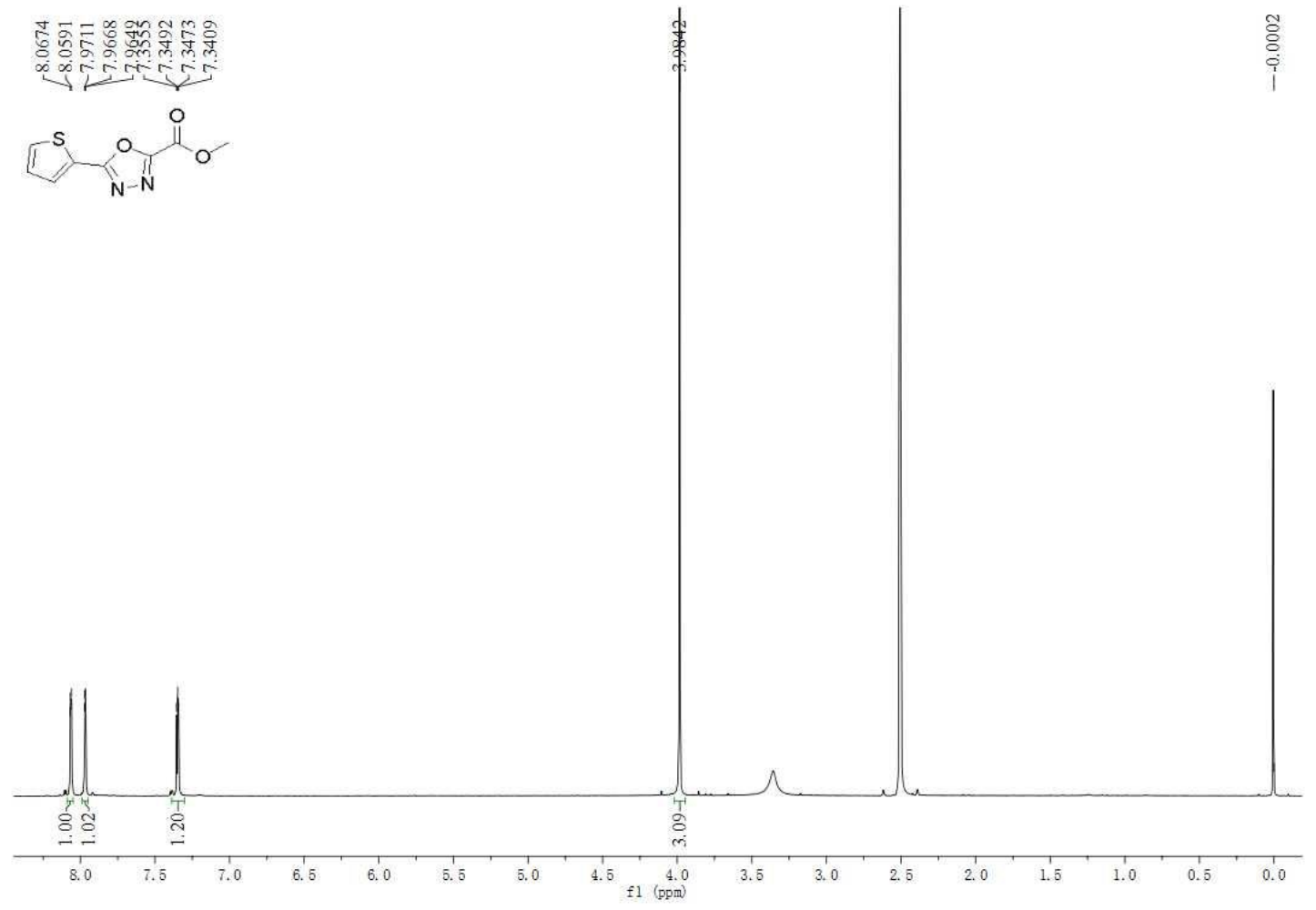

Figure S1. The ${ }^{1} \mathrm{H}$ NMR Spectrm $\left(600 \mathrm{MHz}, \mathrm{DMSO}-d_{6}\right)$ of intermediate 3a
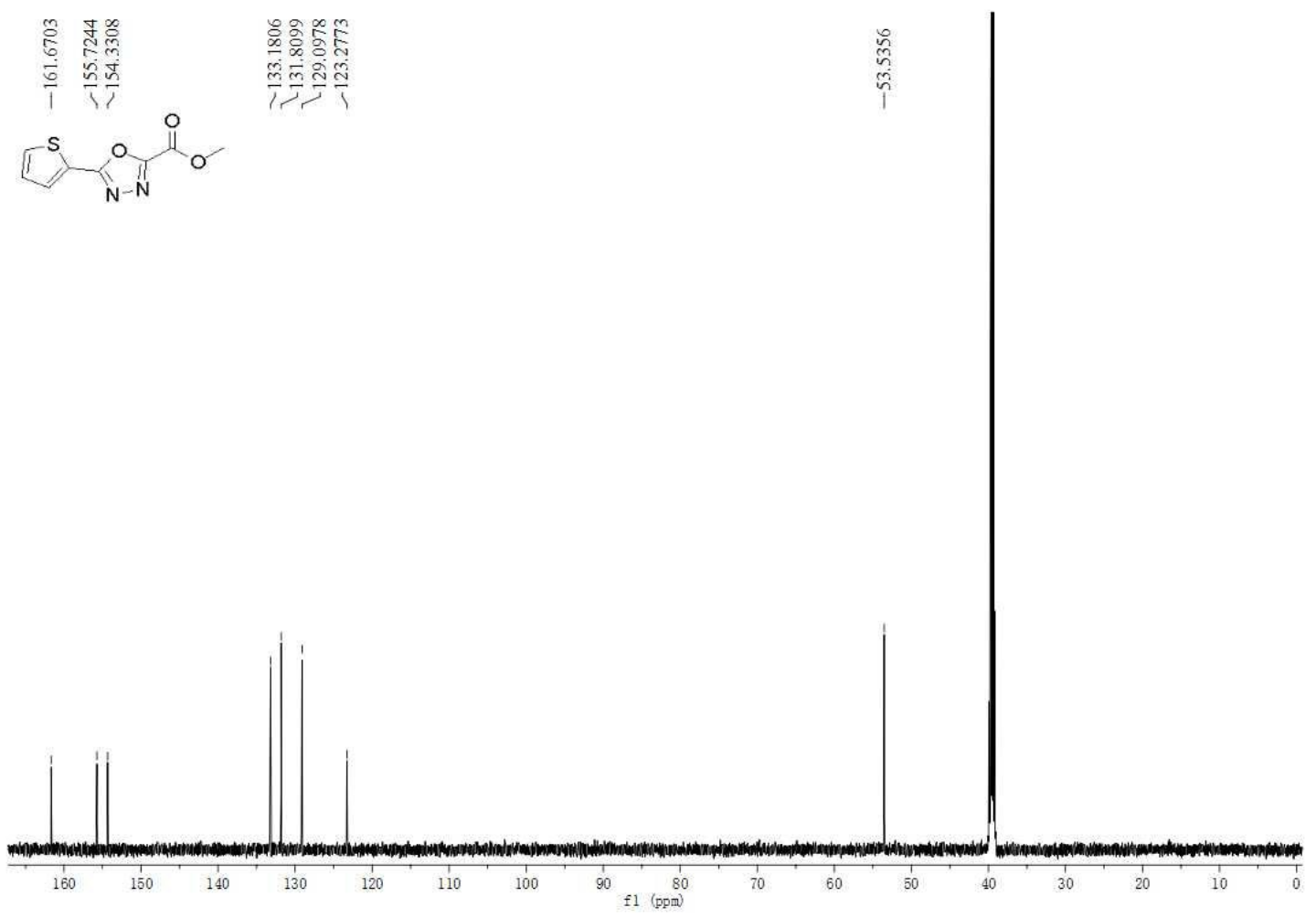

Figure S2. The ${ }^{13} \mathrm{C}$ NMR spectrum $\left(150 \mathrm{MHz}, \mathrm{DMSO}-d_{6}\right)$ of intermediate 3a 

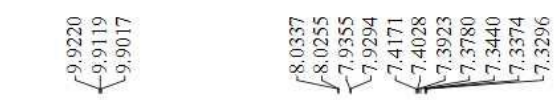
(1) $\int^{n-N}$

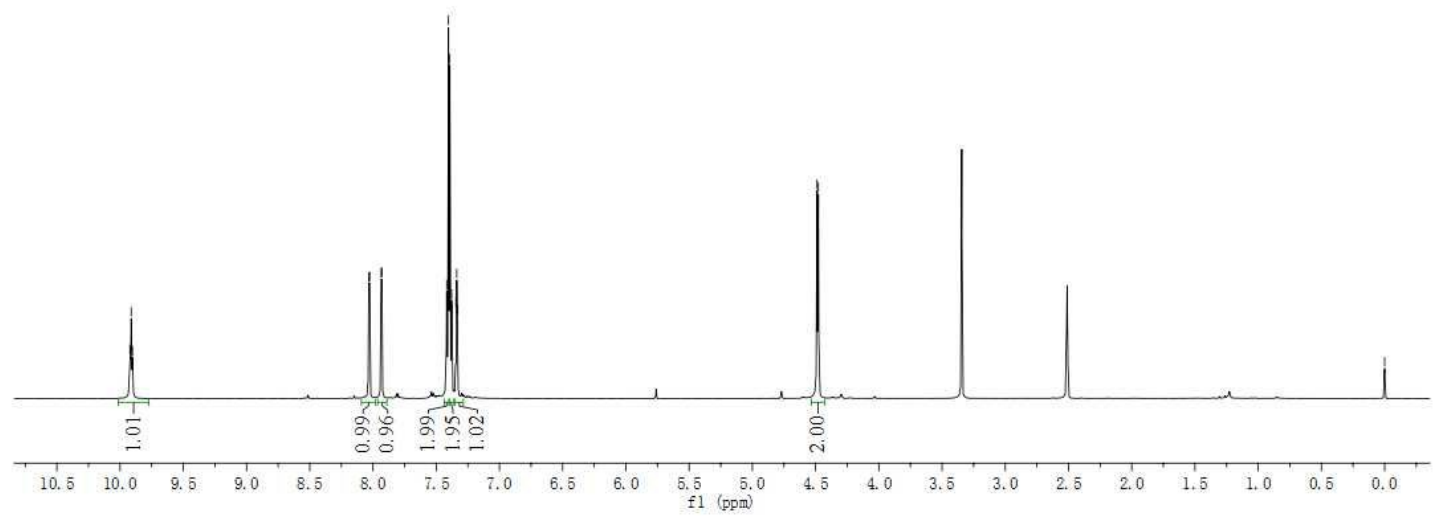

Figure S3. The ${ }^{1} \mathrm{H}$ NMR spectrum (600 MHz, DMSO- $d_{6}$ ) of compound 4a 

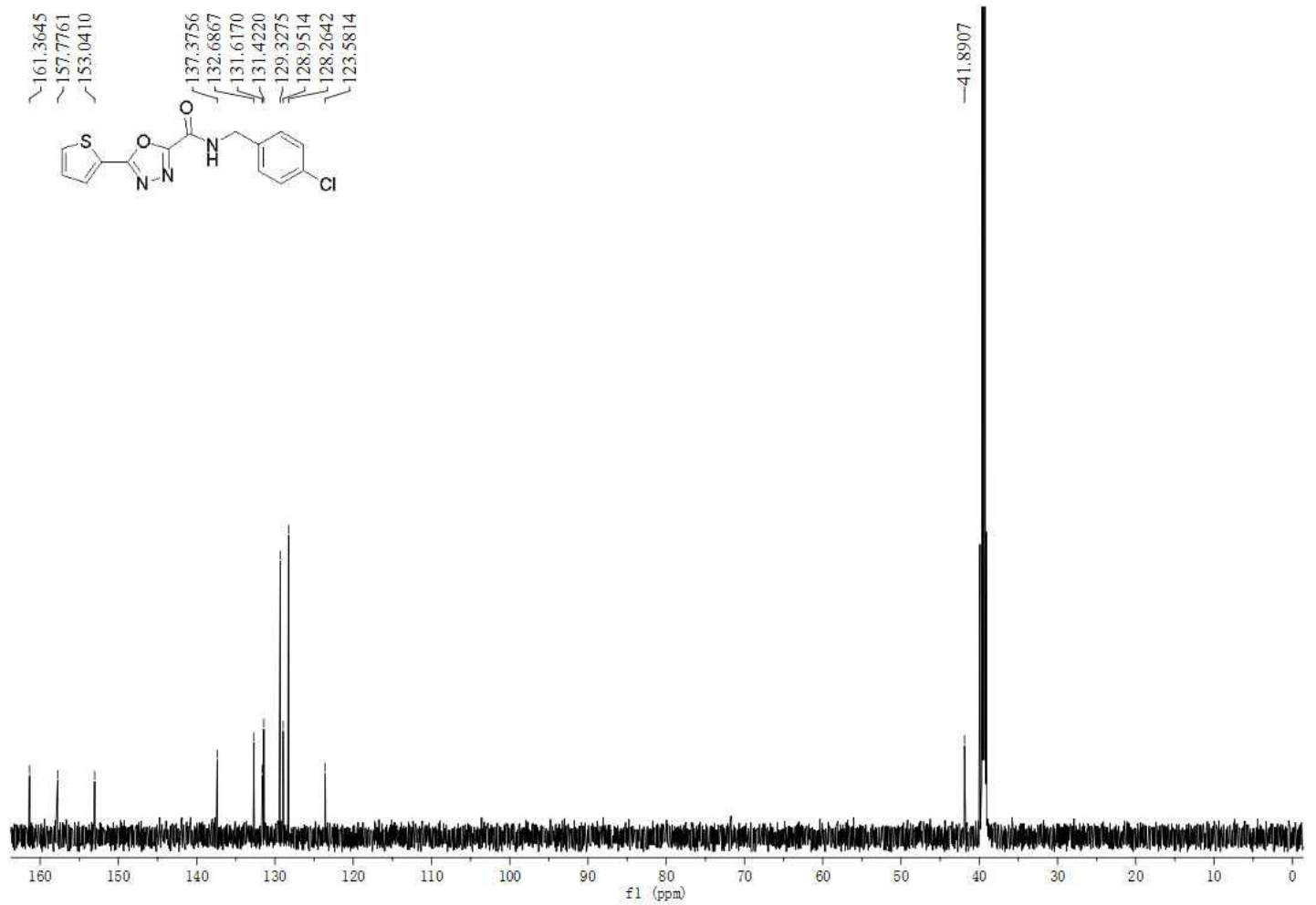

Figure S4. The ${ }^{13} \mathrm{C}$ NMR spectrum (150 MHz, DMSO- $\left.d_{6}\right)$ of compound $4 a$

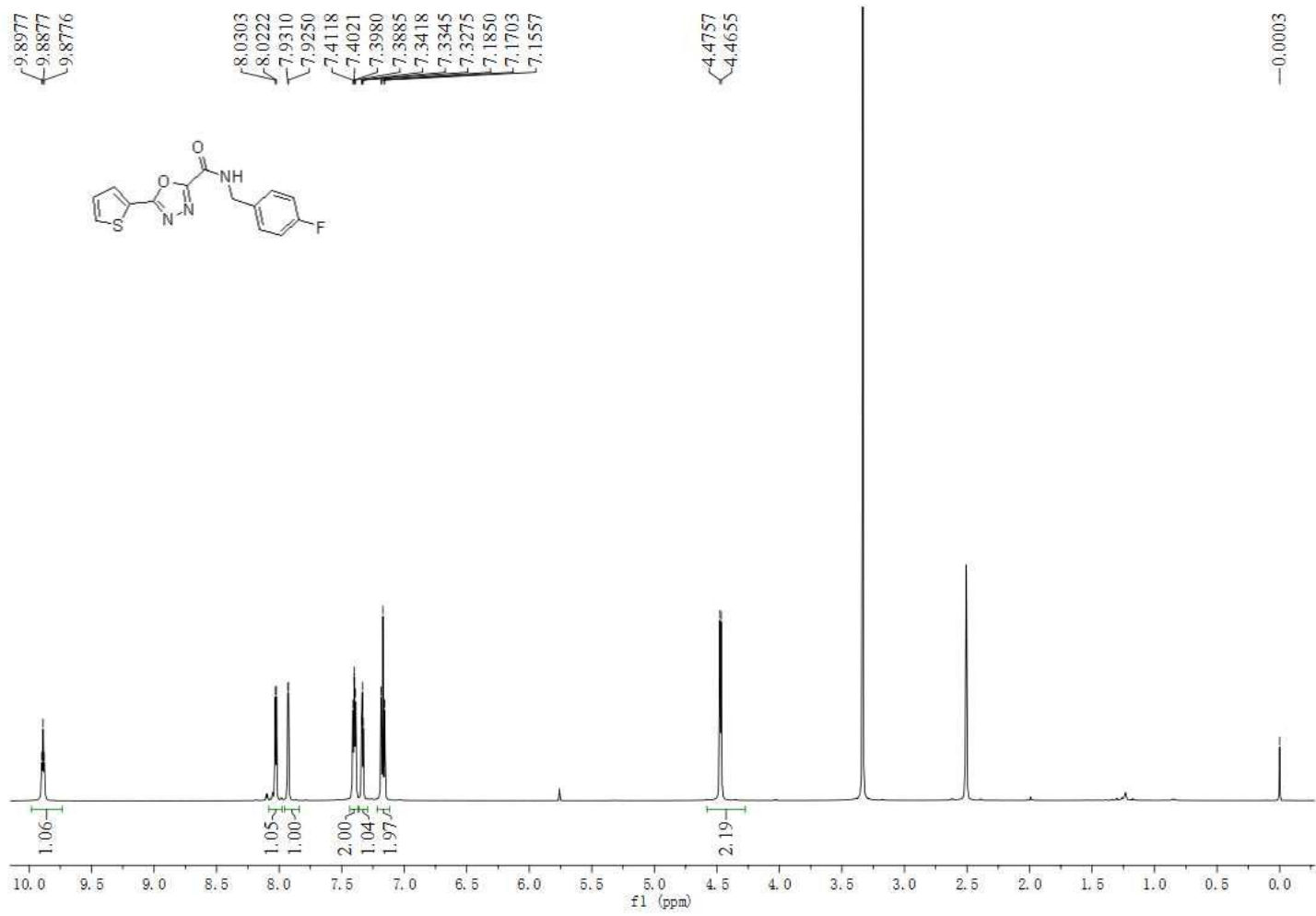

Figure S5. The ${ }^{1} \mathrm{H}$ NMR spectrum $\left(600 \mathrm{MHz}, \mathrm{DMSO}-d_{6}\right)$ of compound $4 \mathbf{b}$ 


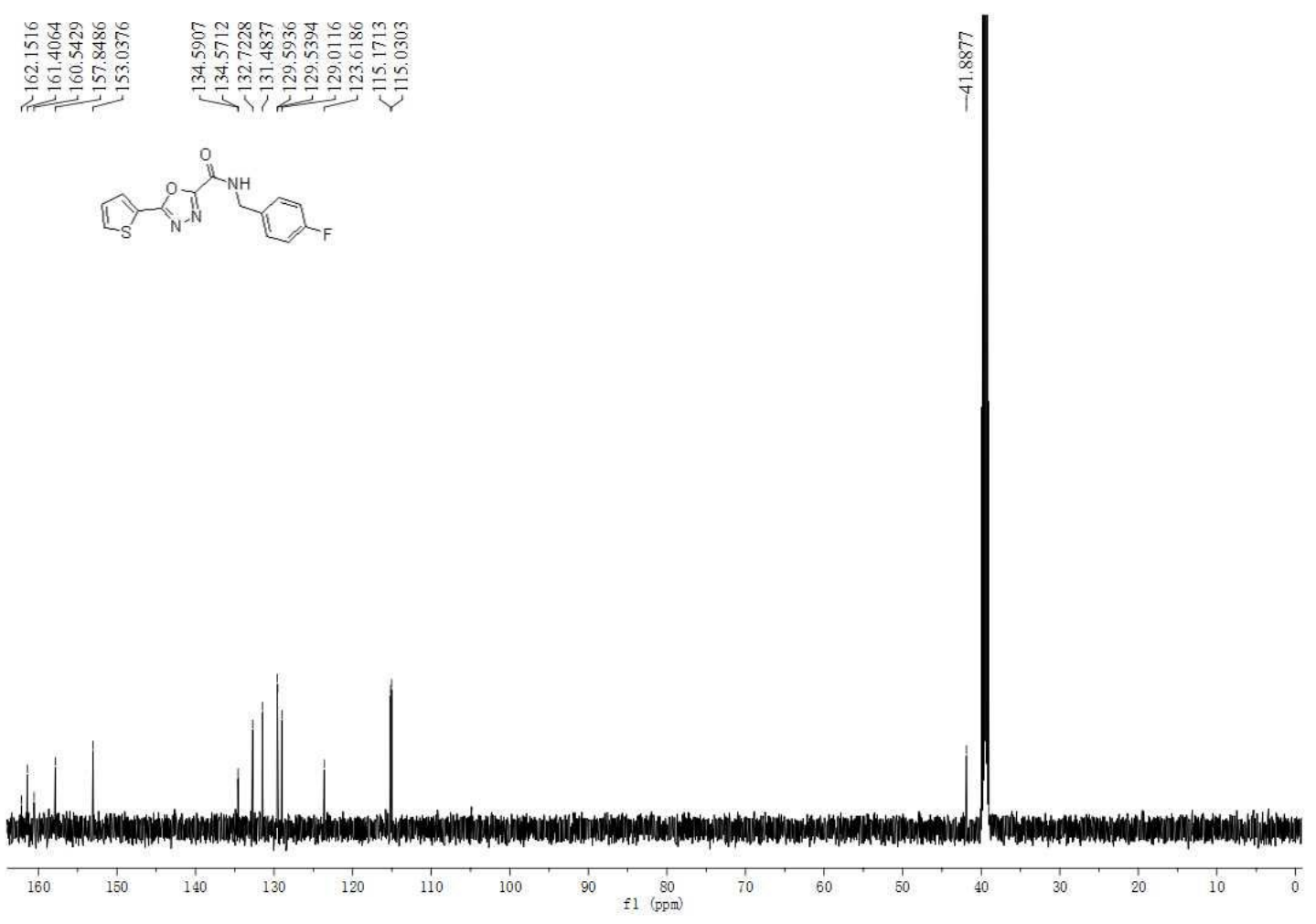

Figure S6. The ${ }^{13} \mathrm{C}$ NMR spectrum (150 MHz, DMSO- $d_{6}$ ) of compound $\mathbf{4 b}$

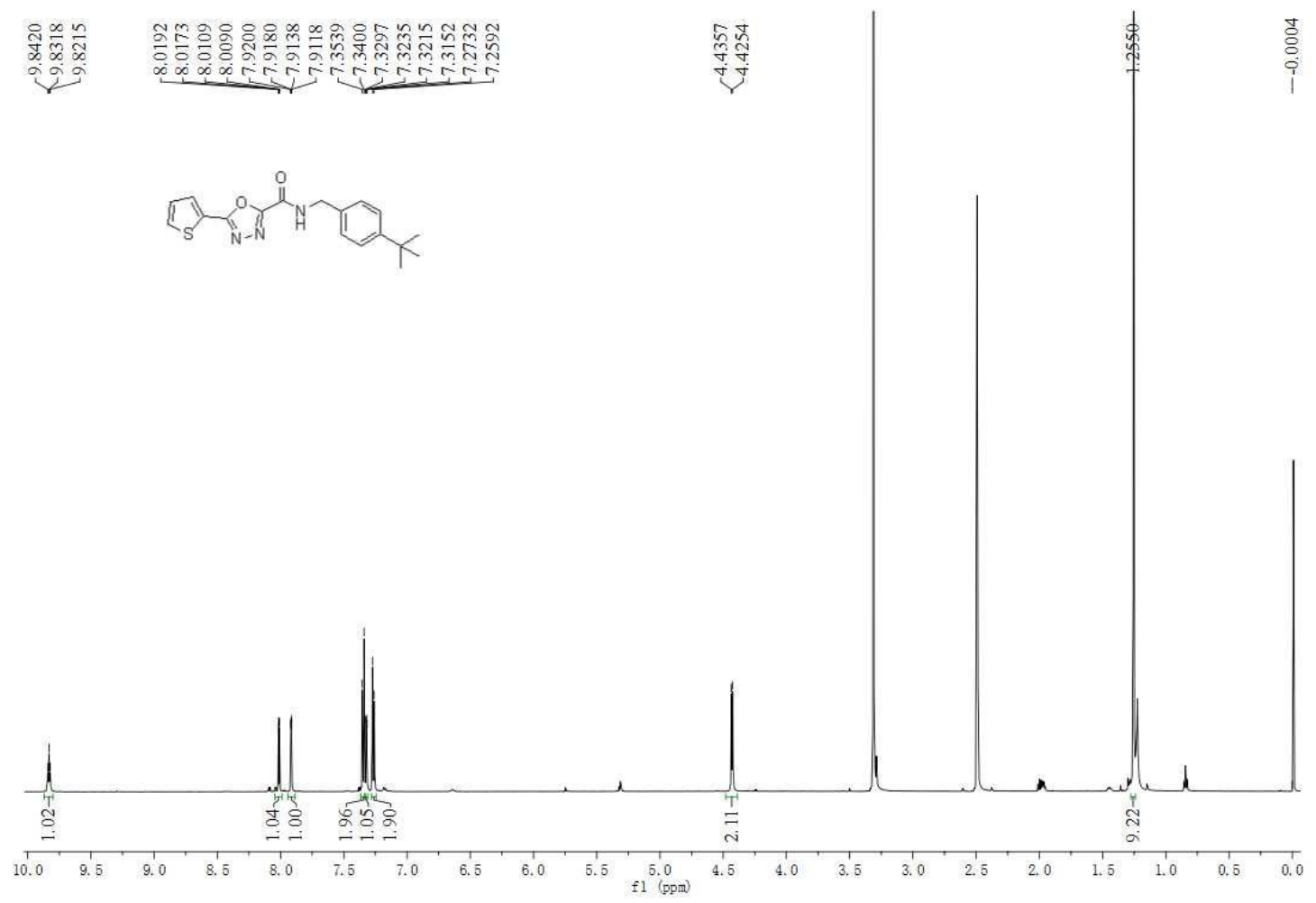

Figure S7. The ${ }^{1} \mathrm{H}$ NMR spectrum $\left(600 \mathrm{MHz}\right.$, DMSO- $\left.d_{6}\right)$ of compound $\mathbf{4 c}$ 


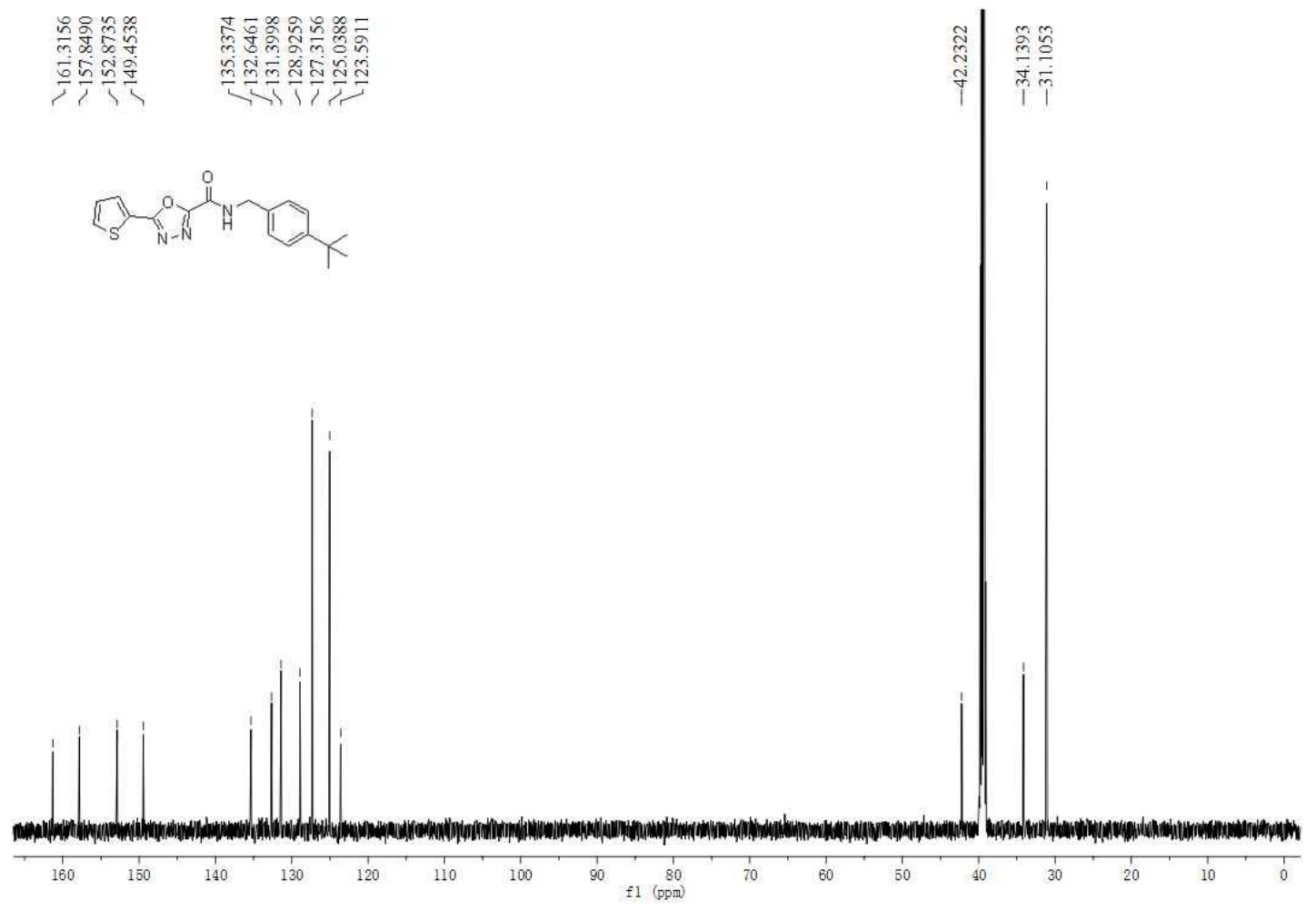

Figure S8. The ${ }^{13} \mathrm{C}$ NMR spectrum (150 MHz, DMSO- $d_{6}$ ) of compound $4 \mathbf{c}$ 


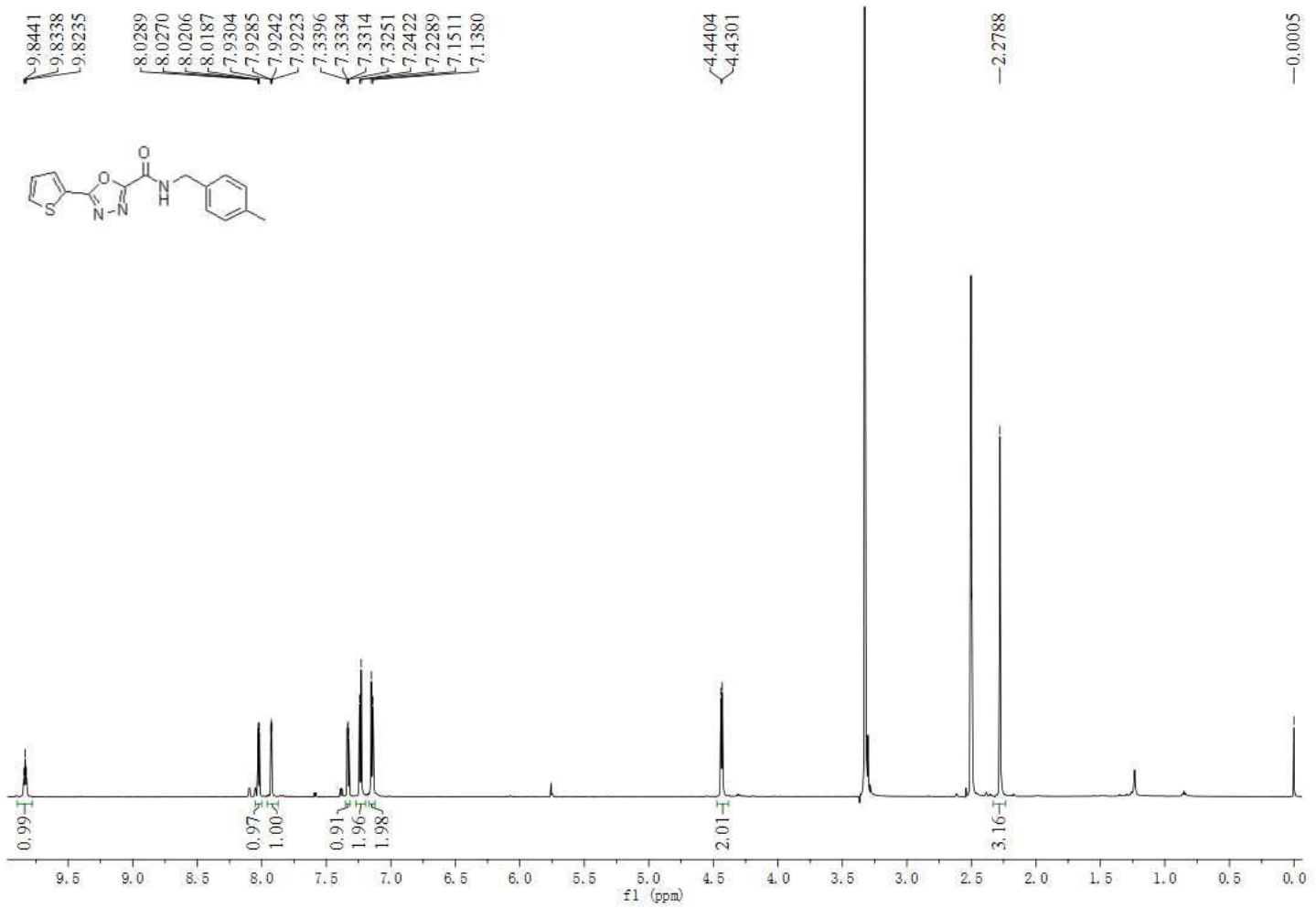

Figure S9. The ${ }^{1} \mathrm{H}$ NMR spectrum $\left(600 \mathrm{MHz}, \mathrm{DMSO}-d_{6}\right)$ of compound $\mathbf{4 d}$
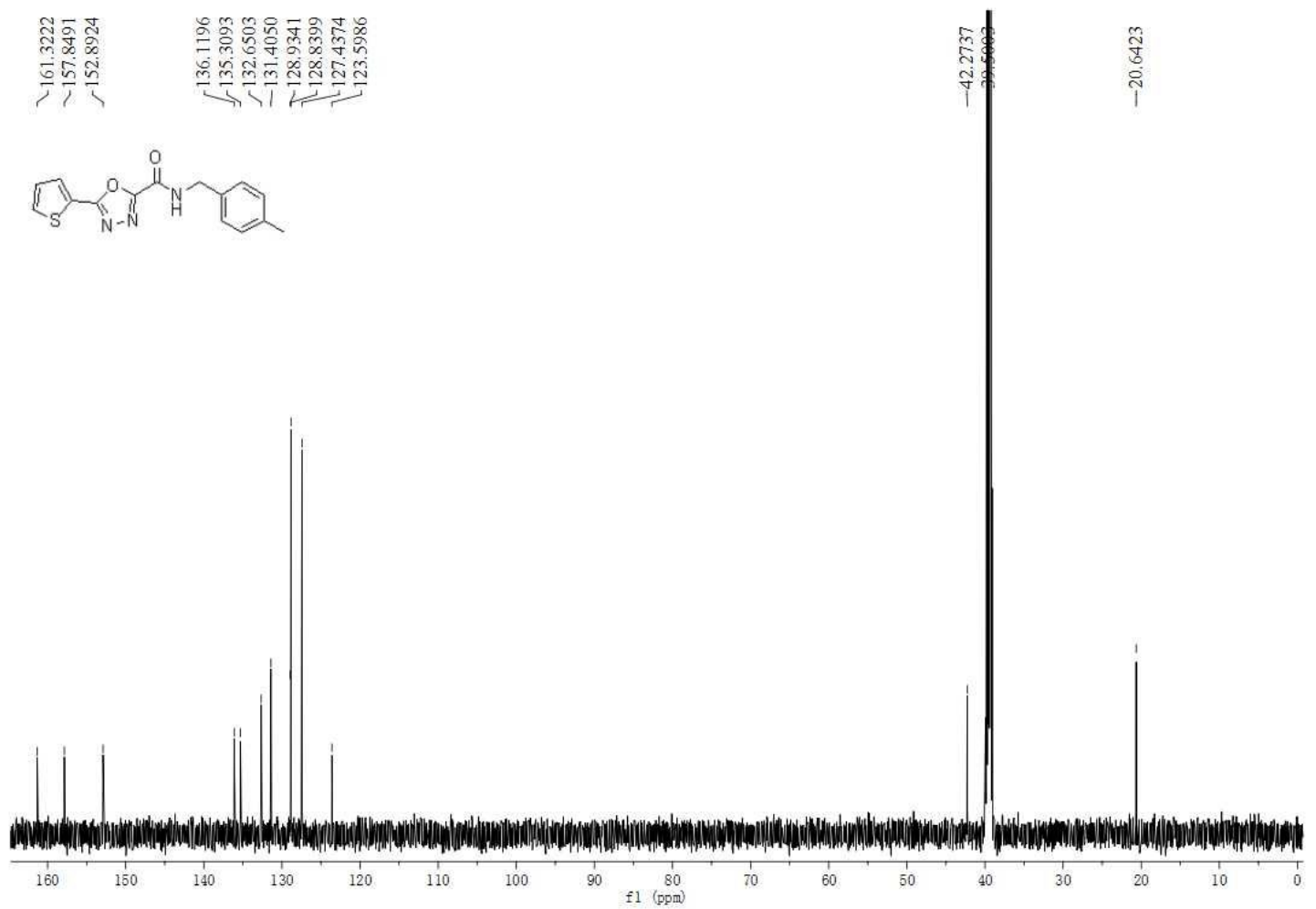

Figure S10. The ${ }^{13} \mathrm{C}$ NMR spectrum $\left(150 \mathrm{MHz}\right.$, DMSO- $\left.d_{6}\right)$ of compound $4 \mathbf{d}$ 


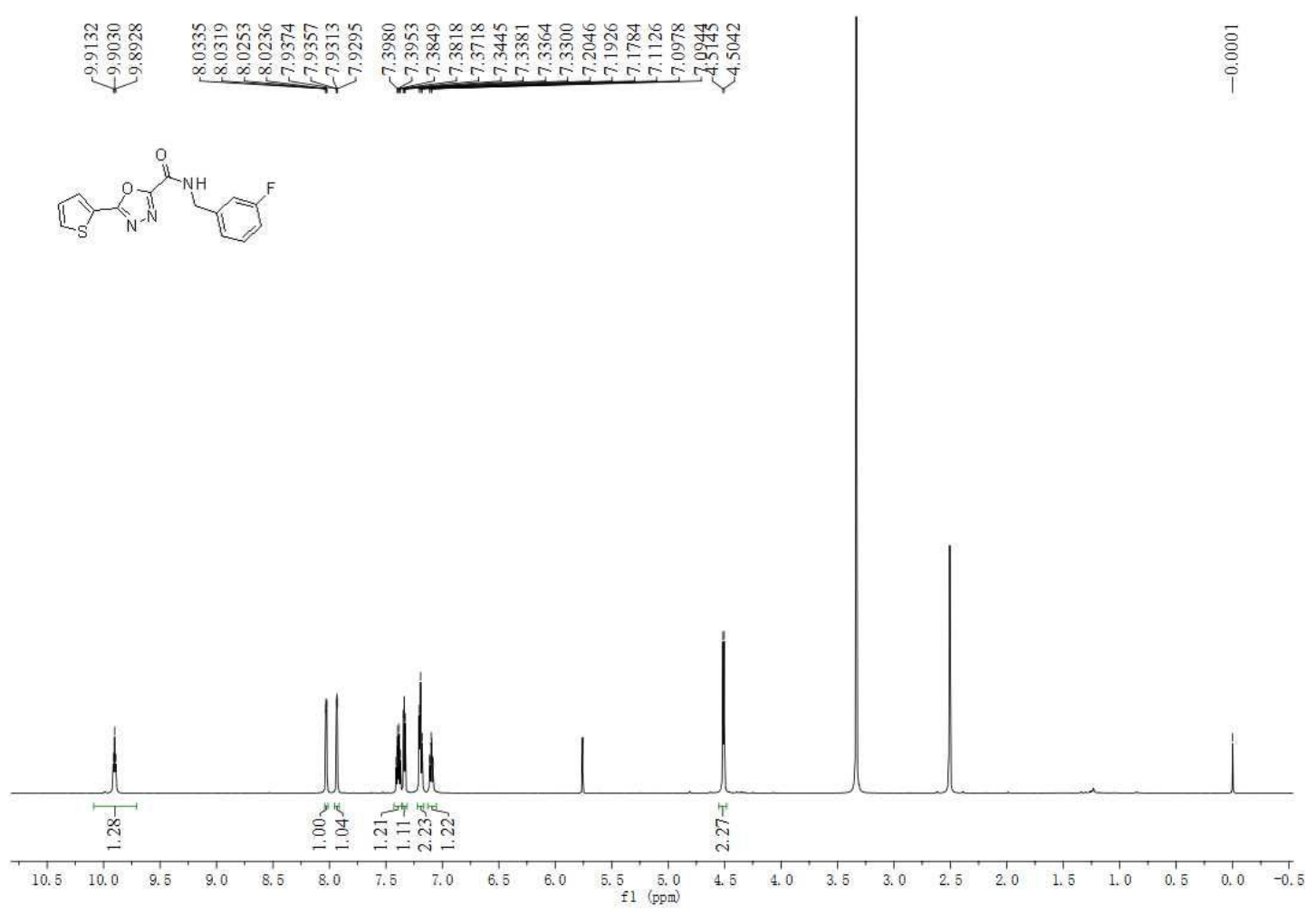

Figure S11. The ${ }^{1} \mathrm{H}$ NMR spectrum $\left(600 \mathrm{MHz}, \mathrm{DMSO}-d_{6}\right)$ of compound $4 \mathbf{e}$ 

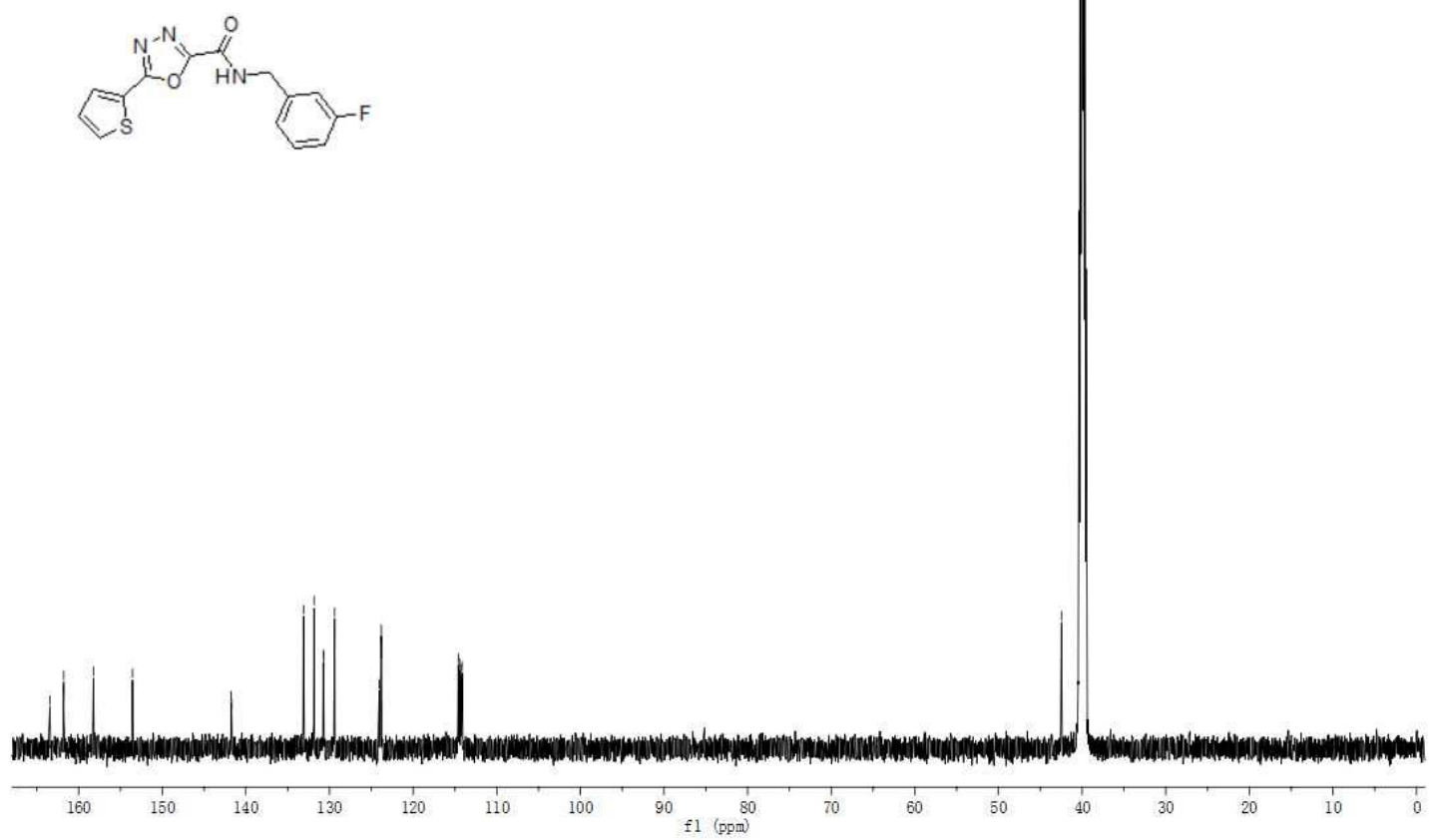

Figure S12. The ${ }^{13} \mathrm{C}$ NMR spectrum $\left(150 \mathrm{MHz}\right.$, DMSO- $\left.d_{6}\right)$ of compound $4 \mathbf{e}$

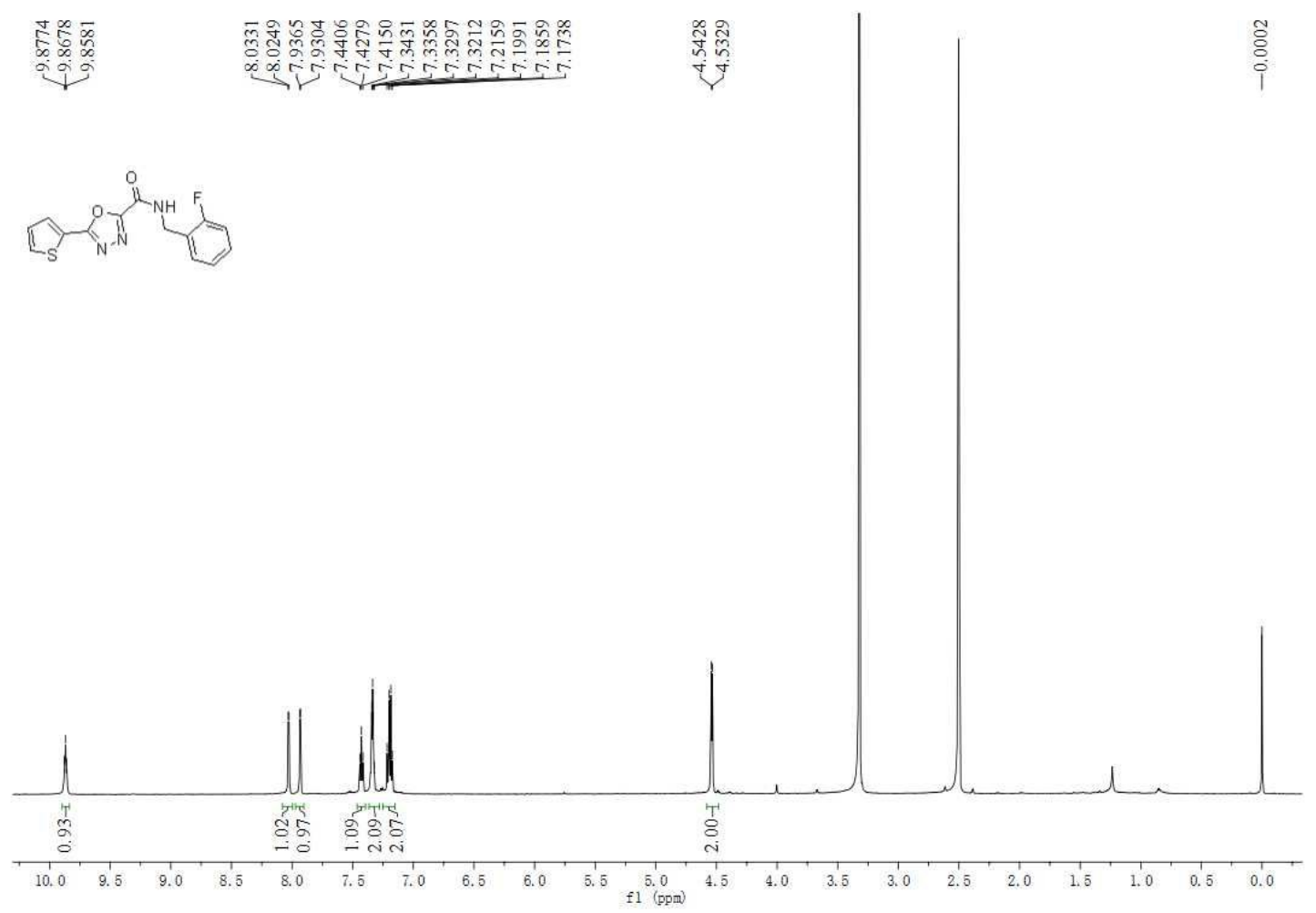

Figure S13. The ${ }^{1} \mathrm{H}$ NMR spectrum $\left(600 \mathrm{MHz}, \mathrm{DMSO}-d_{6}\right)$ of compound $\mathbf{4 f}$ 


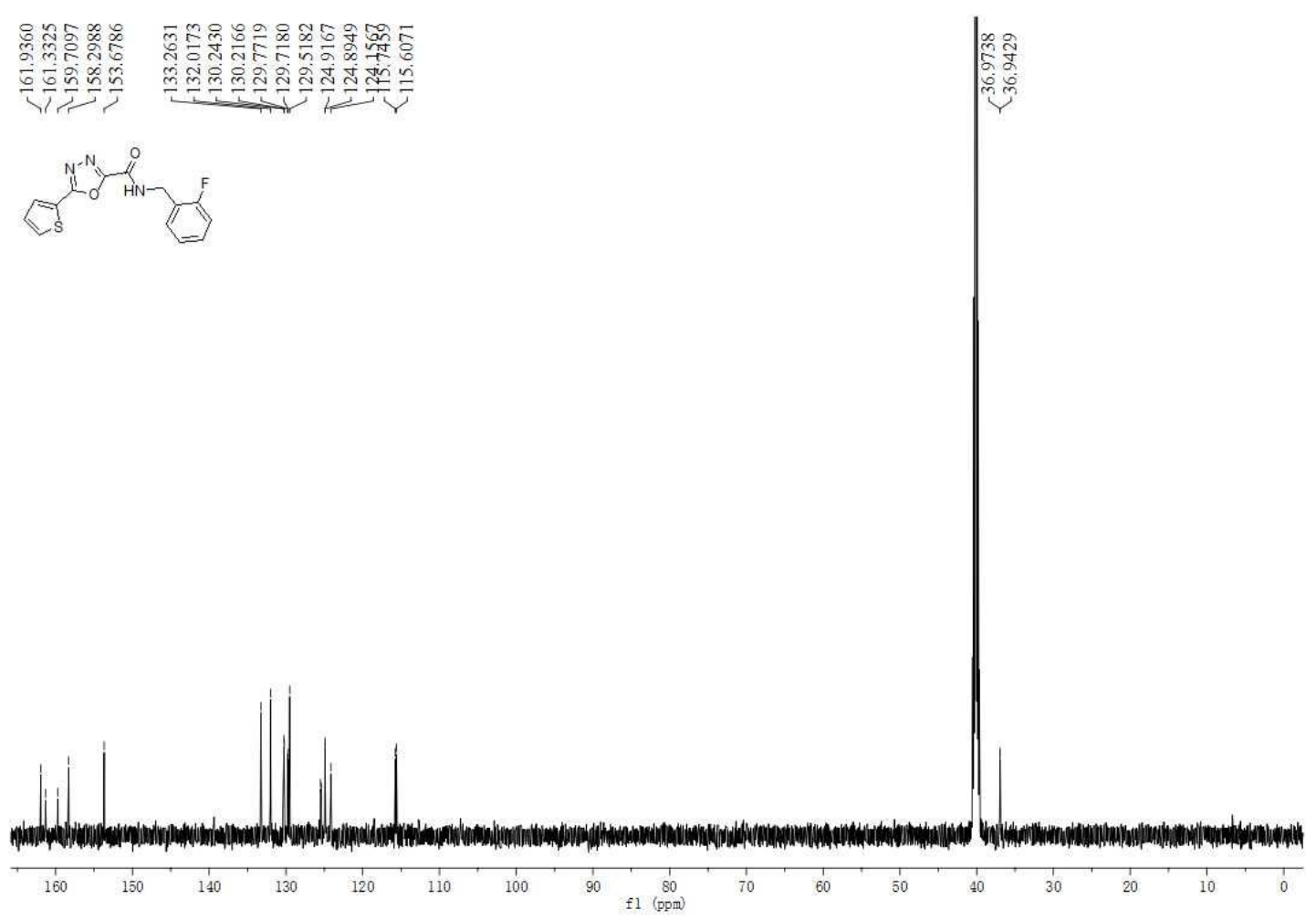

Figure S14. The ${ }^{13} \mathrm{C}$ NMR spectrum $\left(150 \mathrm{MHz}, \mathrm{DMSO}-d_{6}\right)$ of compound $\mathbf{4 f}$ 


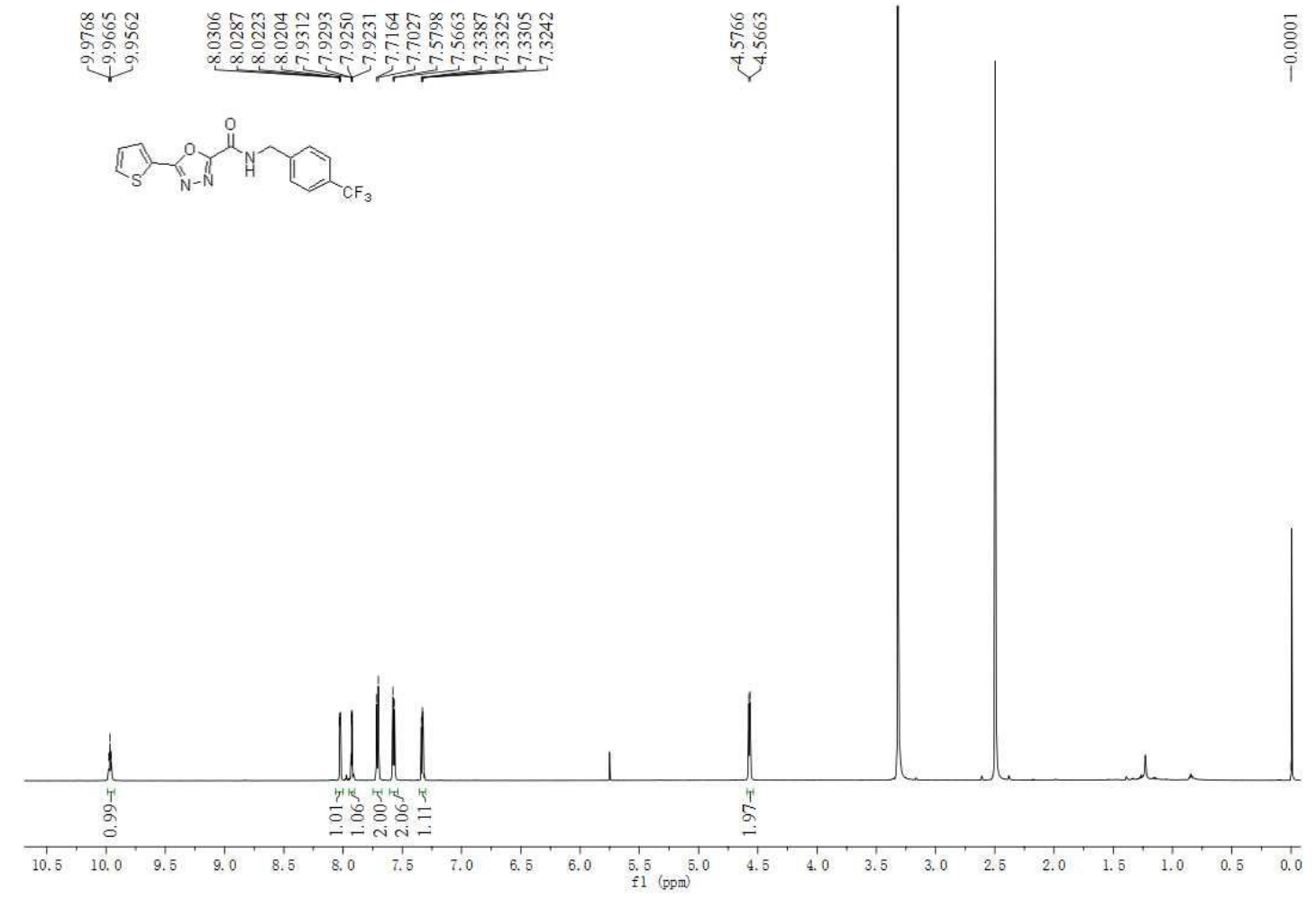

Figure S15. The ${ }^{1} \mathrm{H}$ NMR spectrum (600 MHz, DMSO- $d_{6}$ ) of compound $\mathbf{4 g}$
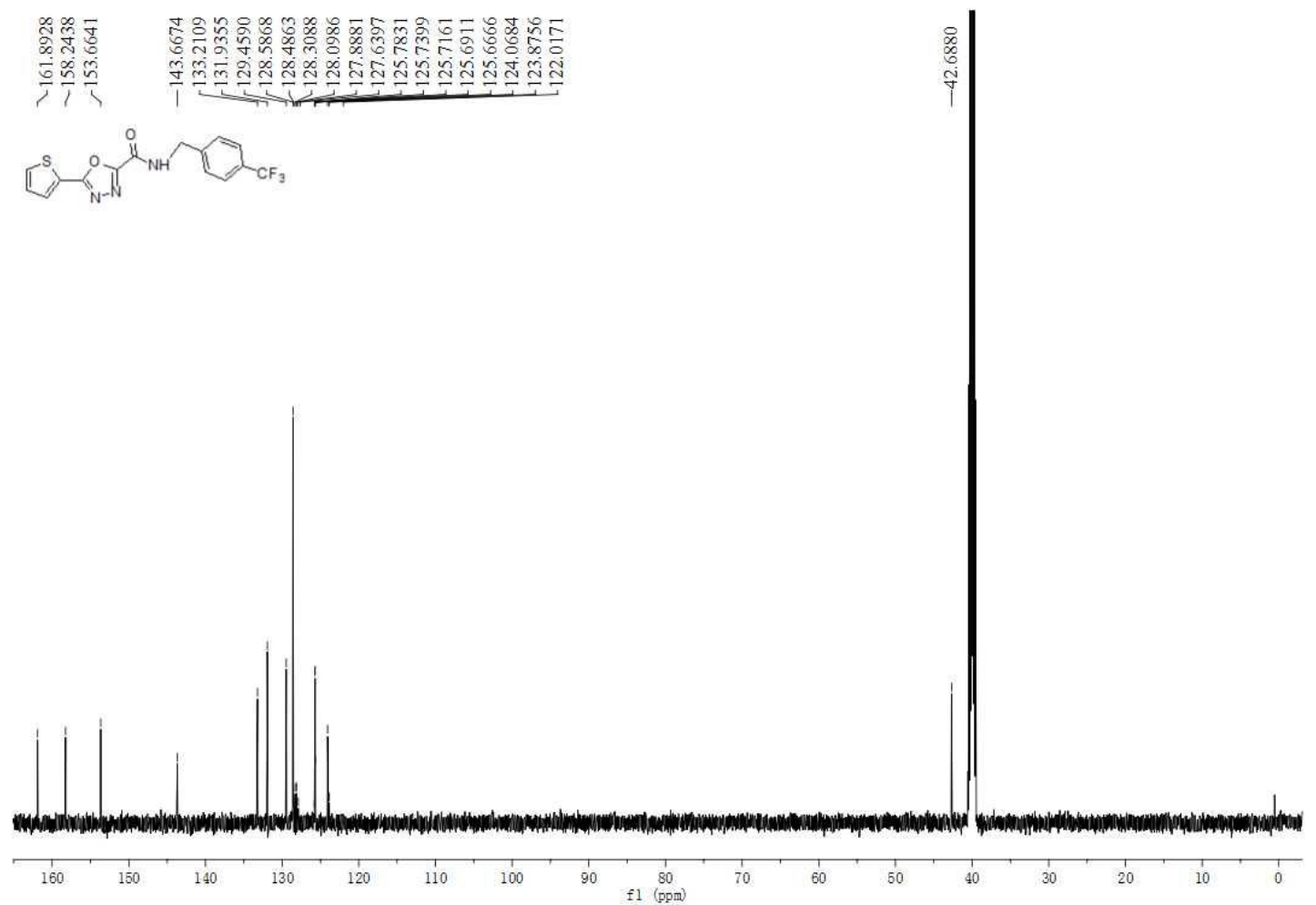

Figure S16. The ${ }^{13} \mathrm{C}$ NMR spectrum $\left(150 \mathrm{MHz}\right.$, DMSO- $\left.d_{6}\right)$ of compound $\mathbf{4 g}$ 


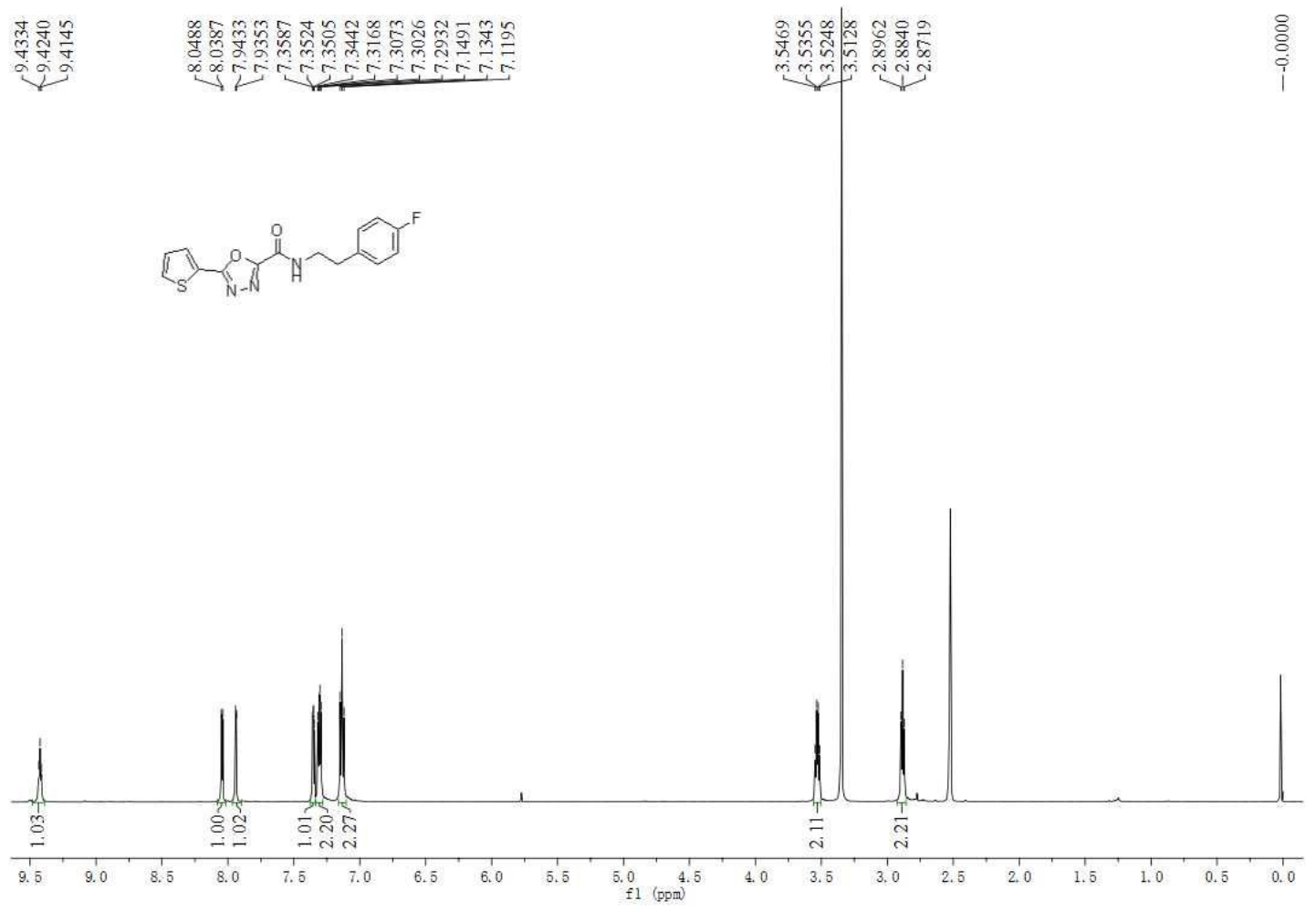

Figure S17. The ${ }^{1} \mathrm{H}$ NMR spectrum (600 MHz, DMSO- $d_{6}$ ) of compound $\mathbf{4 h}$ 

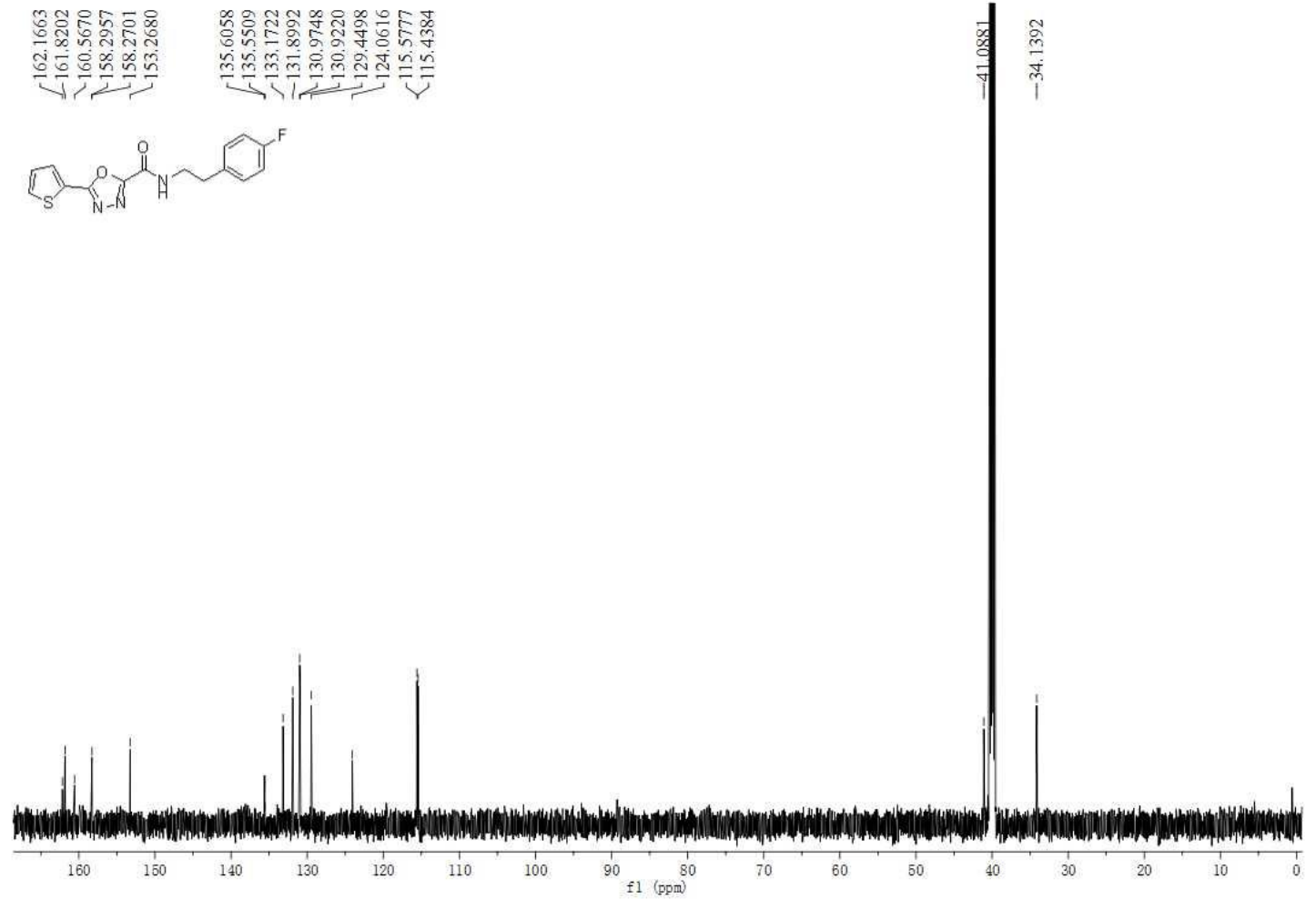

Figure S18. The ${ }^{13} \mathrm{C}$ NMR spectrum $\left(150 \mathrm{MHz}\right.$, DMSO- $\left.d_{6}\right)$ of compound $\mathbf{4 h}$

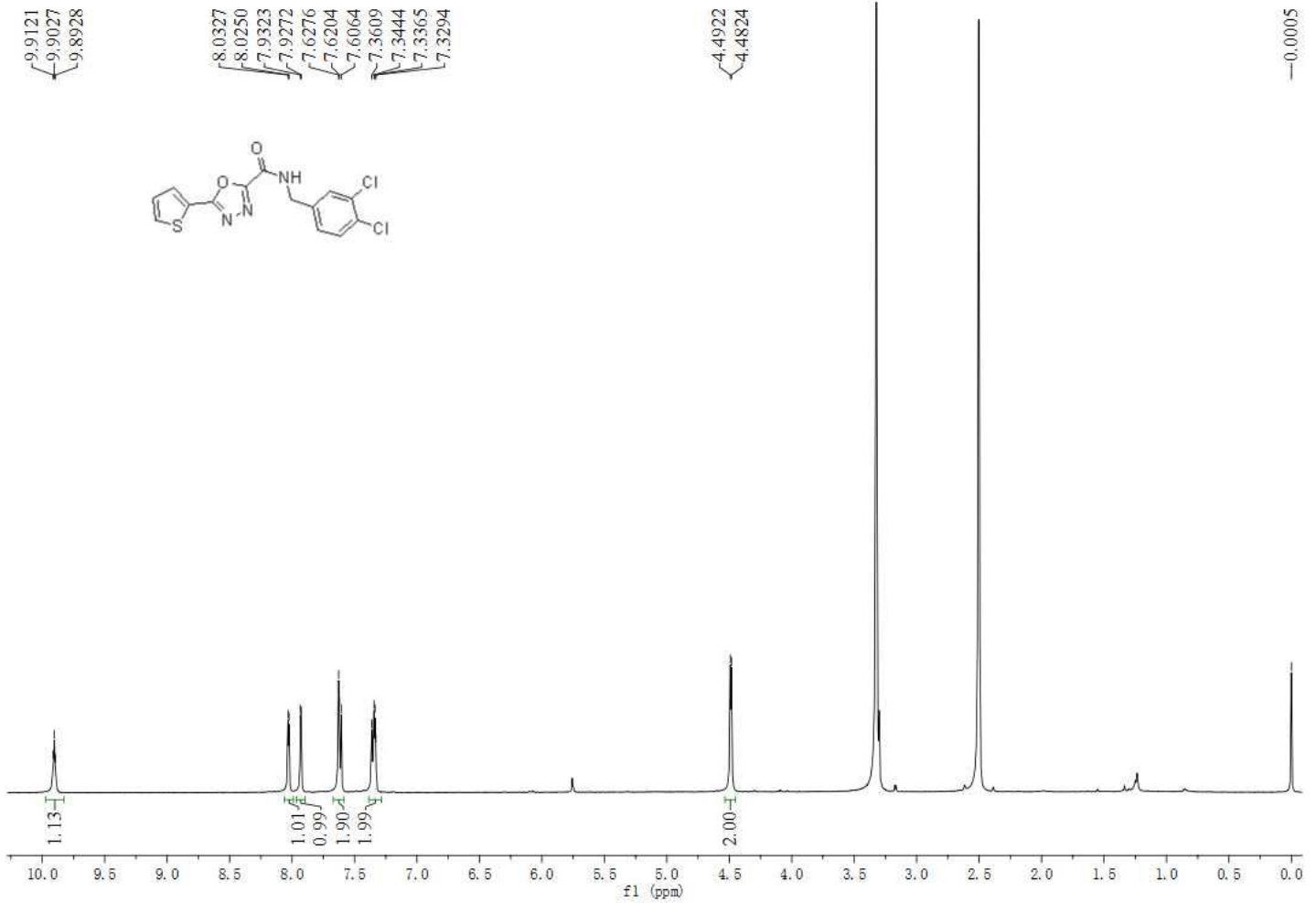

Figure S19. The ${ }^{1} \mathrm{H}$ NMR spectrum (600 MHz, DMSO- $d_{6}$ ) of compound $4 \mathbf{i}$ 


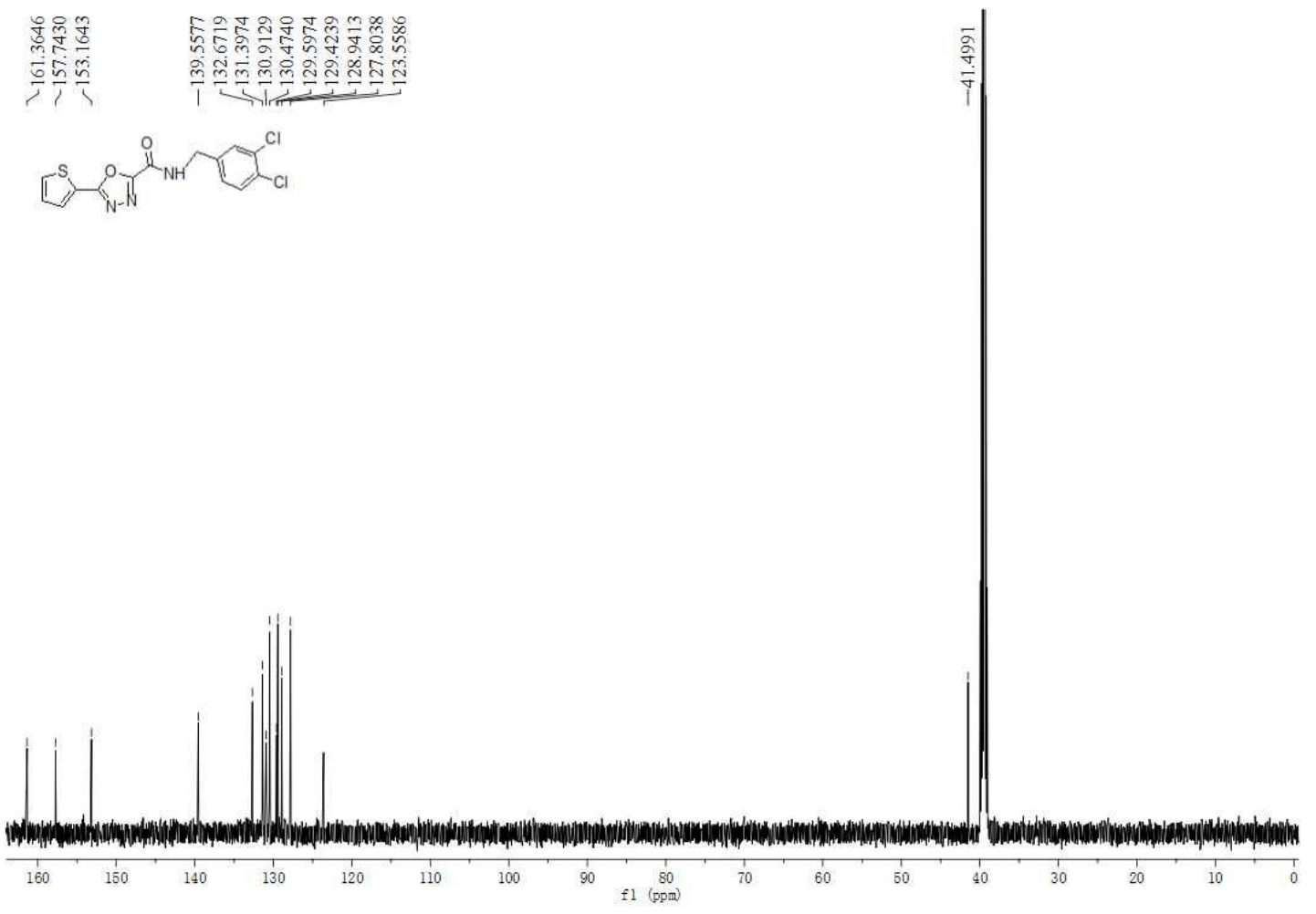

Figure S20. The ${ }^{13} \mathrm{C}$ NMR spectrum (150 MHz, DMSO- $\left.d_{6}\right)$ of compound $4 \mathbf{i}$ 

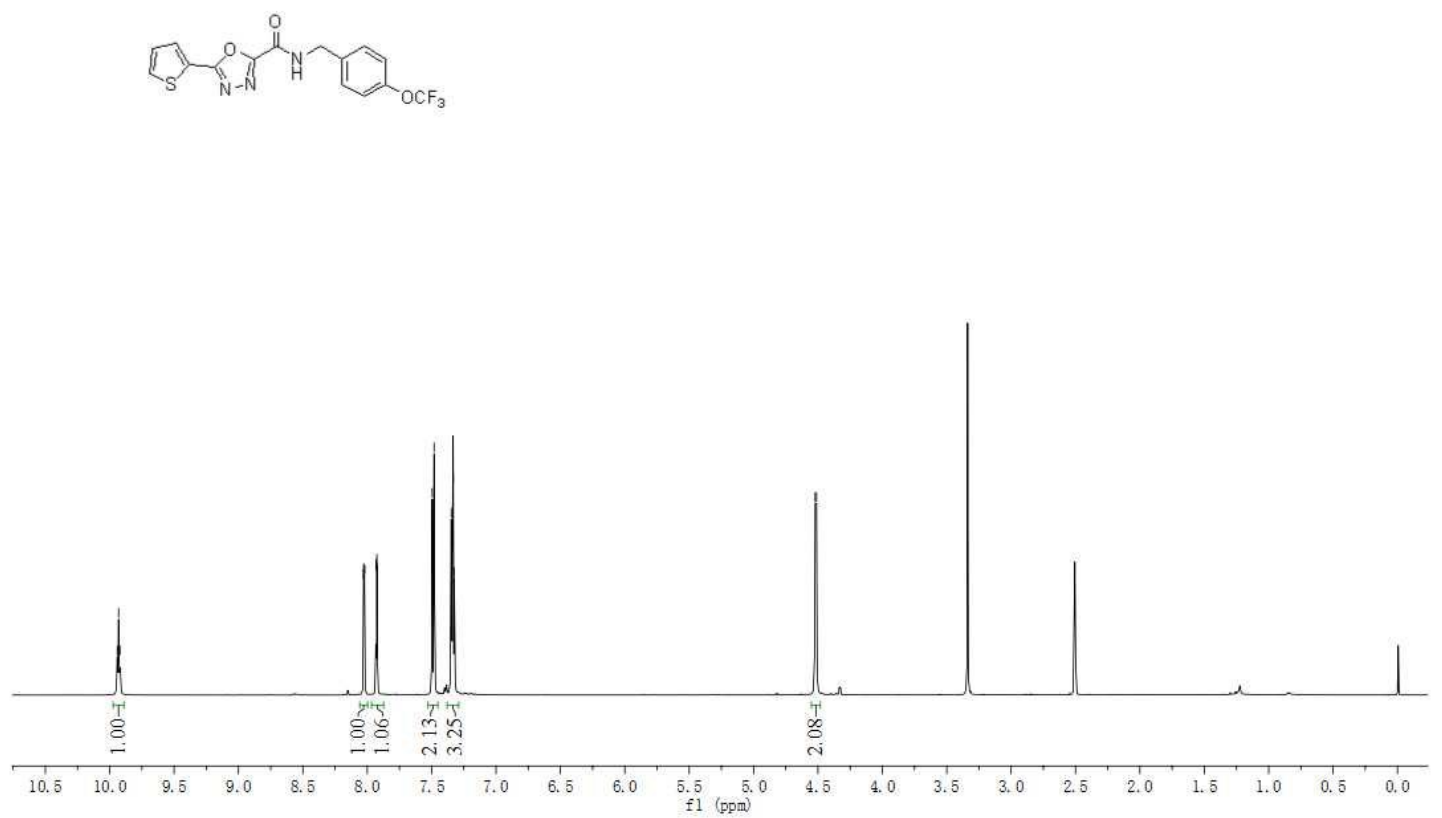

Figure S21. The ${ }^{1} \mathrm{H}$ NMR spectrum (600 MHz, DMSO- $d_{6}$ ) of compound $\mathbf{4 j}$
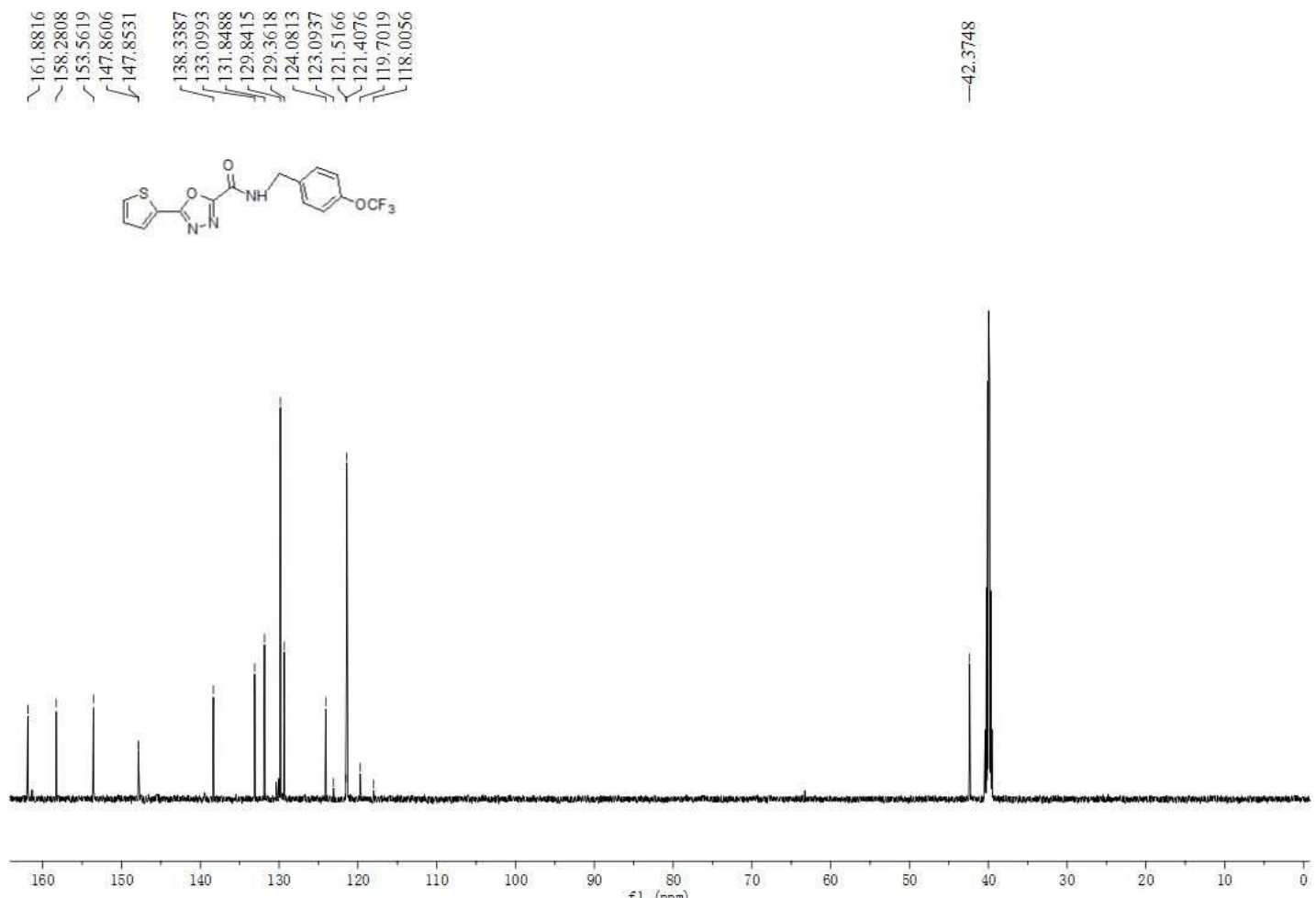

Figure S22. The ${ }^{13} \mathrm{C}$ NMR spectrum (150 MHz, DMSO- $d_{6}$ ) of compound $\mathbf{4 j}$ 


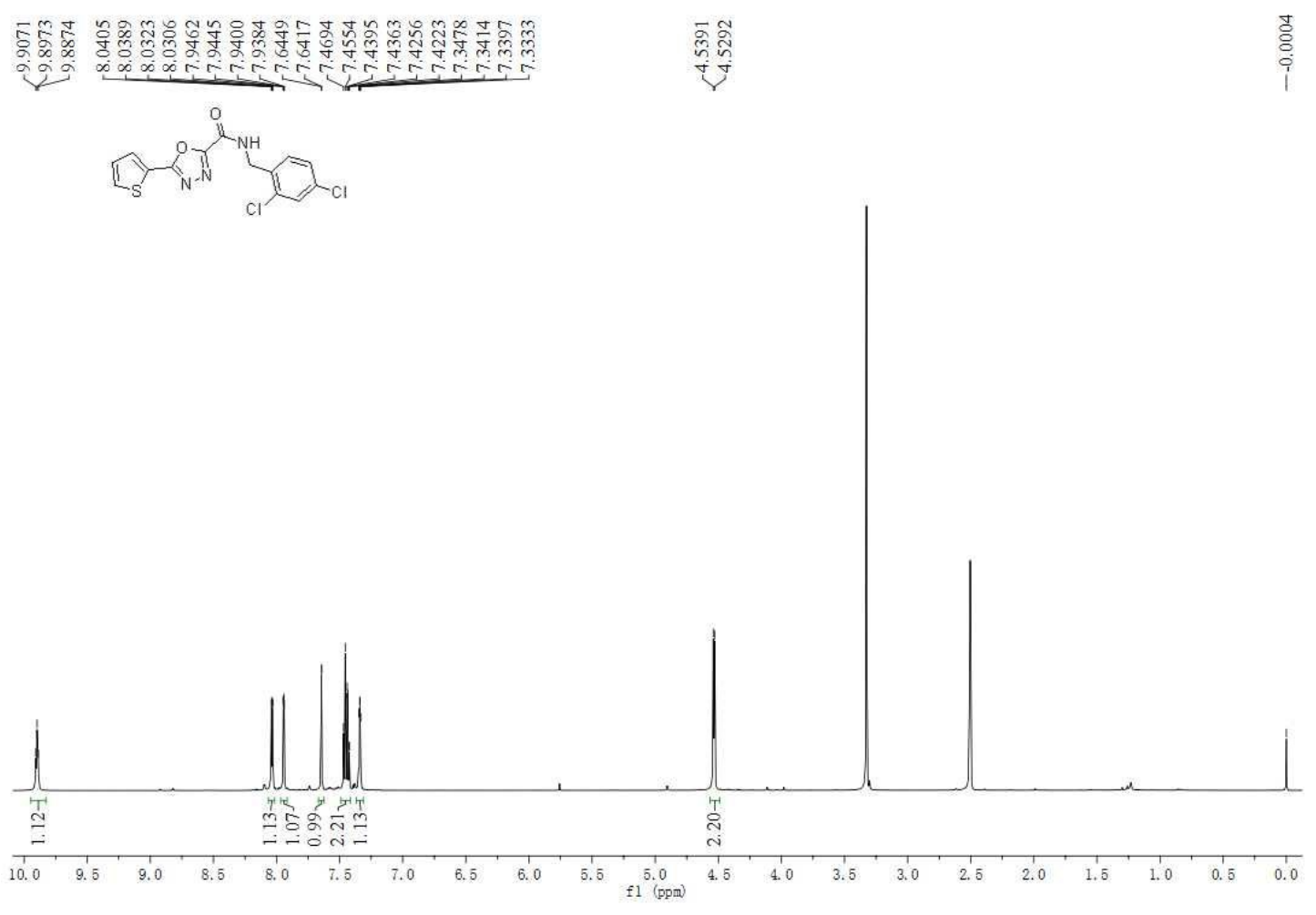

Figure S23. The ${ }^{1} \mathrm{H}$ NMR spectrum (600 MHz, DMSO- $\left.d_{6}\right)$ of compound $4 \mathbf{k}$ 

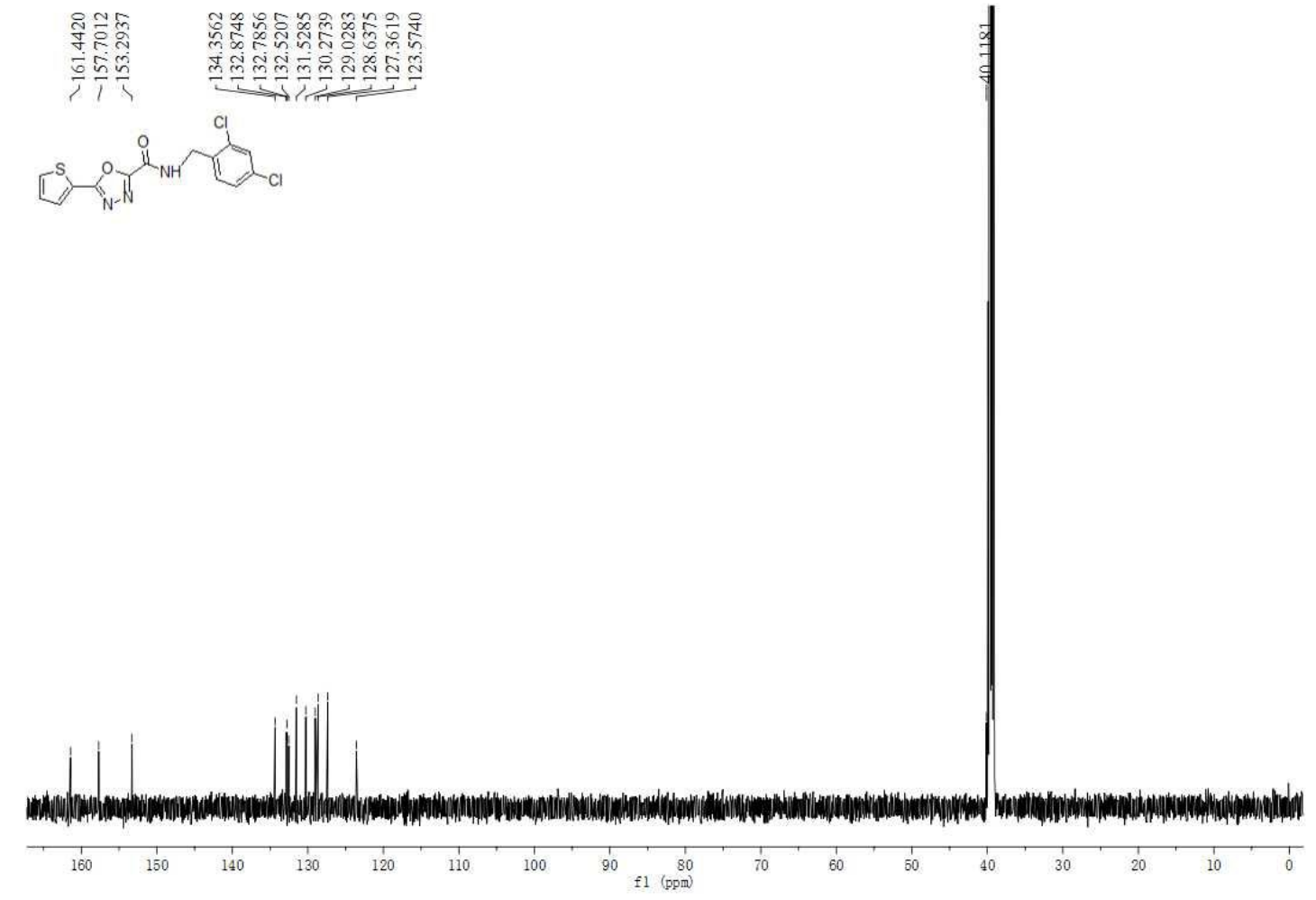

Figure S24. The ${ }^{13} \mathrm{C}$ NMR spectrum $\left(150 \mathrm{MHz}\right.$, DMSO- $\left.d_{6}\right)$ of compound $\mathbf{4 k}$

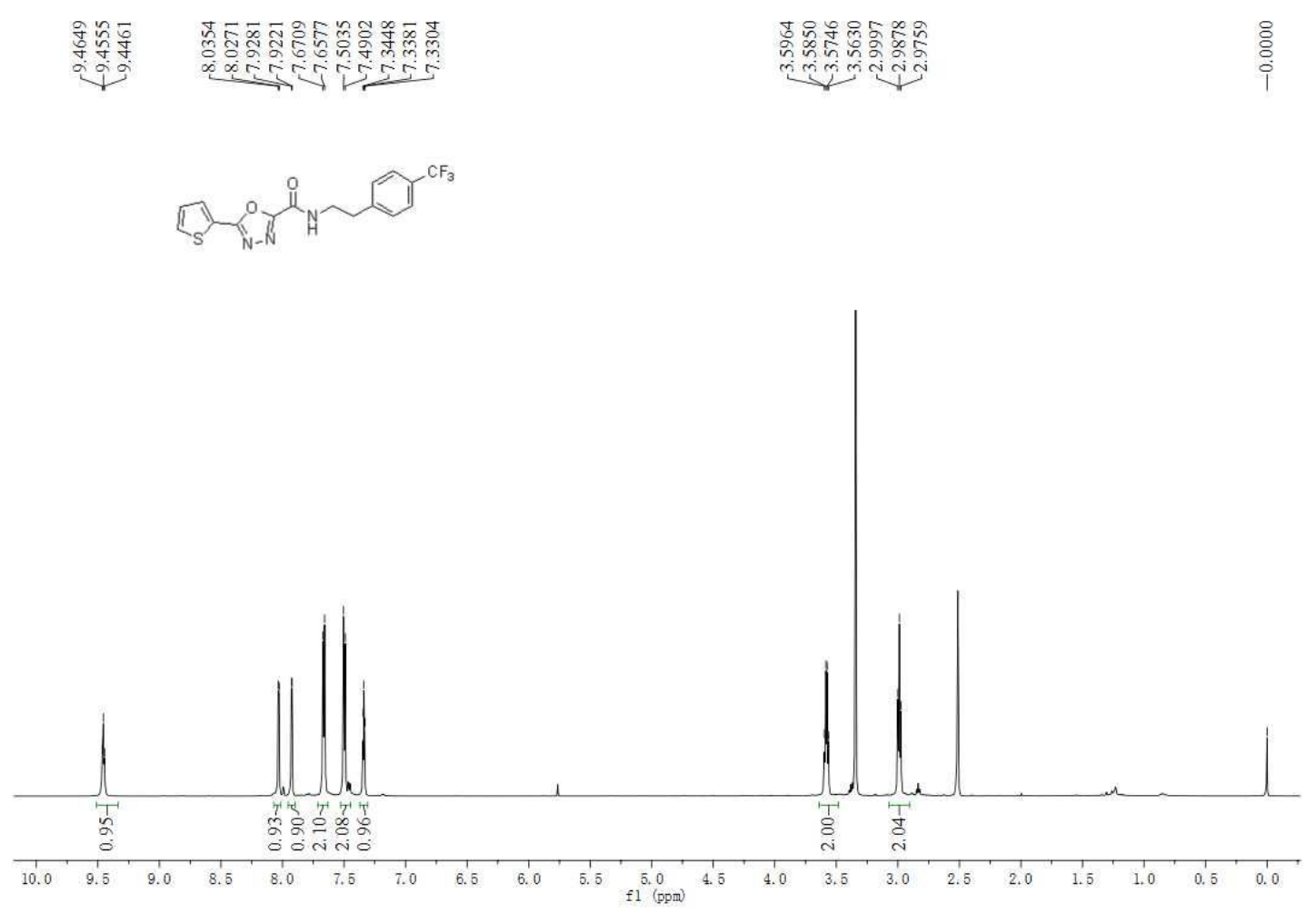

Figure S25. The ${ }^{1} \mathrm{H}$ NMR spectrum (600 MHz, DMSO- $d_{6}$ ) of compound $\mathbf{4 l}$ 


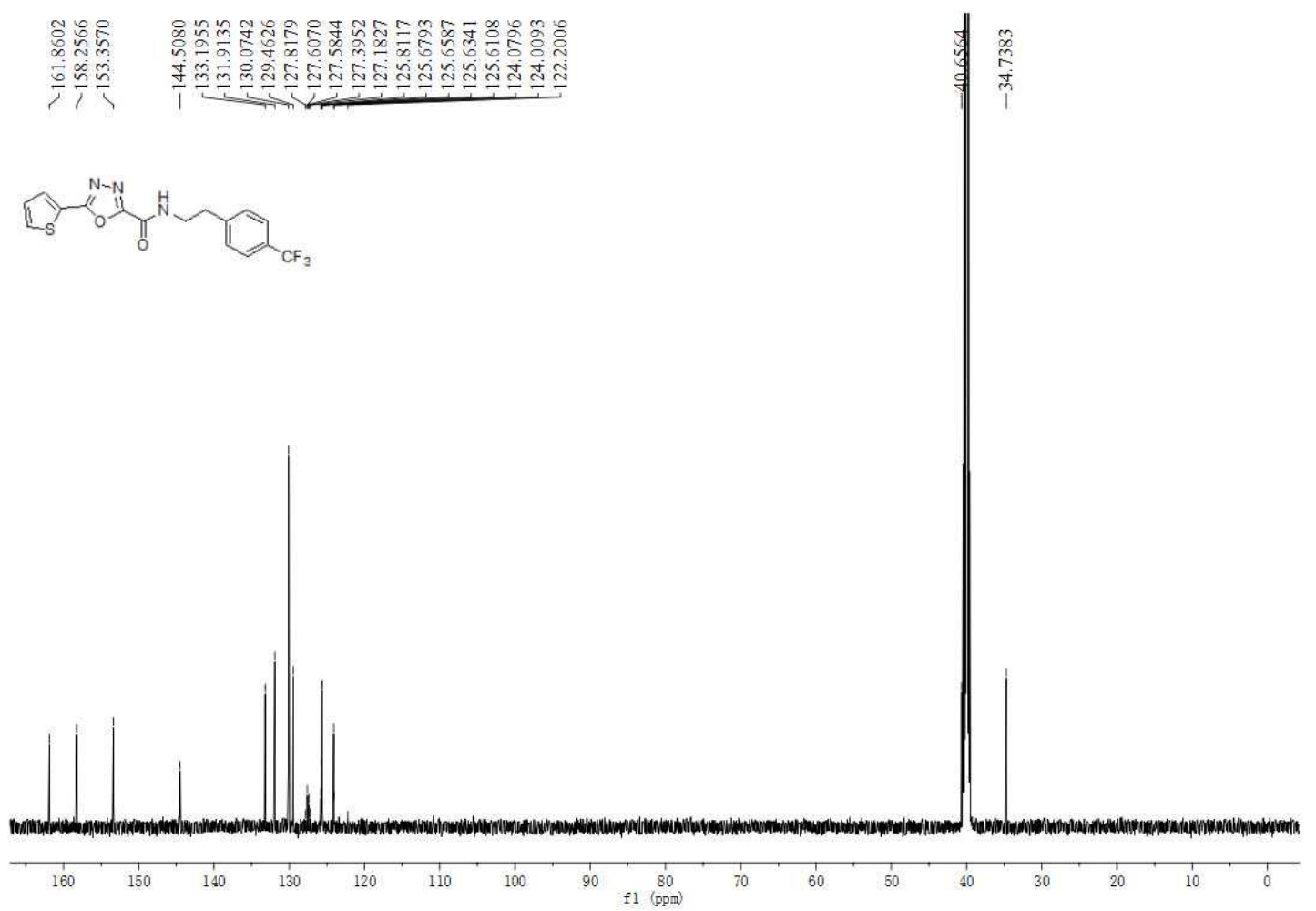

Figure S26. The ${ }^{13} \mathrm{C}$ NMR spectrum (150 MHz, DMSO- $\left.d_{6}\right)$ of compound $4 \mathrm{I}$ 


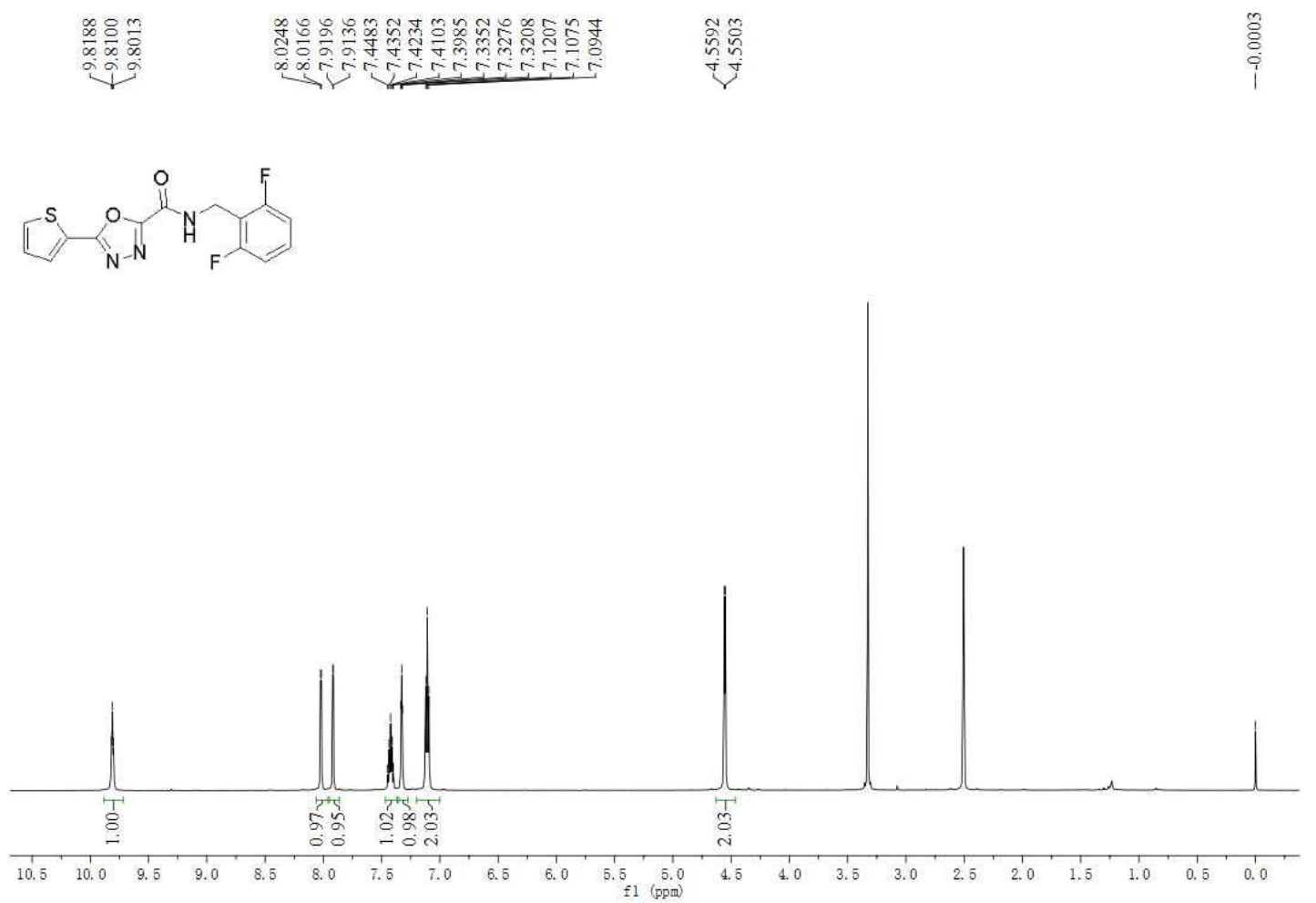

Figure S27. The ${ }^{1} \mathrm{H}$ NMR spectrum (600 MHz, DMSO- $d_{6}$ ) of compound $4 \mathbf{m}$ 


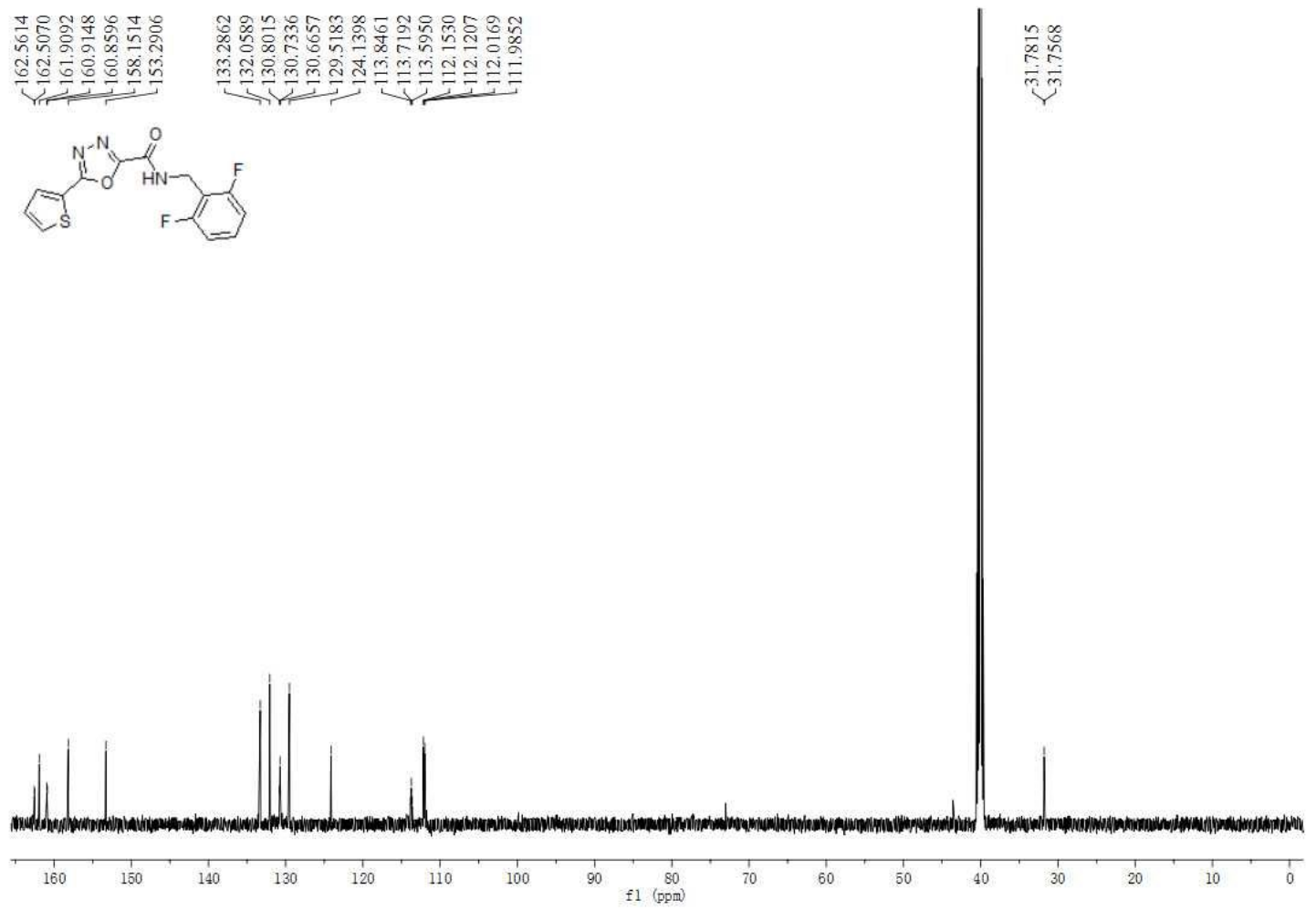

Figure S28. The ${ }^{13} \mathrm{C}$ NMR spectrum (150 MHz, DMSO- $d_{6}$ ) of compound $4 \mathbf{m}$ 


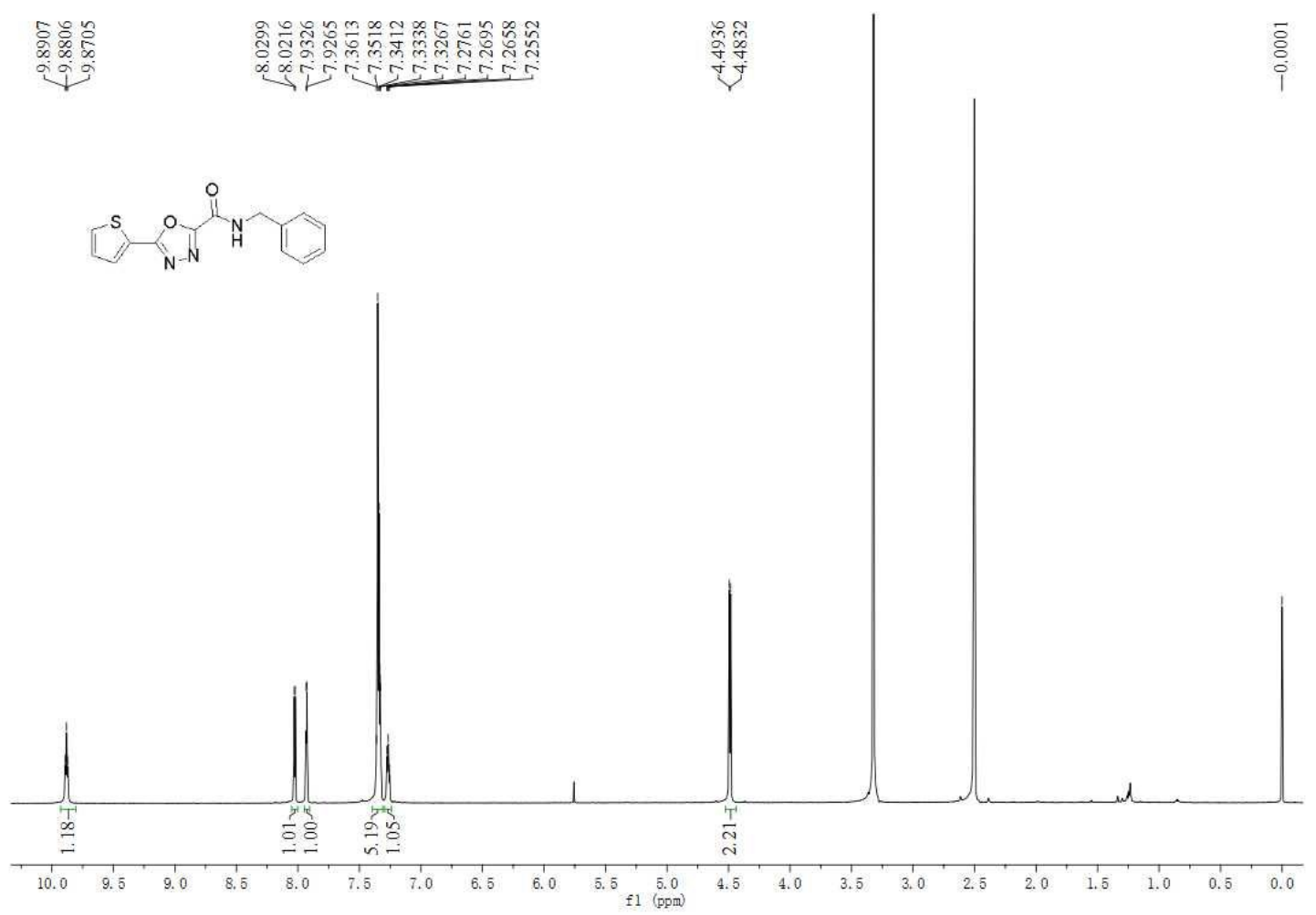

Figure S29. The ${ }^{1} \mathrm{H}$ NMR spectrum (600 MHz, DMSO- $\left.d_{6}\right)$ of compound $4 \mathbf{n}$
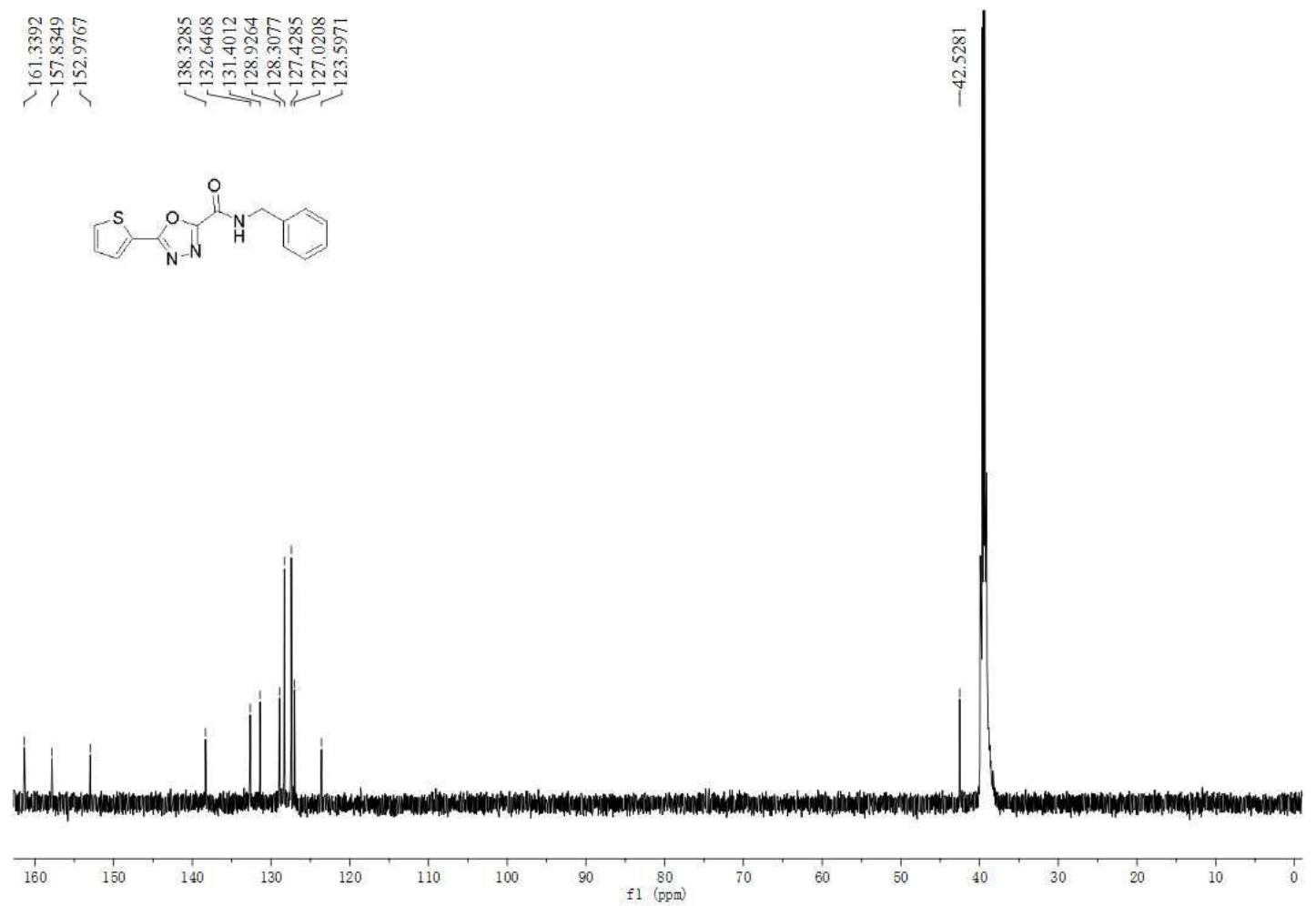

Figure S30. The ${ }^{13} \mathrm{C}$ NMR spectrum $\left(150 \mathrm{MHz}\right.$, DMSO- $\left.d_{6}\right)$ of compound $\mathbf{4 n}$ 


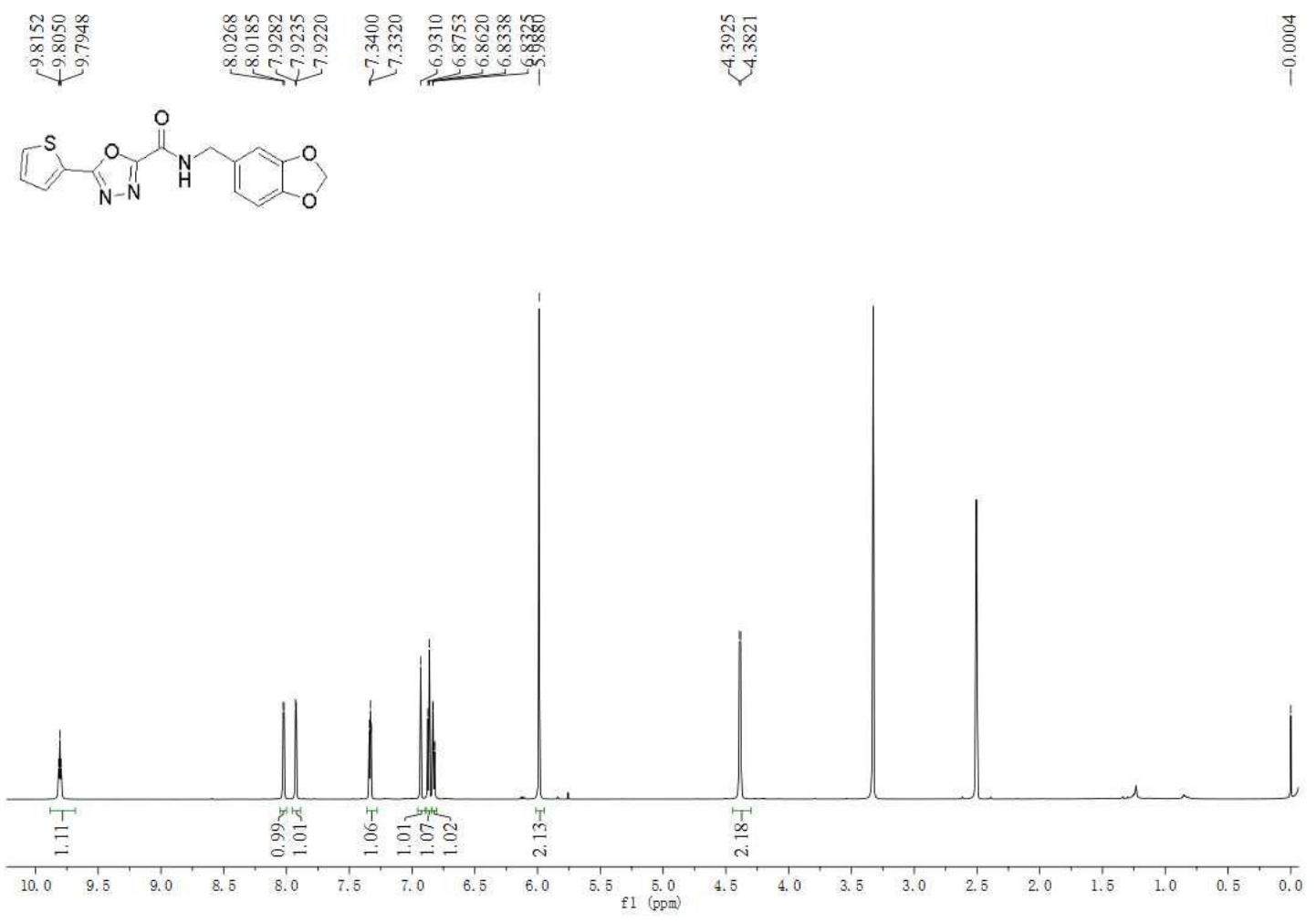

Figure S31. The ${ }^{1} \mathrm{H}$ NMR spectrum $\left(600 \mathrm{MHz}, \mathrm{DMSO}-d_{6}\right)$ of compound $4 \mathrm{o}$ 

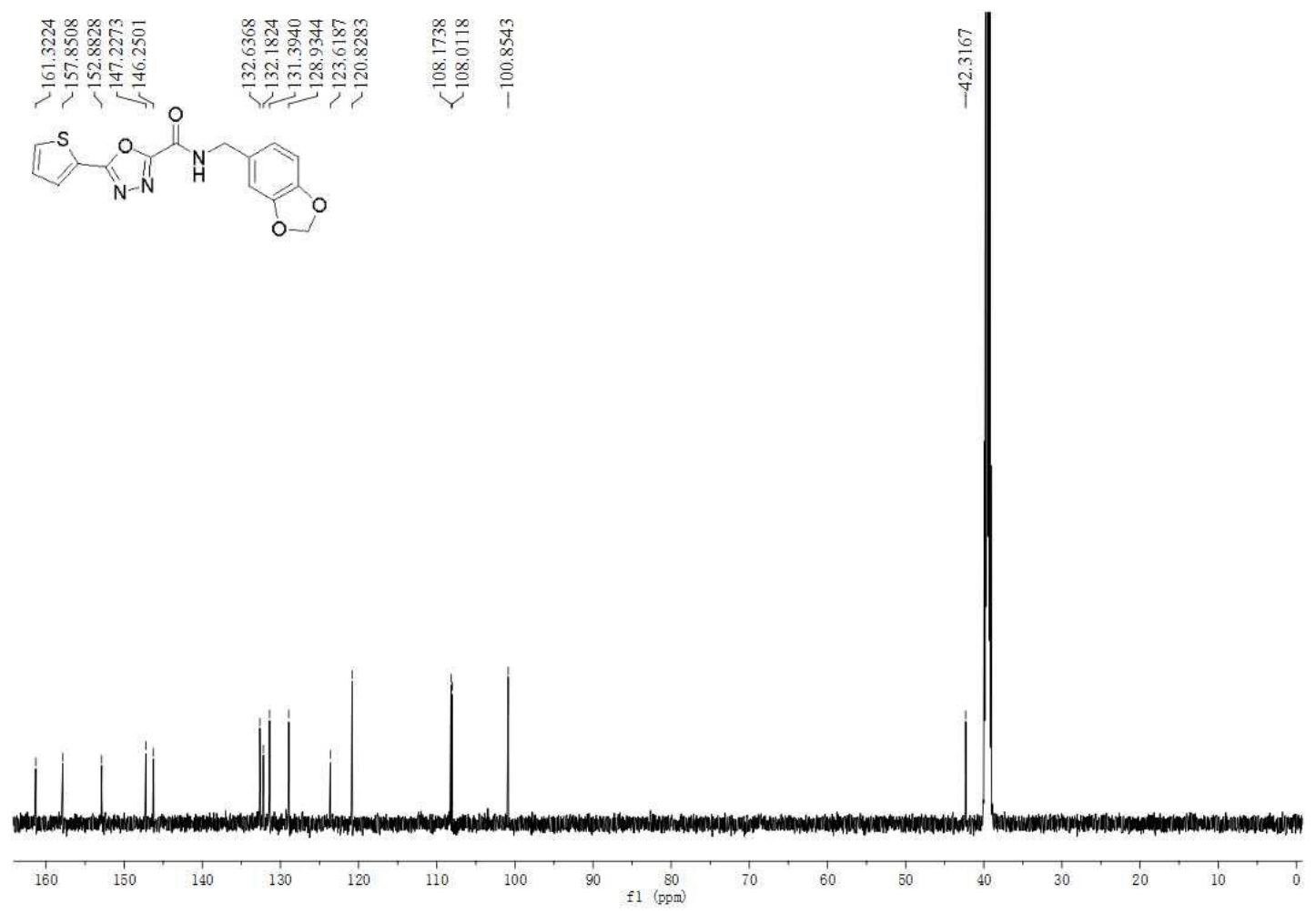

Figure S32. The ${ }^{13} \mathrm{C}$ NMR spectrum (150 MHz, DMSO- $\left.d_{6}\right)$ of compound 40

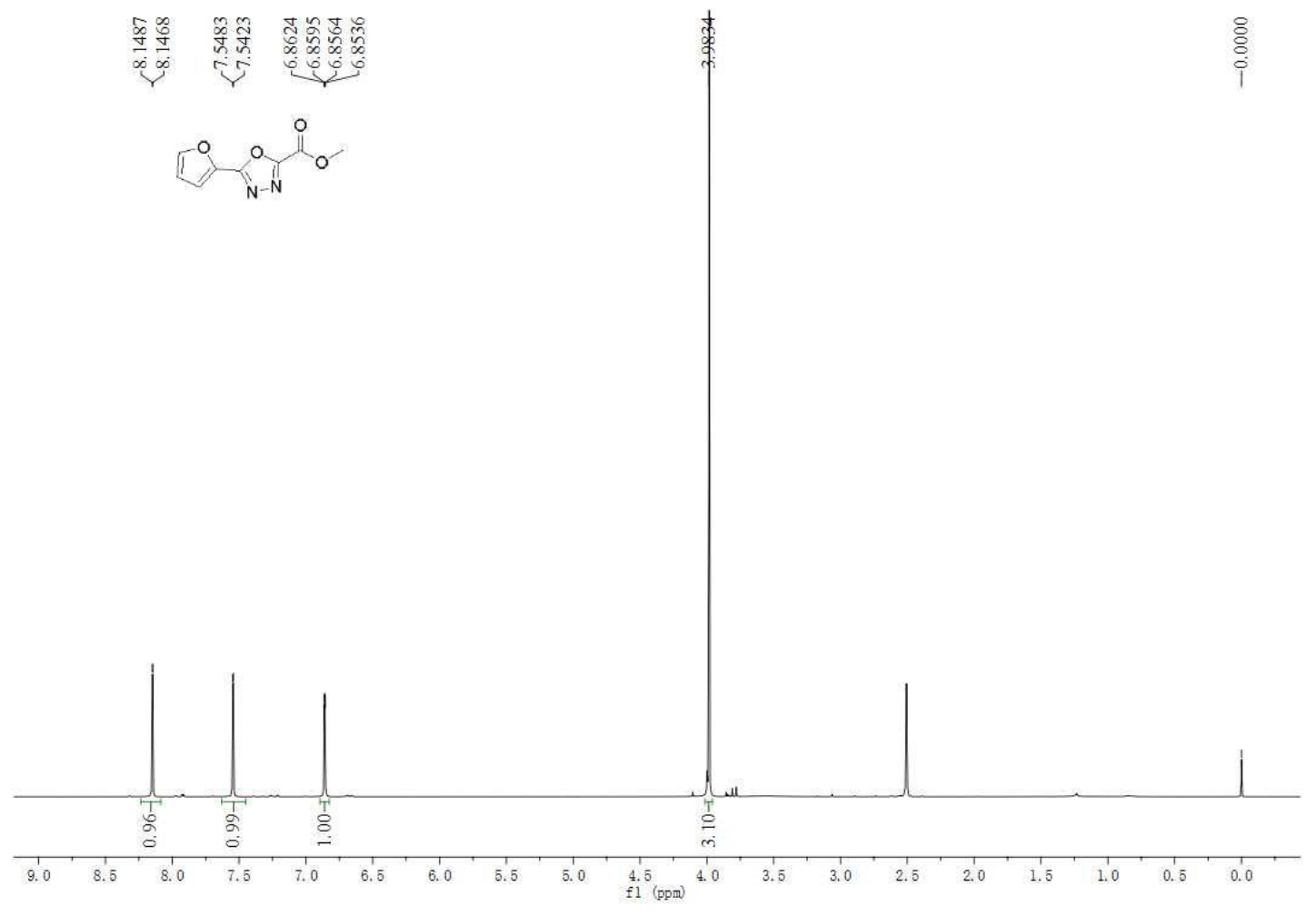

Figure S33. The ${ }^{1} \mathrm{H}$ NMR spectrum (600 MHz, DMSO- $d_{6}$ ) of intermediate $3 \mathbf{b}$ 


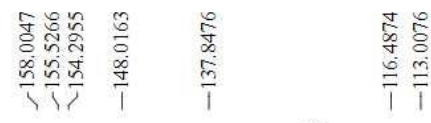

$$
\begin{aligned}
& \text { II) } \sum_{n-N}^{\infty} e^{\pi}
\end{aligned}
$$

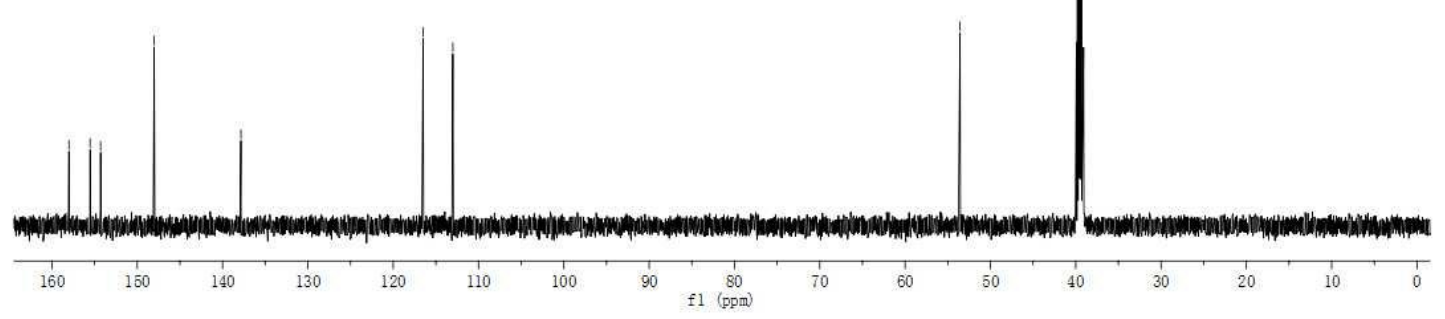

Figure S34. The ${ }^{13} \mathrm{C}$ NMR spectrum (150 MHz, DMSO- $\left.d_{6}\right)$ of intermediate $3 \mathbf{b}$

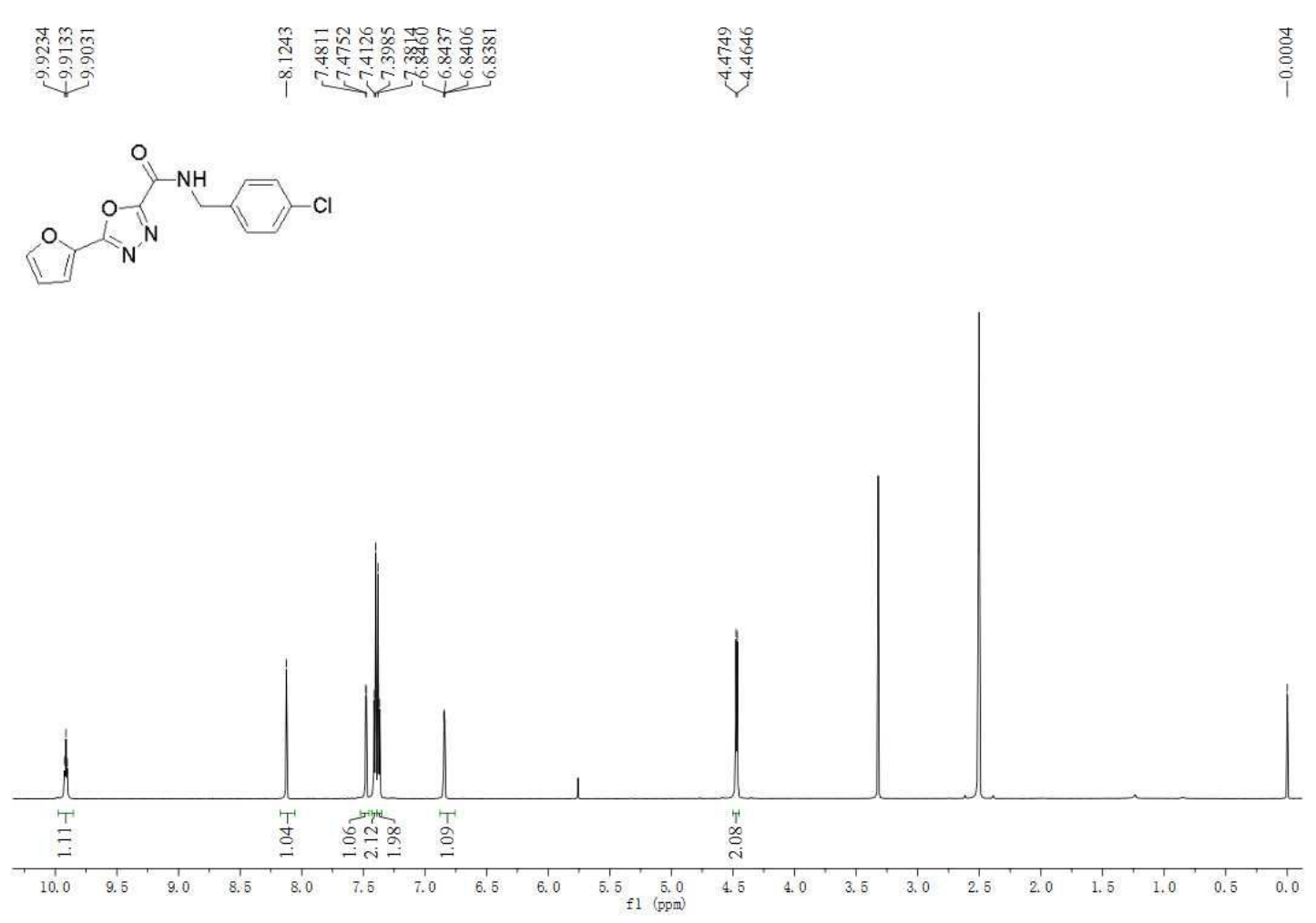


Figure S35. The ${ }^{1} \mathrm{H}$ NMR spectrum ( $600 \mathrm{MHz}$, DMSO- $d_{6}$ ) of compound $\mathbf{5 a}$
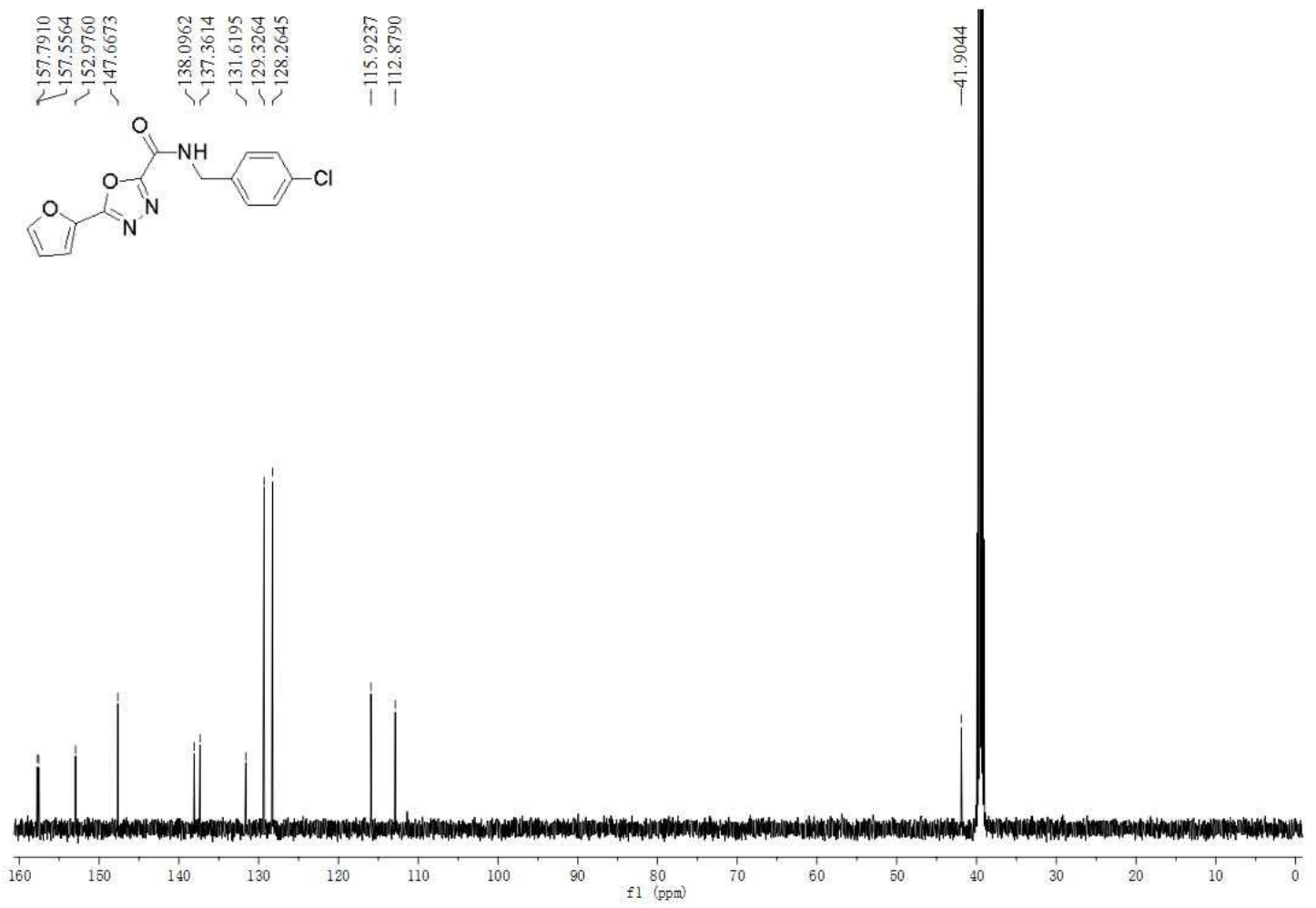

Figure S36. The ${ }^{13} \mathrm{C}$ NMR spectrum $\left(150 \mathrm{MHz}\right.$, DMSO- $\left.d_{6}\right)$ of compound 5a 


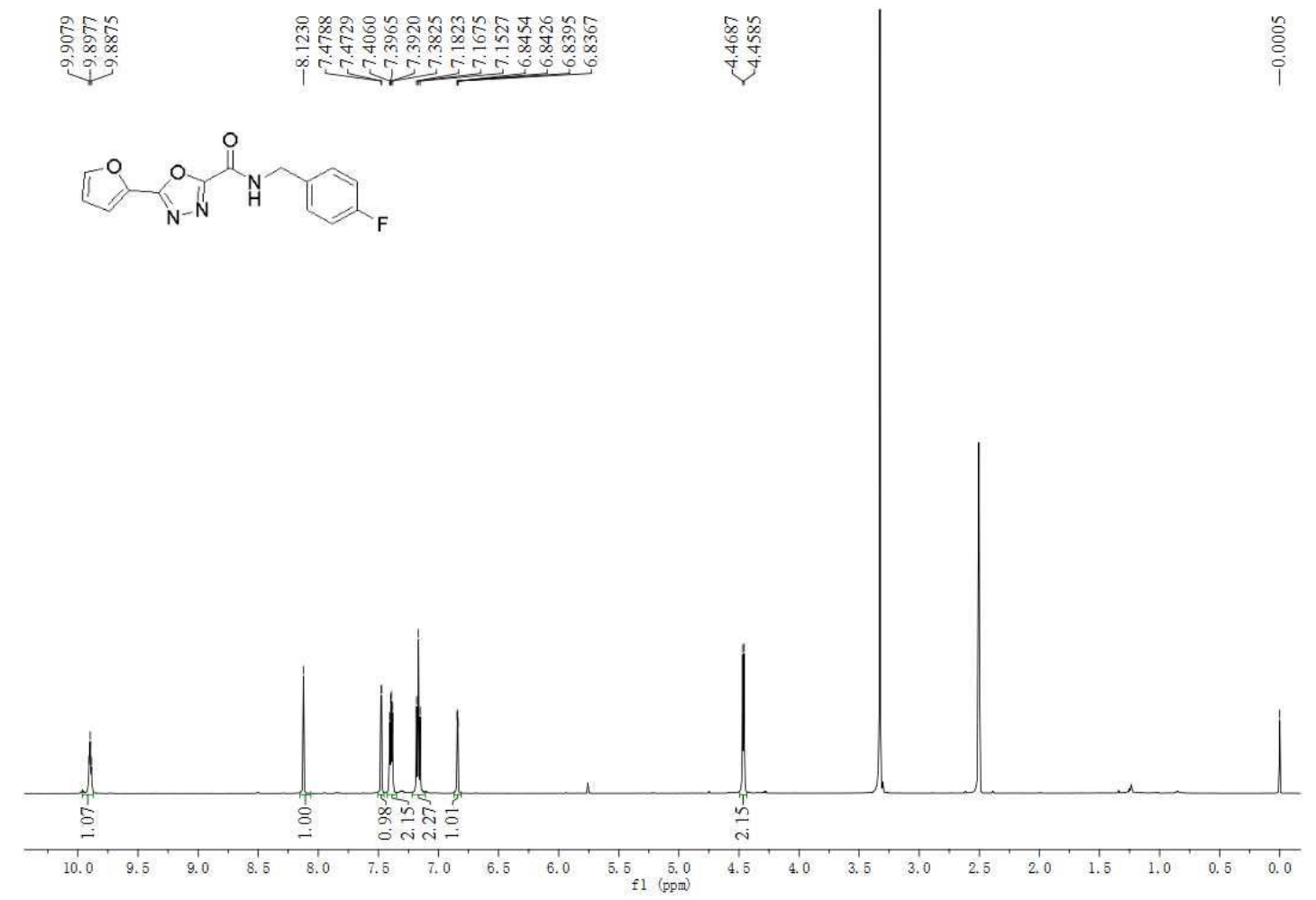

Figure S37. The ${ }^{1} \mathrm{H}$ NMR spectrum (600 MHz, DMSO- $d_{6}$ ) of compound $\mathbf{5 b}$ 

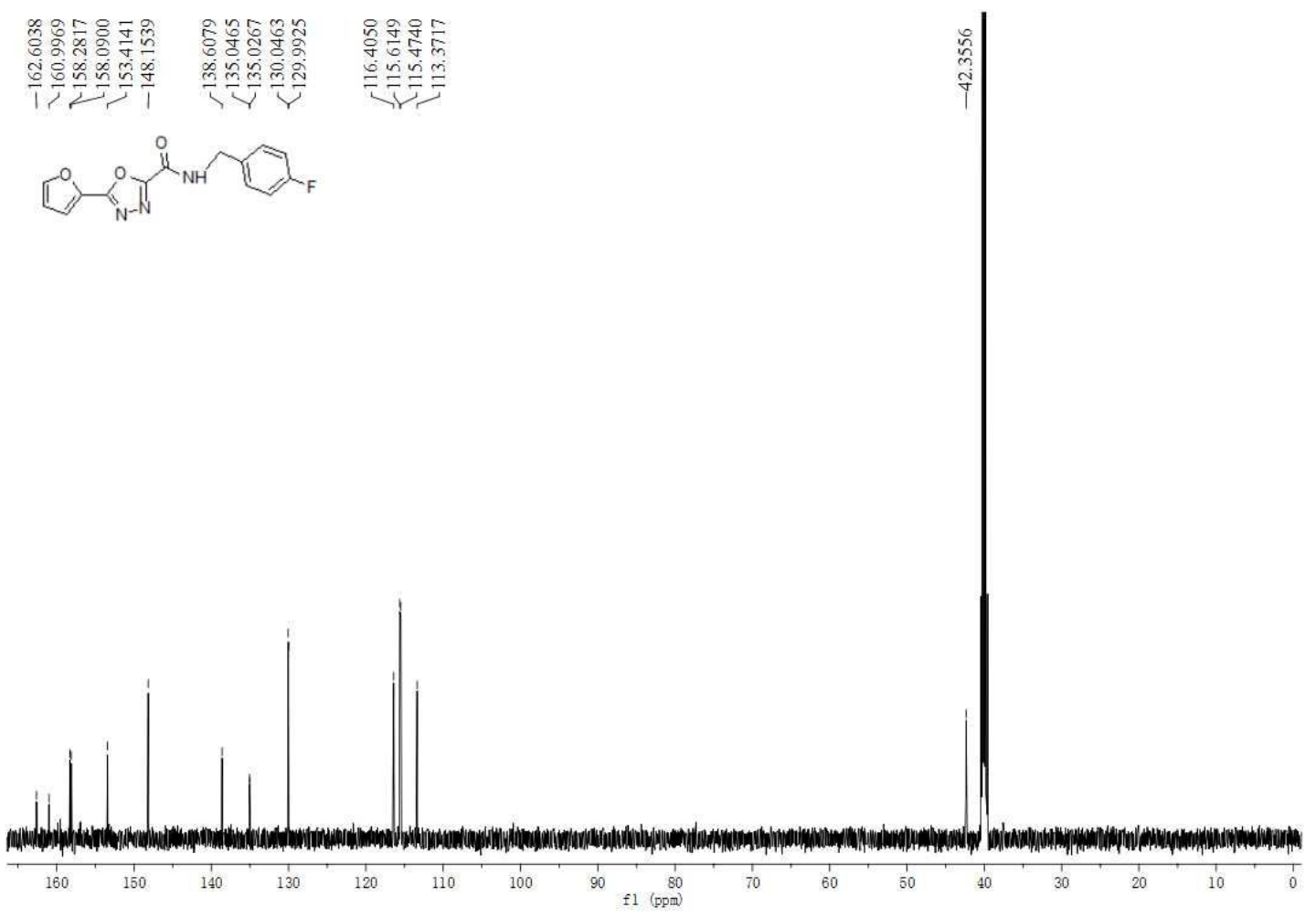

Figure S38. The ${ }^{13} \mathrm{C}$ NMR spectrum $\left(150 \mathrm{MHz}, \mathrm{DMSO}-d_{6}\right)$ of compound $\mathbf{5 b}$

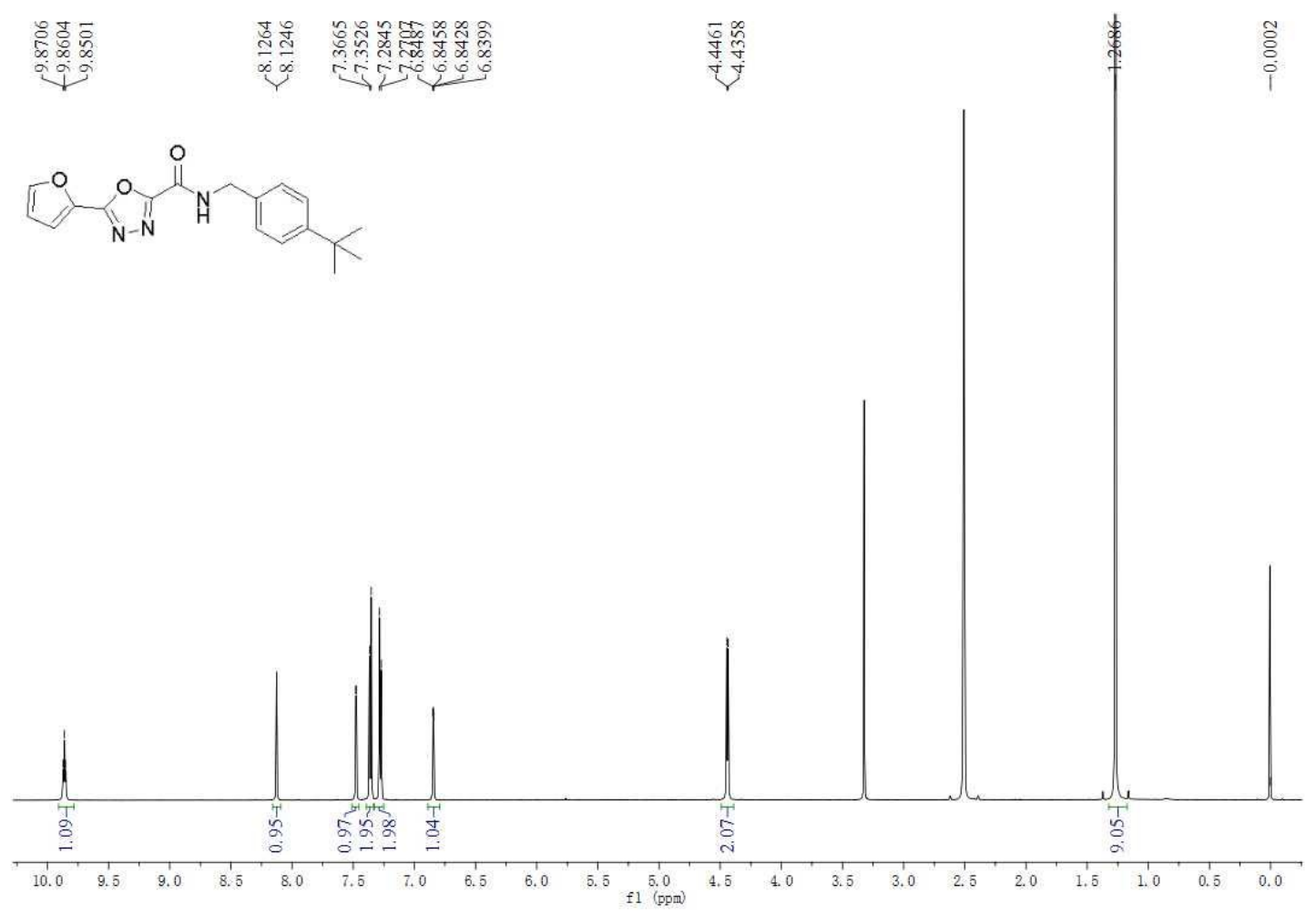

Figure S39. The ${ }^{1} \mathrm{H}$ NMR spectrum $\left(600 \mathrm{MHz}, \mathrm{DMSO}-d_{6}\right)$ of compound $\mathbf{5 c}$ 

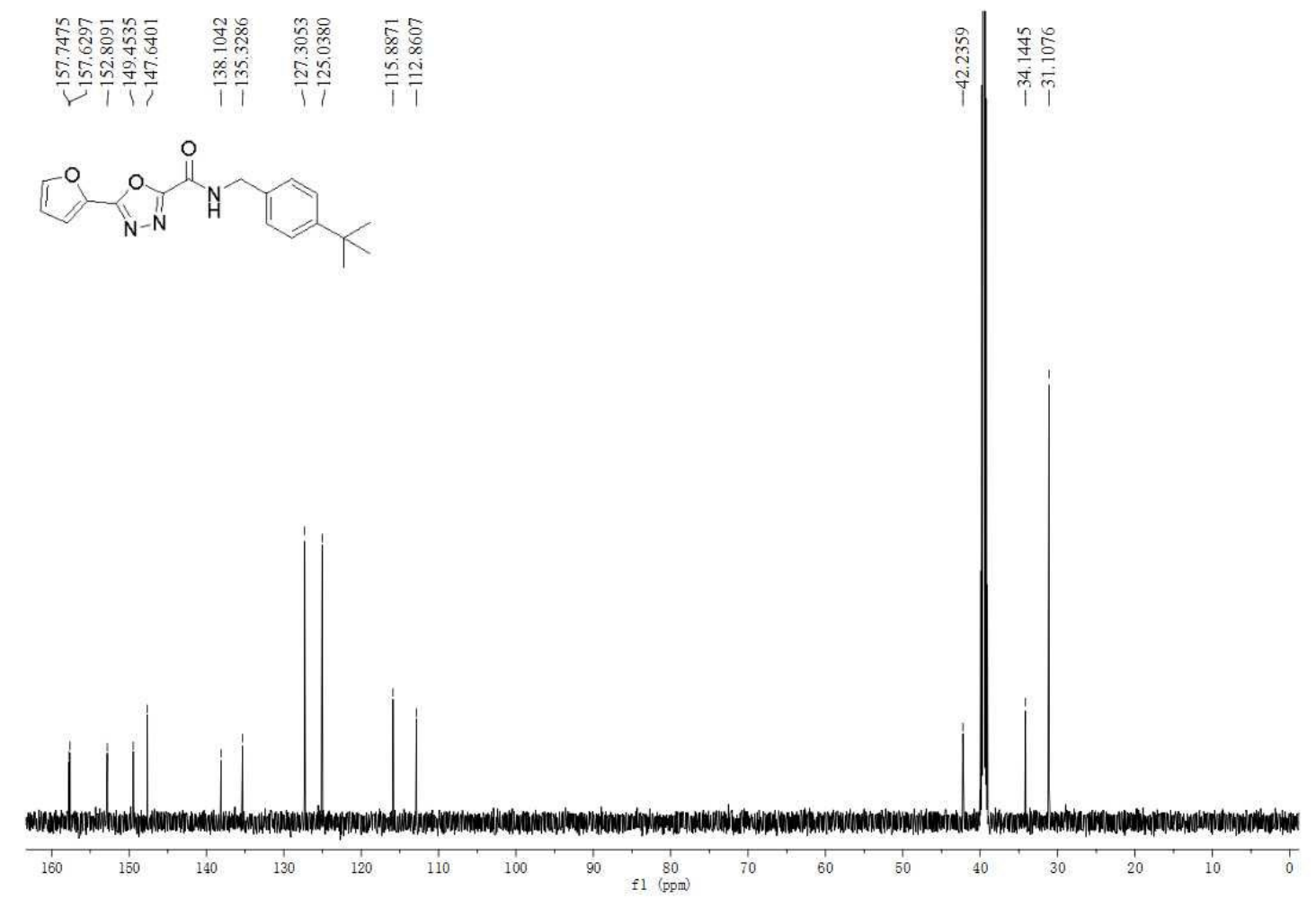

Figure S40. The ${ }^{13} \mathrm{C}$ NMR spectrum (150MHz, DMSO- $d_{6}$ ) of compound $\mathbf{5 c}$

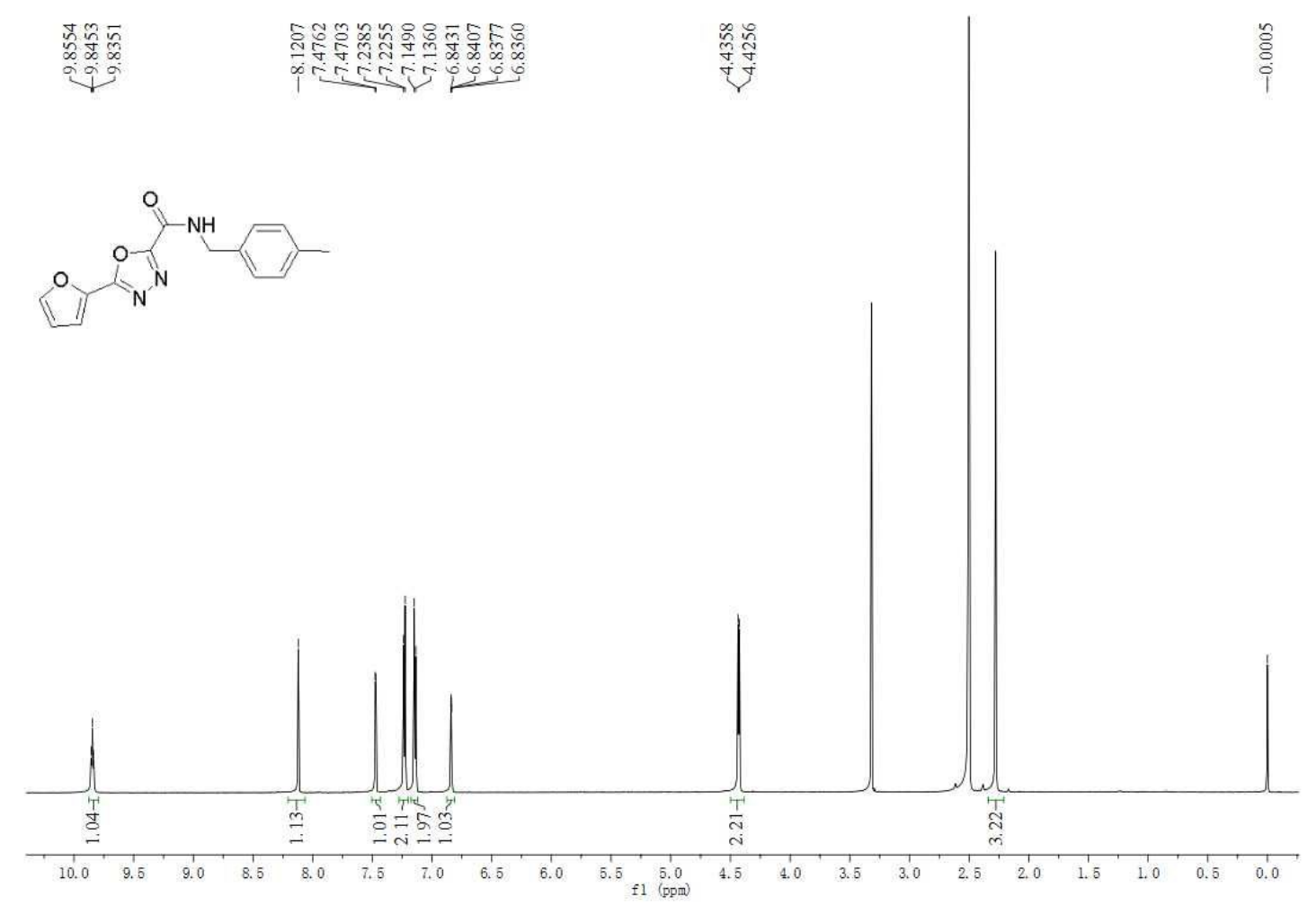

Figure S41. The ${ }^{1} \mathrm{H}$ NMR spectrum (600 MHz, DMSO- $d_{6}$ ) of compound 5d 


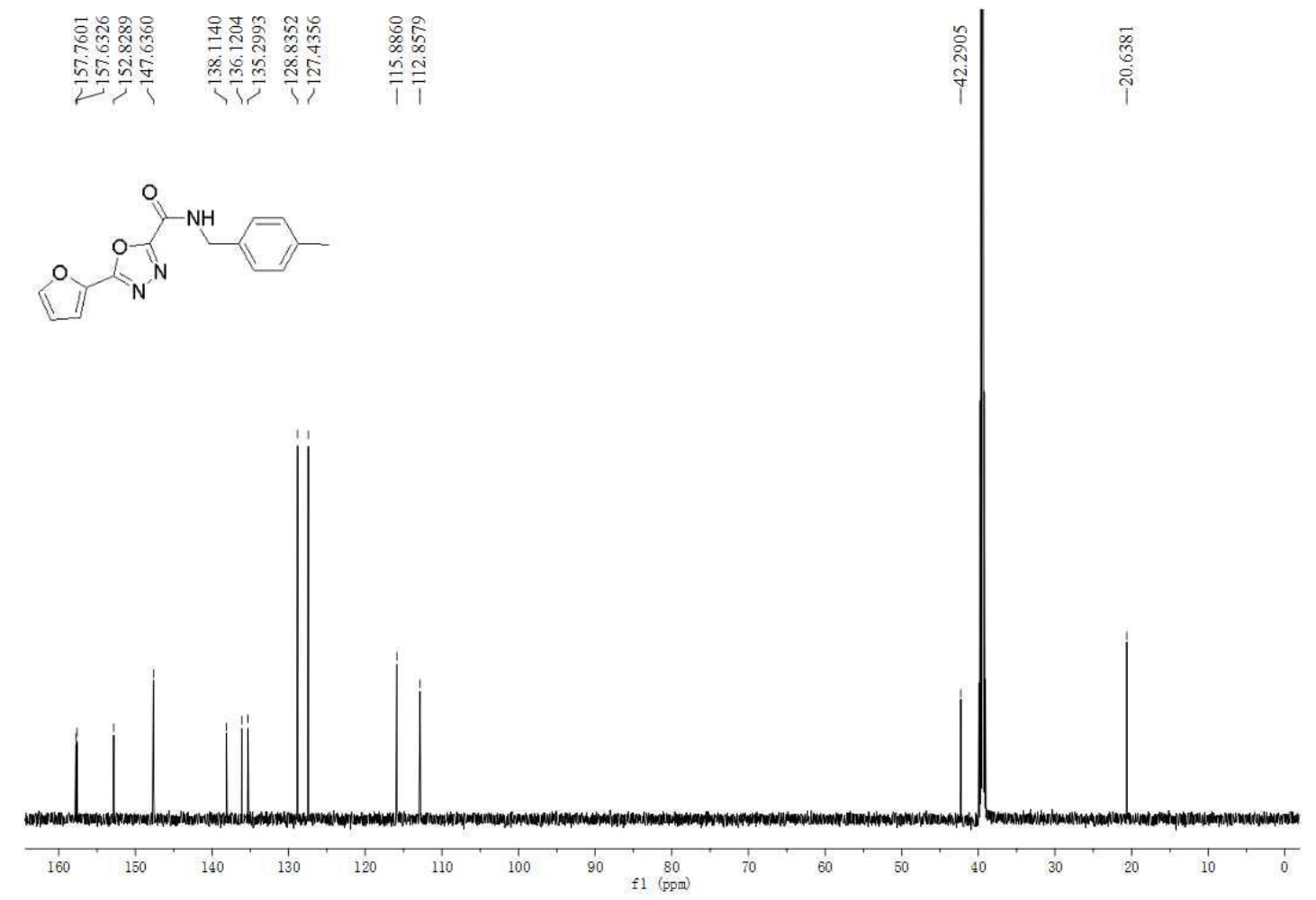

Figure S42. The ${ }^{13} \mathrm{C}$ NMR spectrum $\left(150 \mathrm{MHz}, \mathrm{DMSO}-d_{6}\right)$ of compound $\mathbf{5 d}$ 


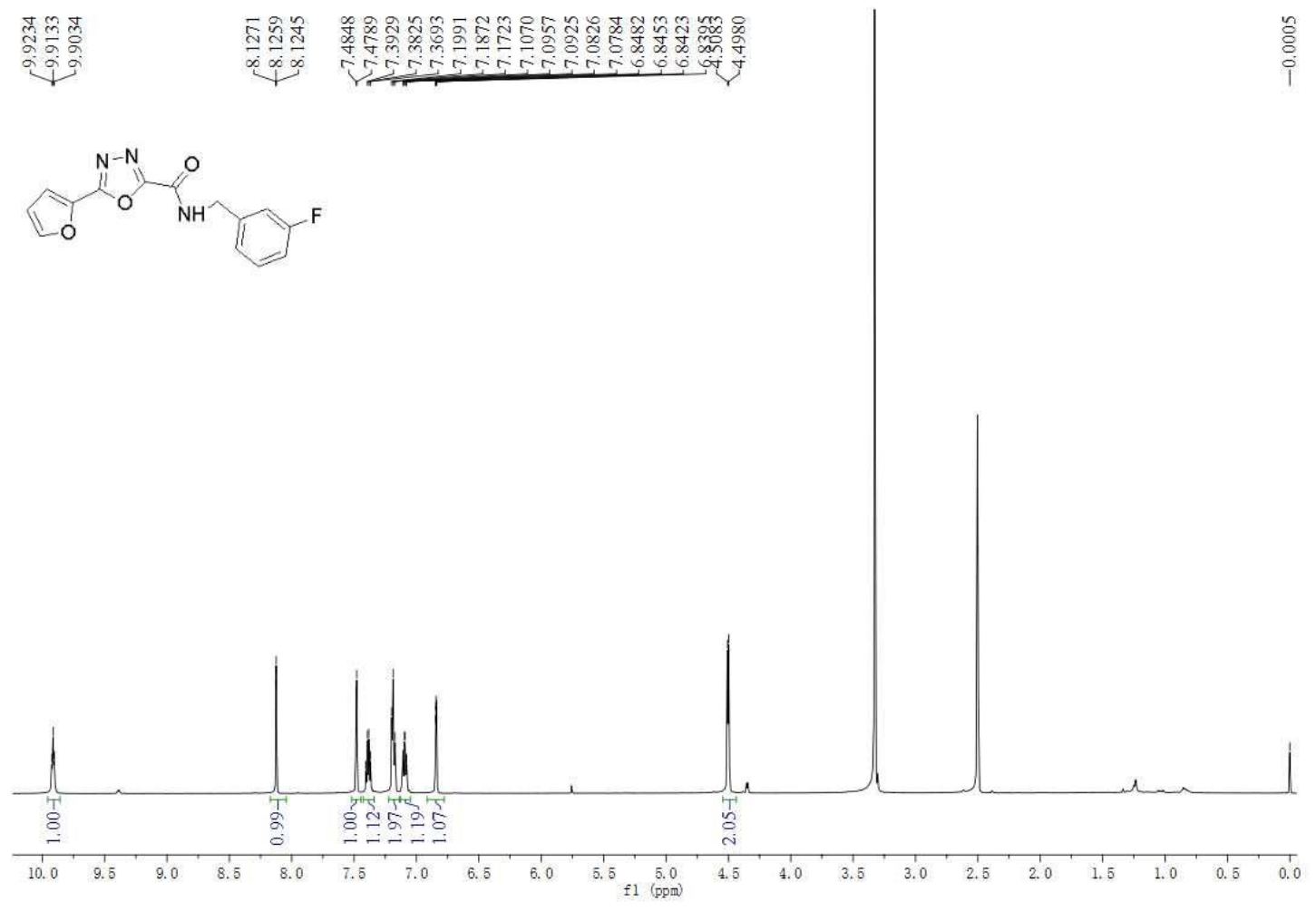

Figure S43. The ${ }^{1} \mathrm{H}$ NMR spectrum (600 MHz, DMSO- $d_{6}$ ) of compound $5 \mathbf{e}$ 


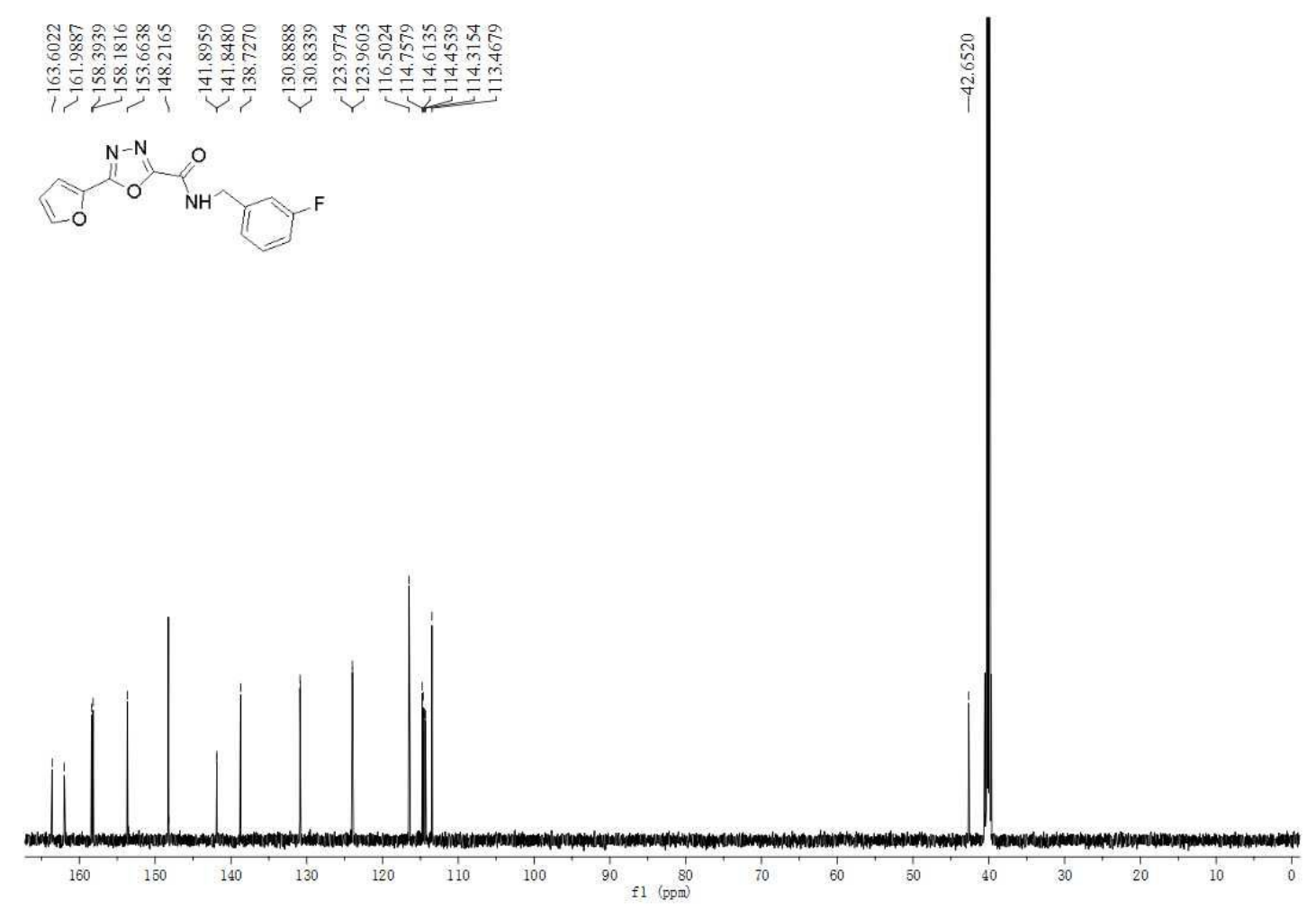

Figure S44. The ${ }^{13} \mathrm{C}$ NMR spectrum $\left(150 \mathrm{MHz}, \mathrm{DMSO}-d_{6}\right)$ of compound $\mathbf{5 e}$
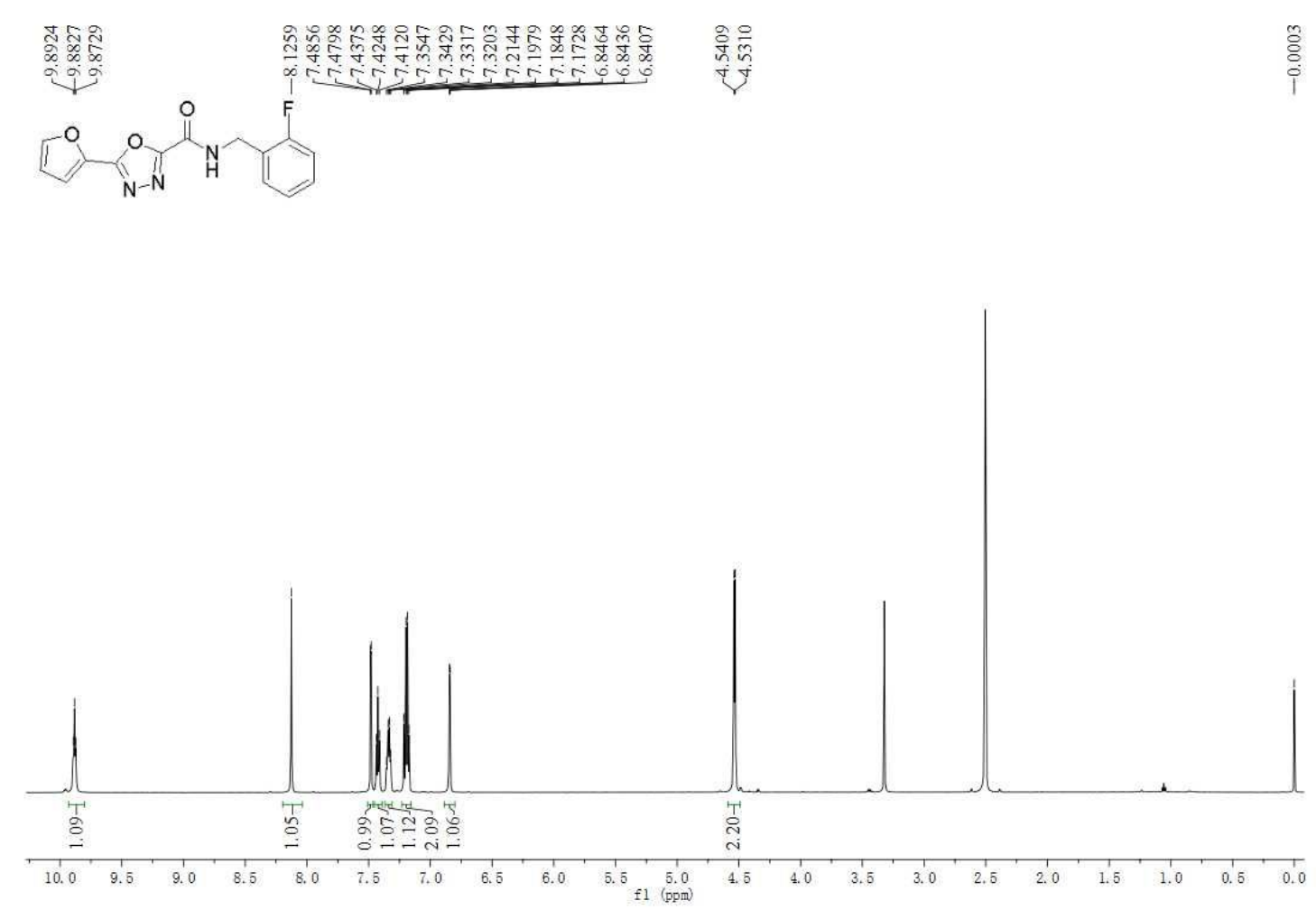

Figure S45. The ${ }^{1} \mathrm{H}$ NMR spectrum $\left(600 \mathrm{MHz}, \mathrm{DMSO}-d_{6}\right)$ of compound $\mathbf{5 f}$ 

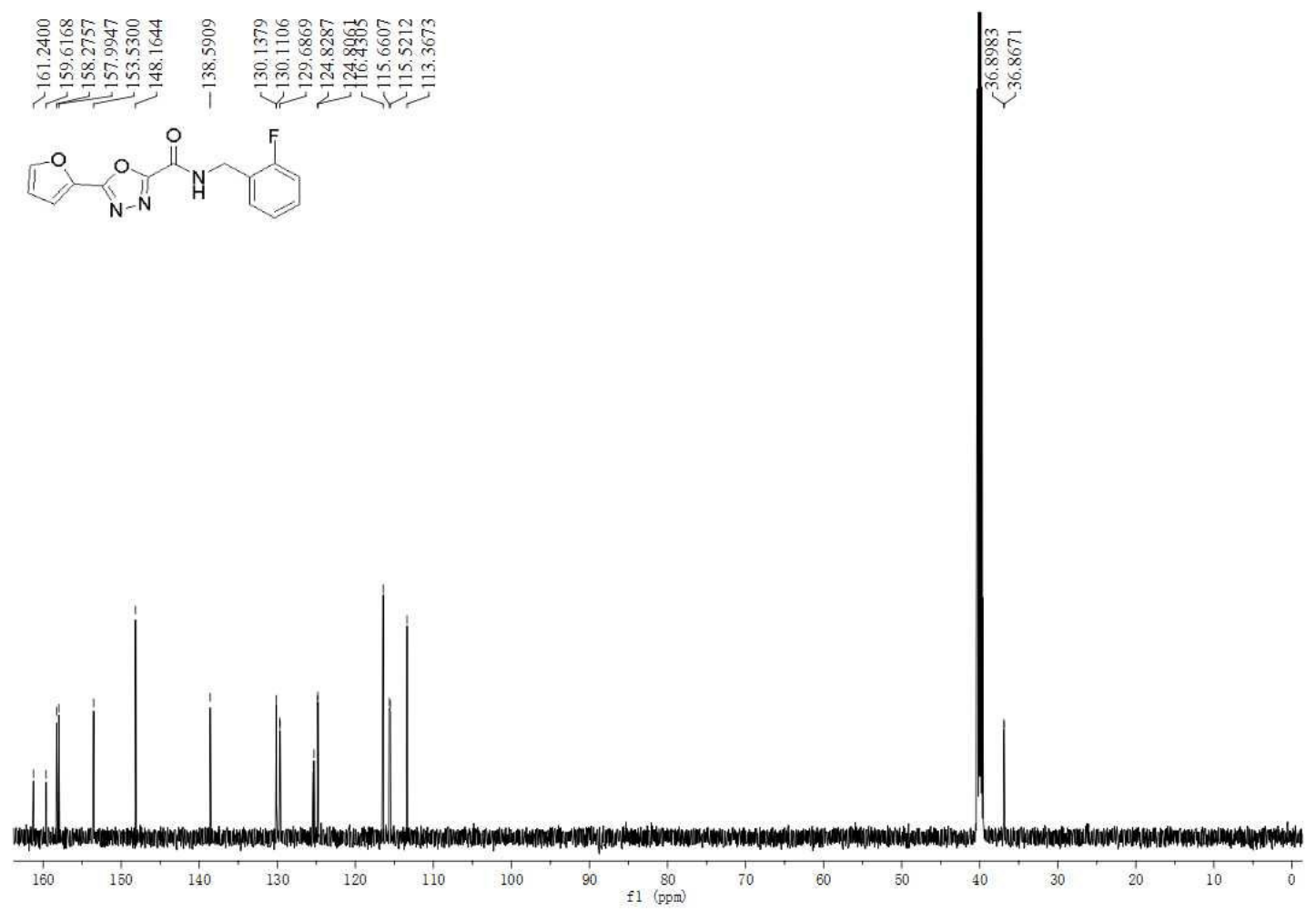

Figure S46. The ${ }^{13} \mathrm{CNMR}$ spectrum (150 MHz, DMSO- $\left.d_{6}\right)$ of compound $\mathbf{5 f}$

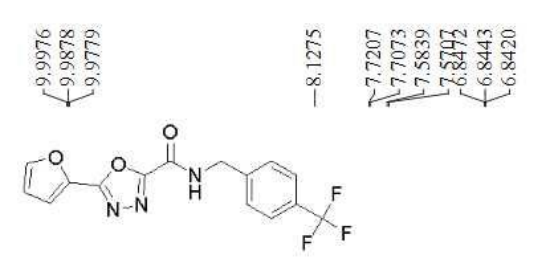

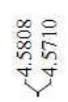$$
\begin{aligned}
& \text { å } \\
& \vdots \\
& 0 \\
& i
\end{aligned}
$$

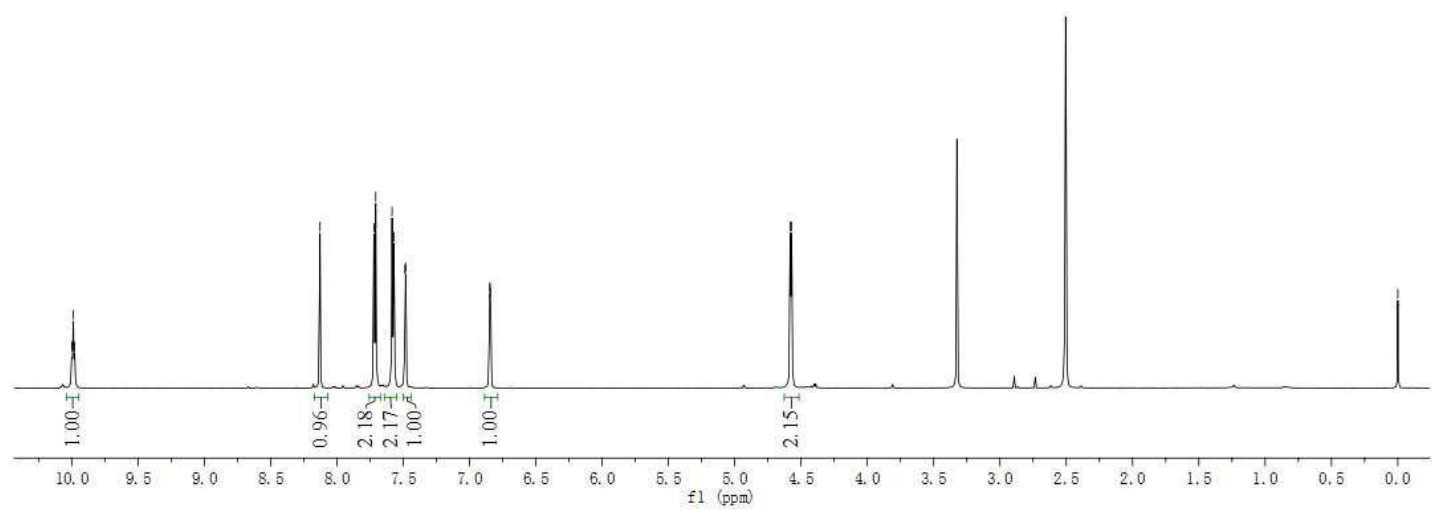

Figure S47. The ${ }^{1} \mathrm{H}$ NMR spectrum $\left(600 \mathrm{MHz}, \mathrm{DMSO}-d_{6}\right)$ of compound $\mathbf{5 g}$ 


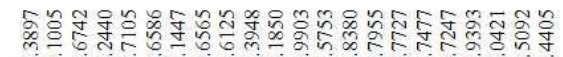

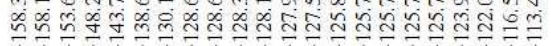
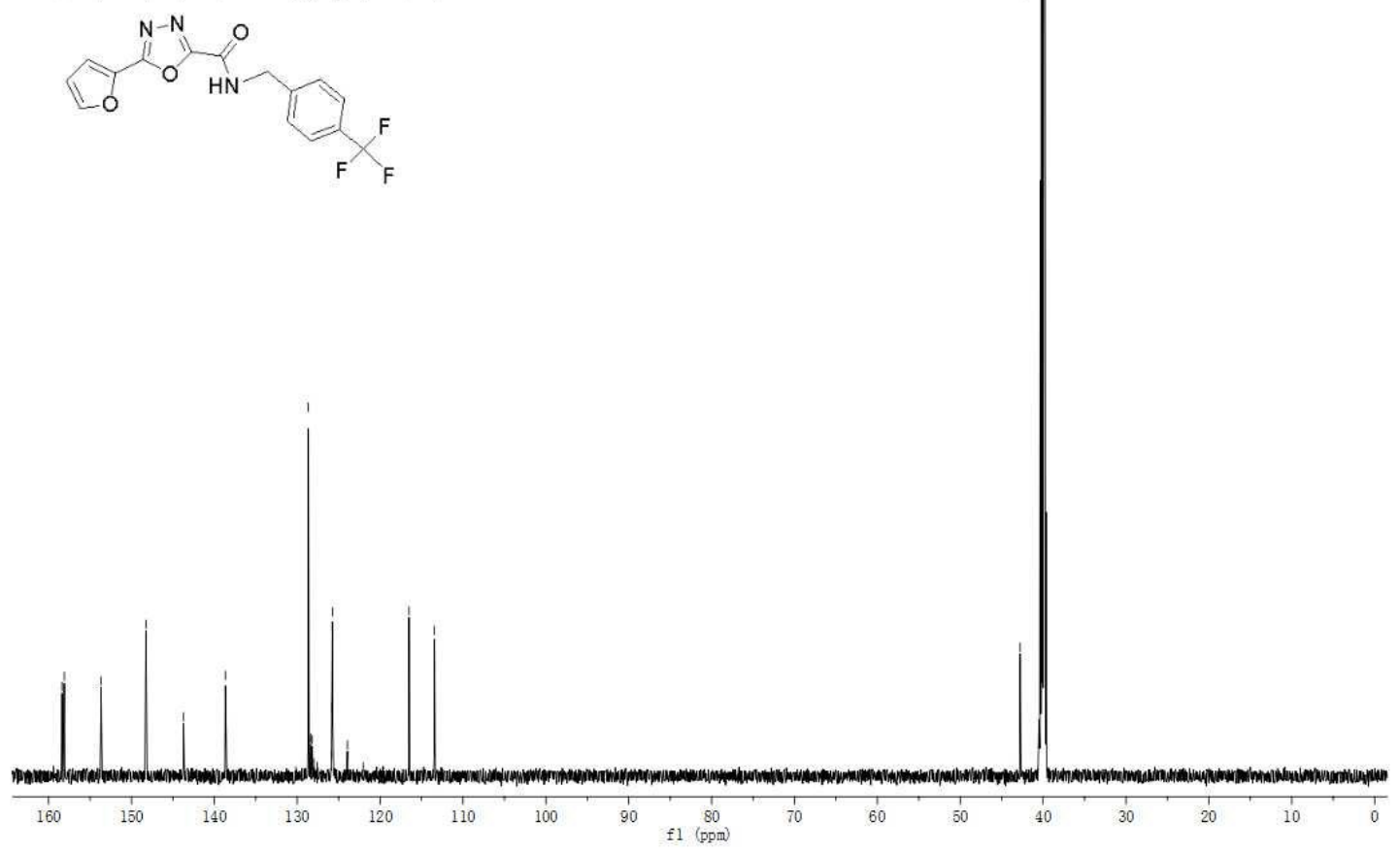

Figure S48. The ${ }^{13} \mathrm{CNMR}$ spectrum (150 MHz, DMSO- $d_{6}$ ) of compound $\mathbf{5 g}$

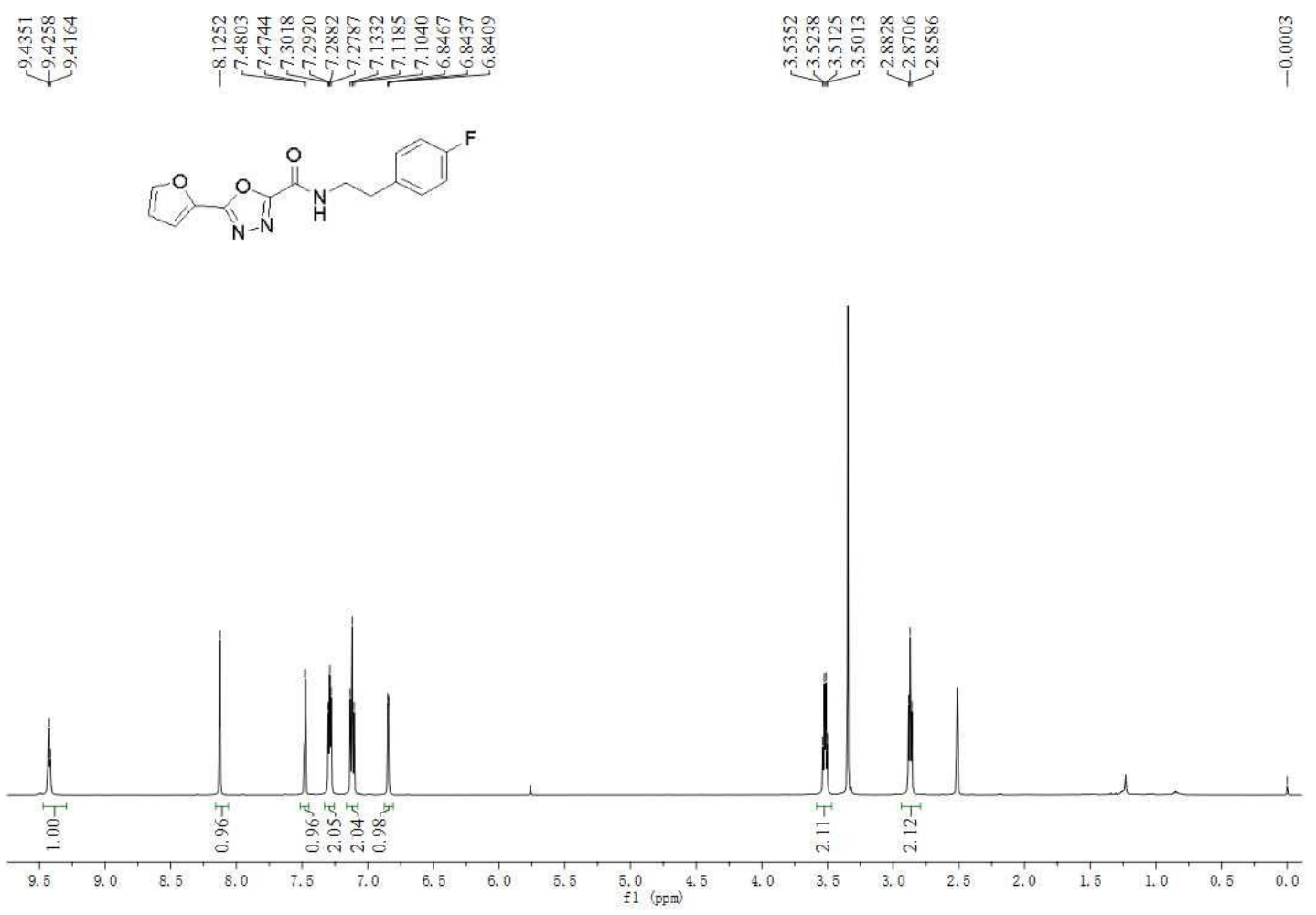

Figure S49. The ${ }^{1} \mathrm{H}$ NMR spectrum ( $600 \mathrm{MHz}$, DMSO- $\left.d_{6}\right)$ of compound $\mathbf{5 h}$ 


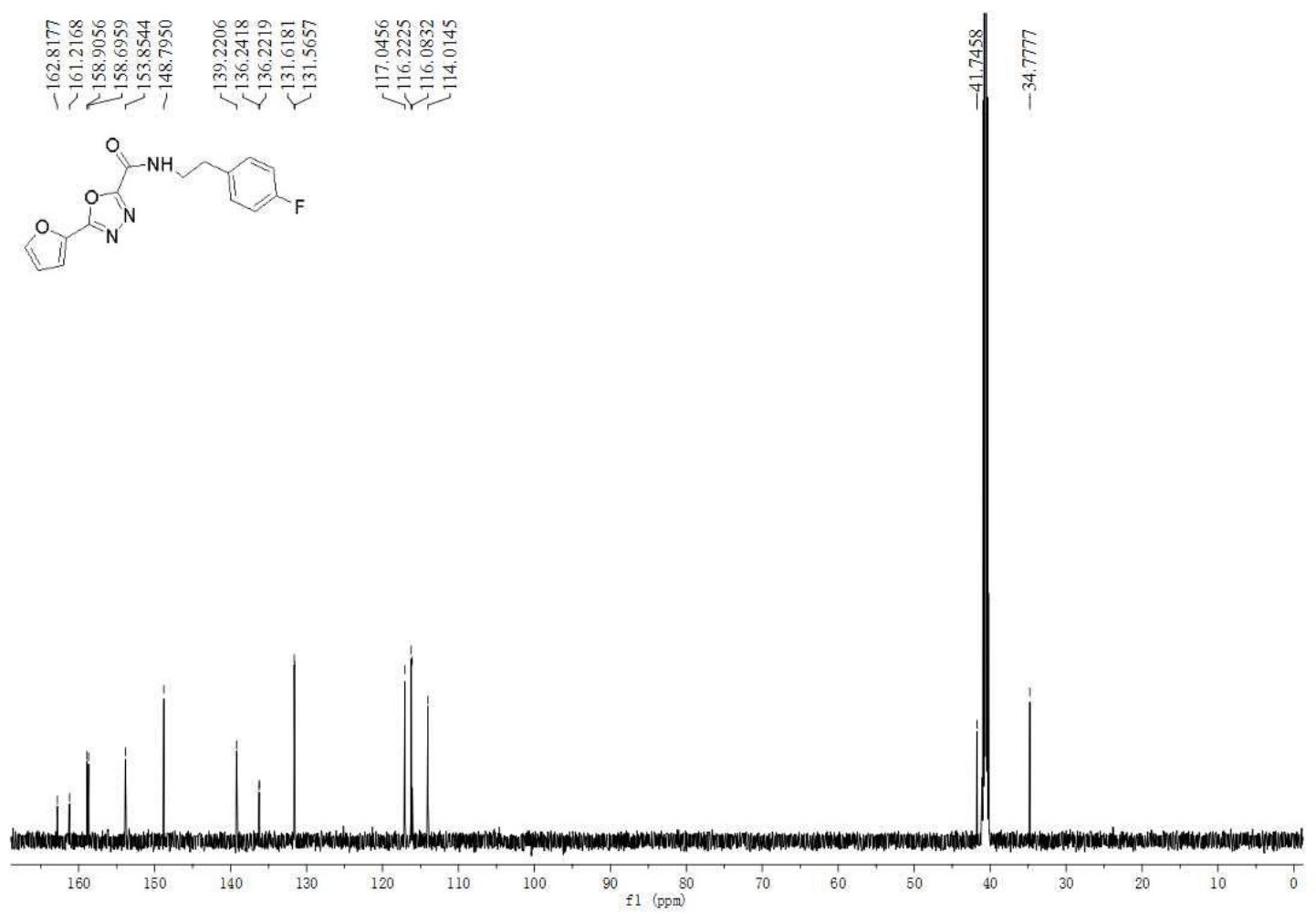

Figure S50. The ${ }^{13} \mathrm{C}$ NMR spectrum (150 MHz, DMSO- $d_{6}$ ) of compound $\mathbf{5 h}$

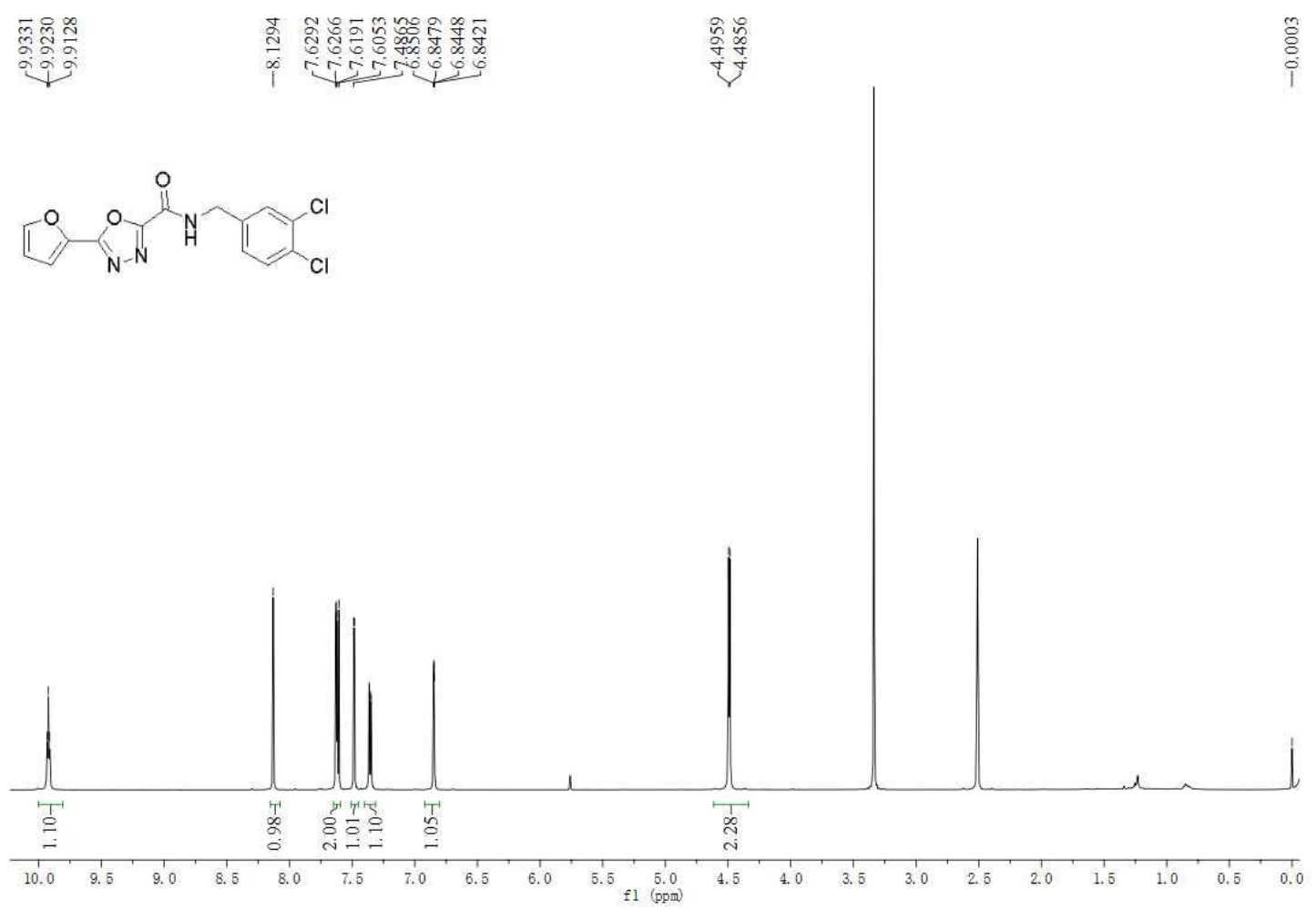

Figure S51. The ${ }^{1} \mathrm{H}$ NMR spectrum $\left(600 \mathrm{MHz}, \mathrm{DMSO}-d_{6}\right)$ of compound $\mathbf{5 i}$ 


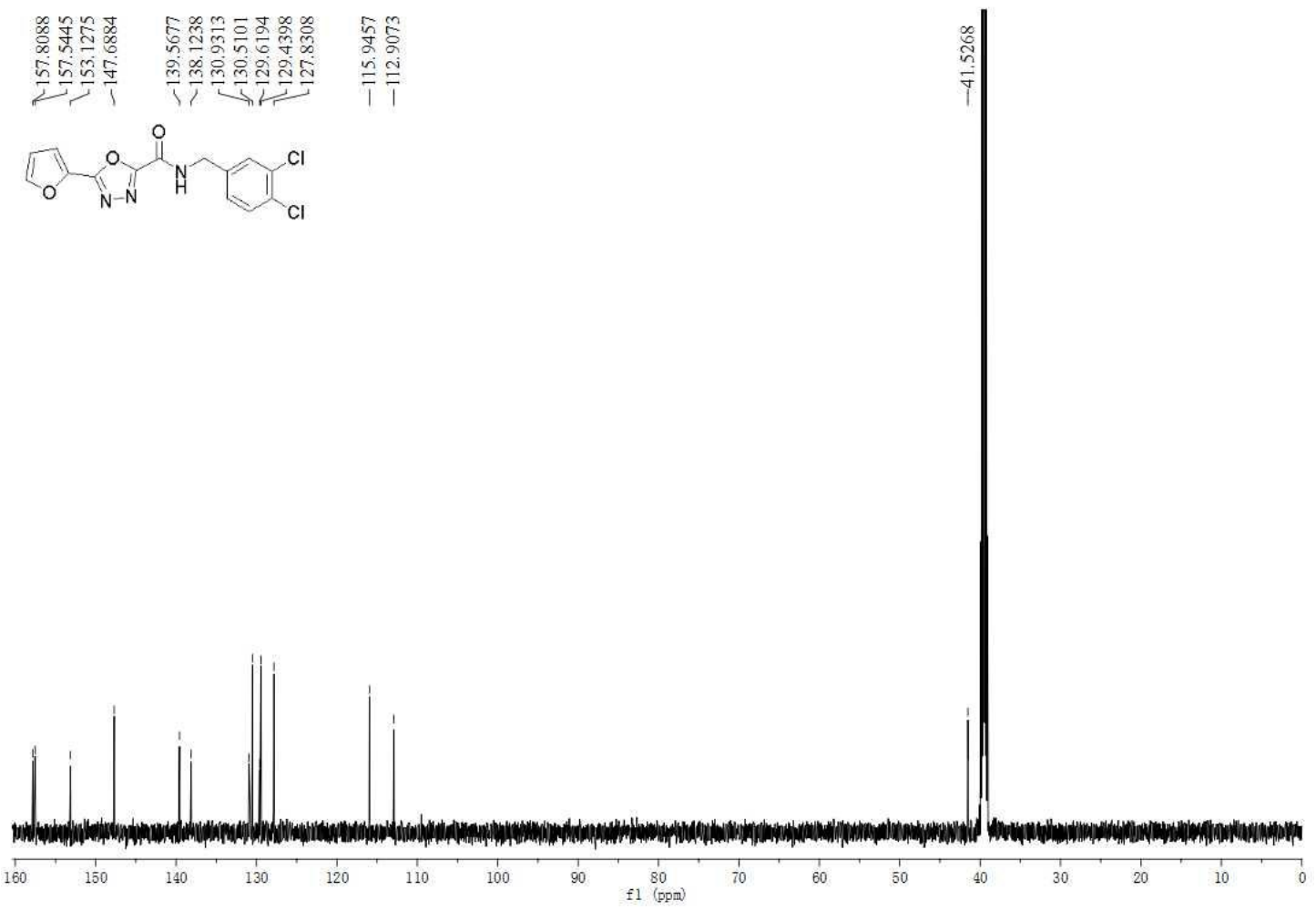

Figure S52. The ${ }^{13} \mathrm{C}$ NMR spectrum $\left(150 \mathrm{MHz}, \mathrm{DMSO}-d_{6}\right)$ of compound $\mathbf{5 i}$ 


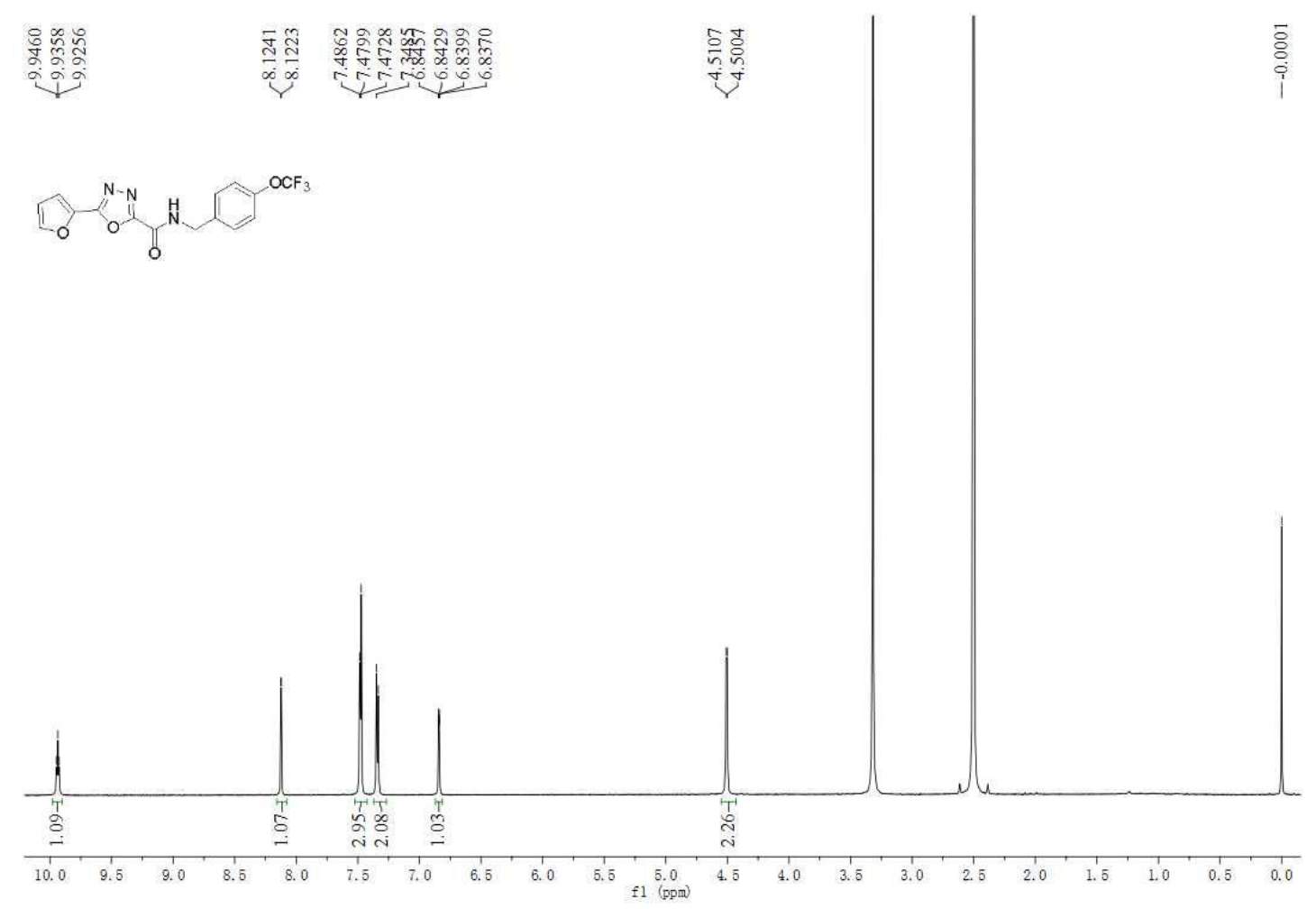

Figure S53. The ${ }^{1} \mathrm{H}$ NMR spectrum (600 MHz, DMSO- $\left.d_{6}\right)$ of compound $\mathbf{5 j}$
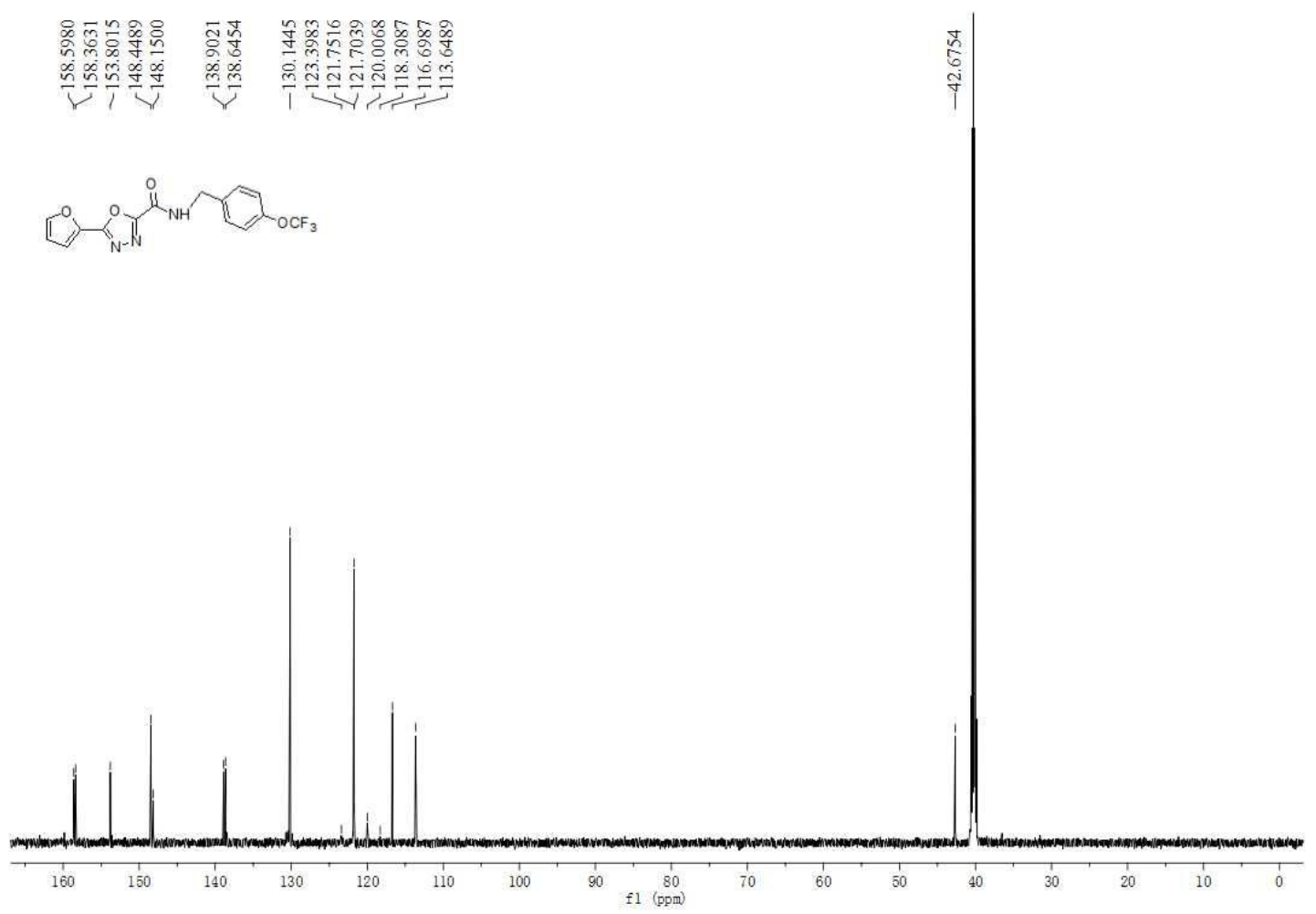

Figure S54. The ${ }^{13} \mathrm{CNMR}$ spectrum $\left(600 \mathrm{MHz}, \mathrm{DMSO}-d_{6}\right)$ of compound $\mathbf{5 j}$ 


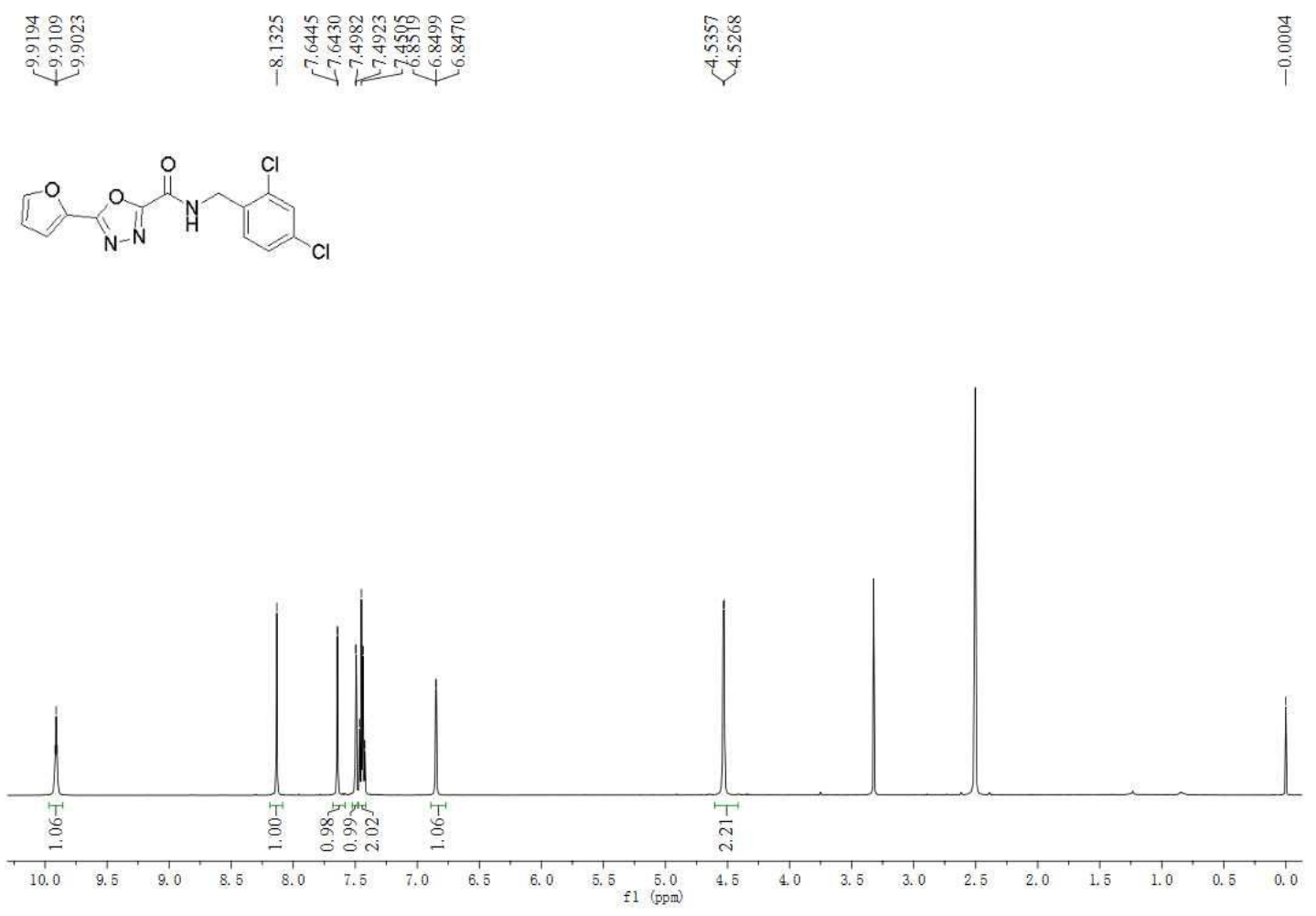

Figure S55. The ${ }^{1} \mathrm{H}$ NMR spectrum (600 MHz, DMSO- $\left.d_{6}\right)$ of compound $\mathbf{5 k}$ 

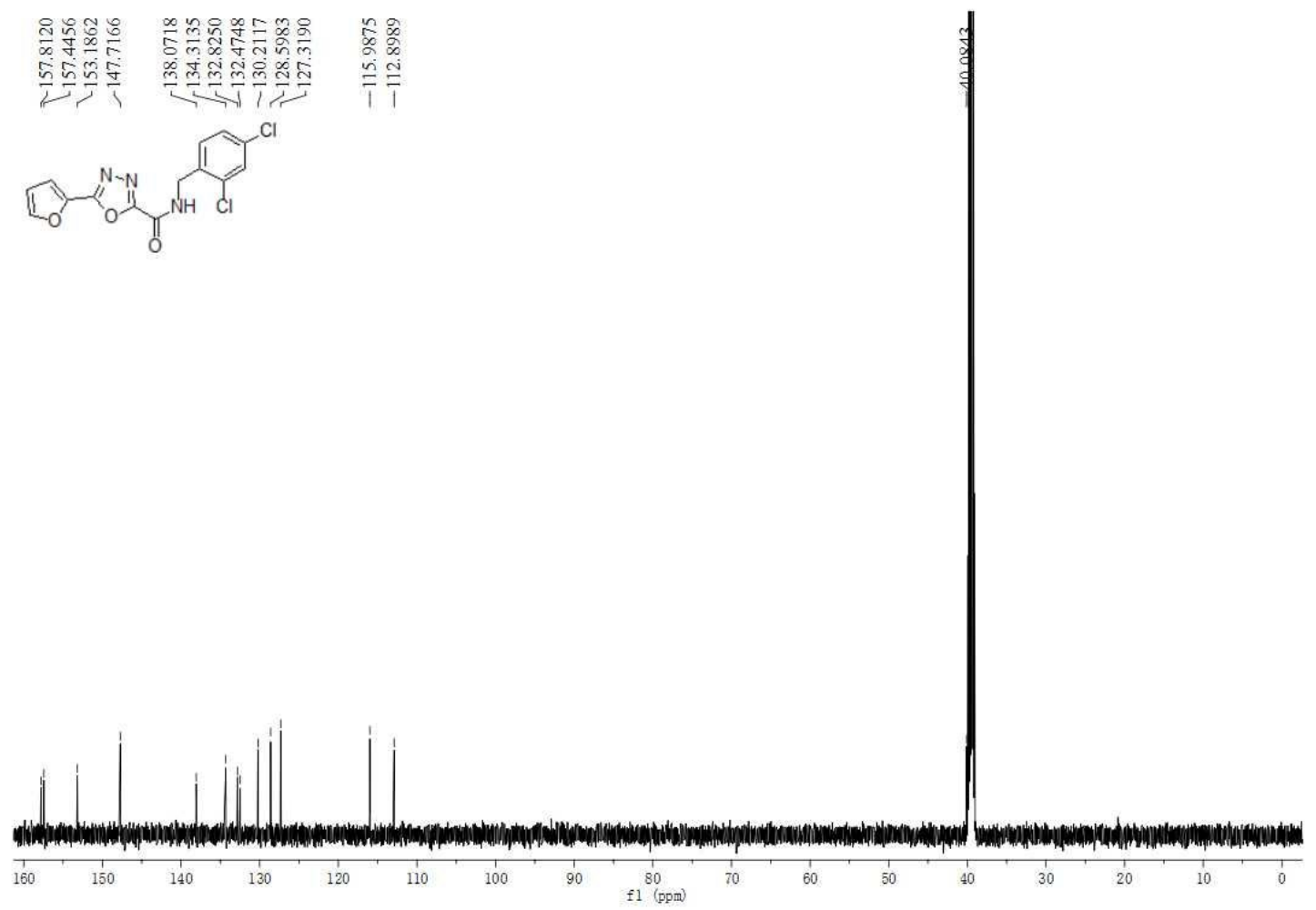

Figure S56. The ${ }^{13} \mathrm{CNMR}$ spectrum (150 MHz, DMSO- $d_{6}$ ) of compound $\mathbf{5 k}$
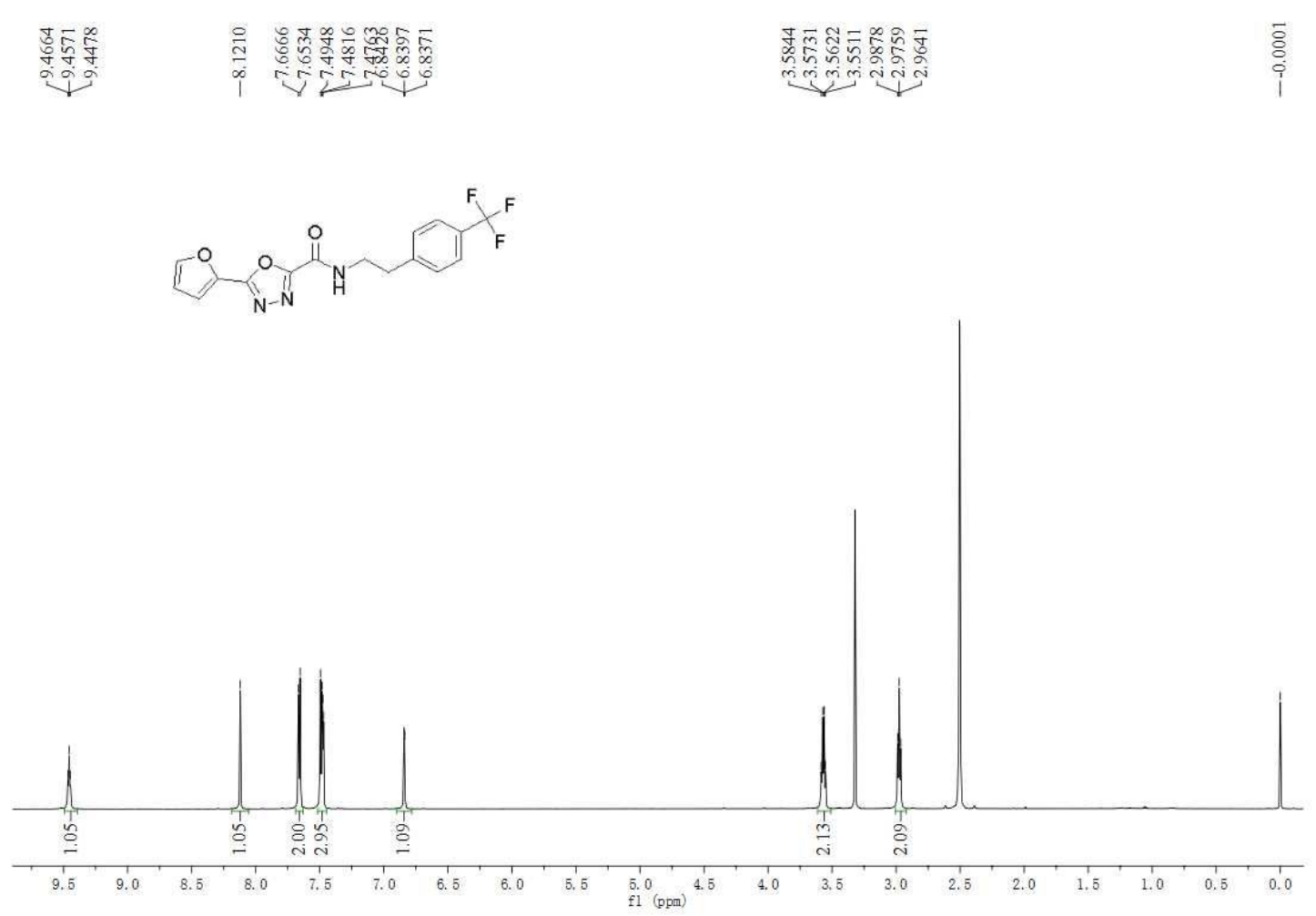

Figure S57. The ${ }^{1} \mathrm{H}$ NMR spectrum $\left(600 \mathrm{MHz}, \mathrm{DMSO}-d_{6}\right)$ of compound $\mathbf{5 l}$ 

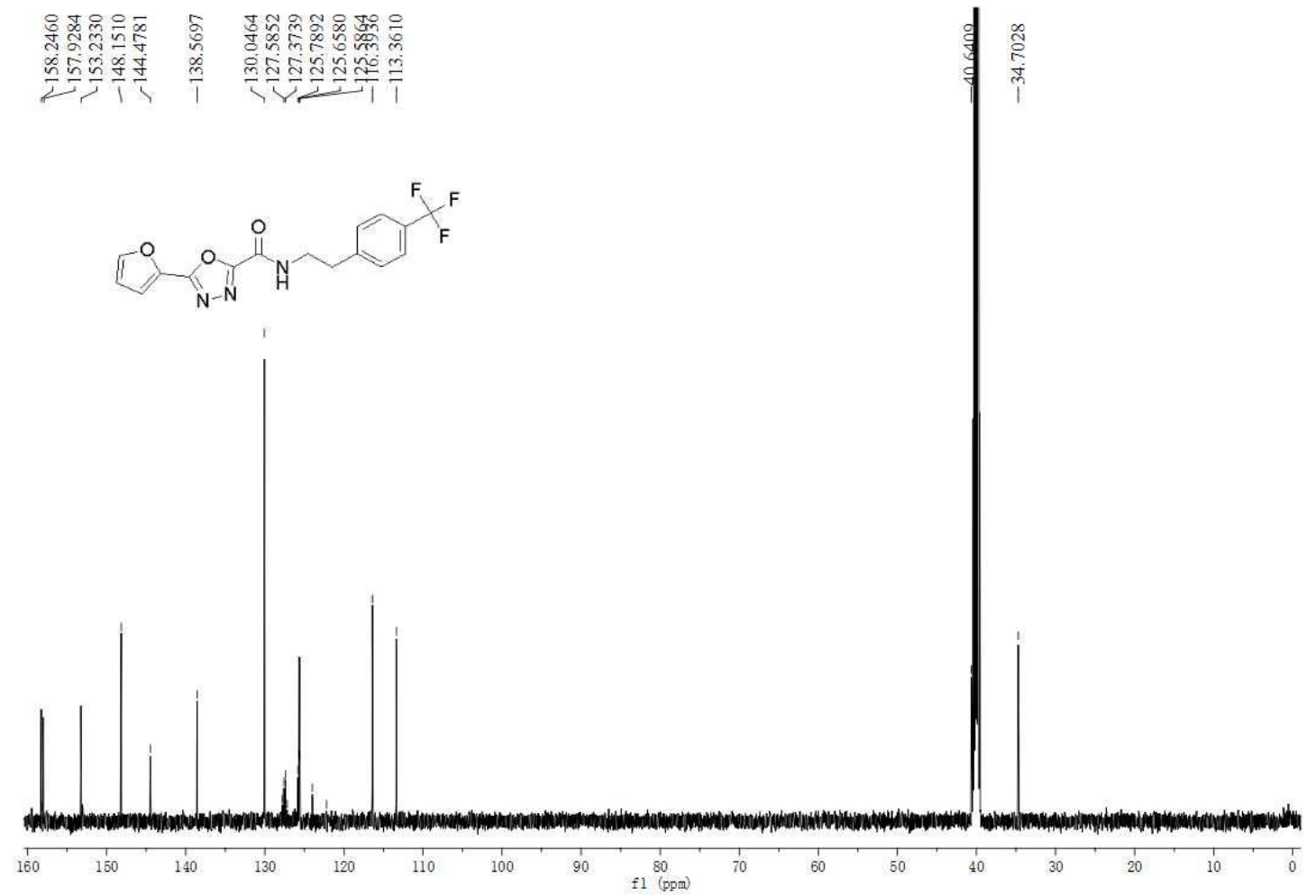

Figure S58. The ${ }^{13} \mathrm{C}$ NMR spectrum $\left(150 \mathrm{MHz}, \mathrm{DMSO}-d_{6}\right)$ of compound $\mathbf{5 l}$ 


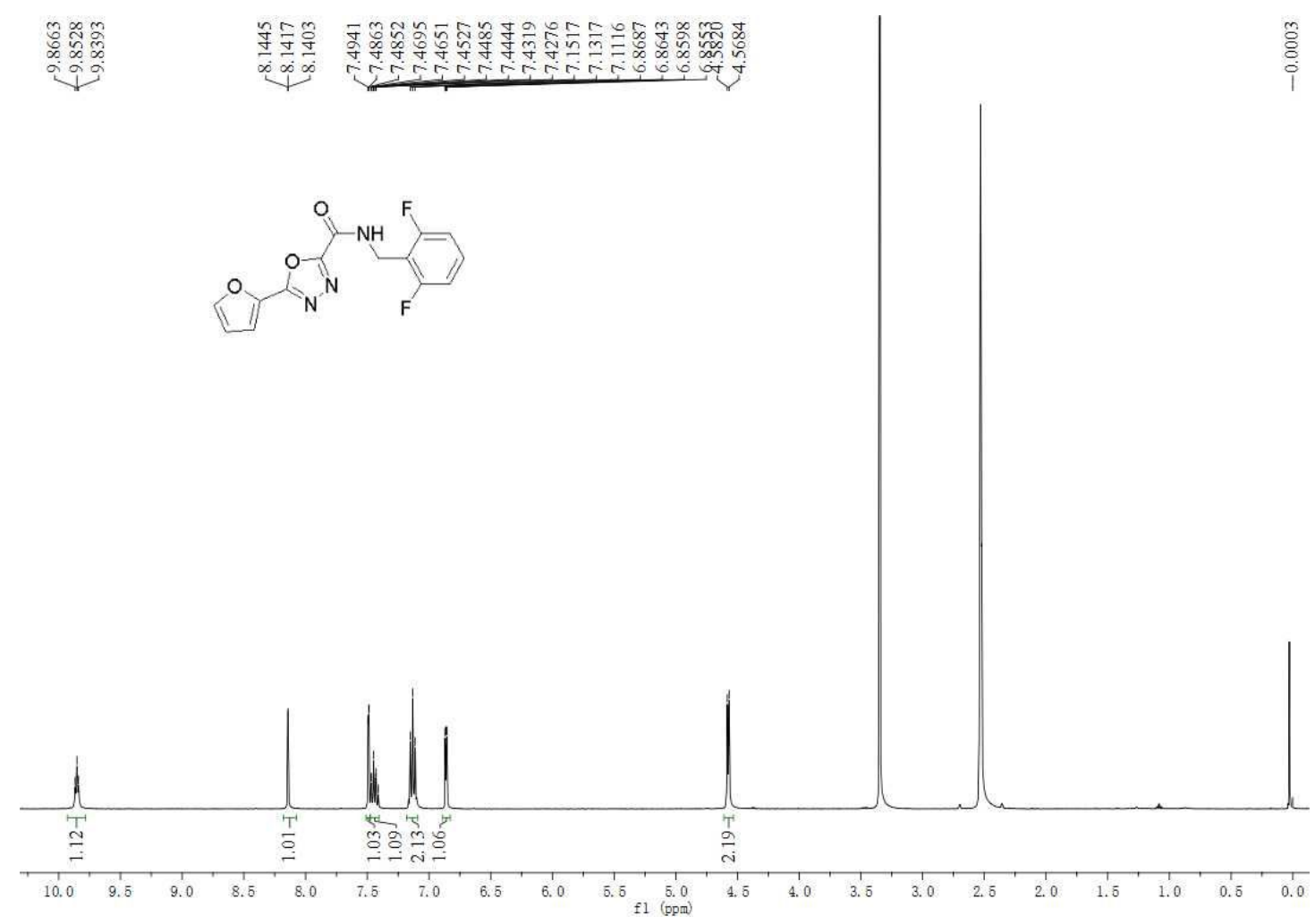

Figure S59. The ${ }^{1} \mathrm{H}$ NMR spectrum ( $\left.400 \mathrm{MHz}, \mathrm{DMSO}-d_{6}\right)$ of compound $\mathbf{5 m}$

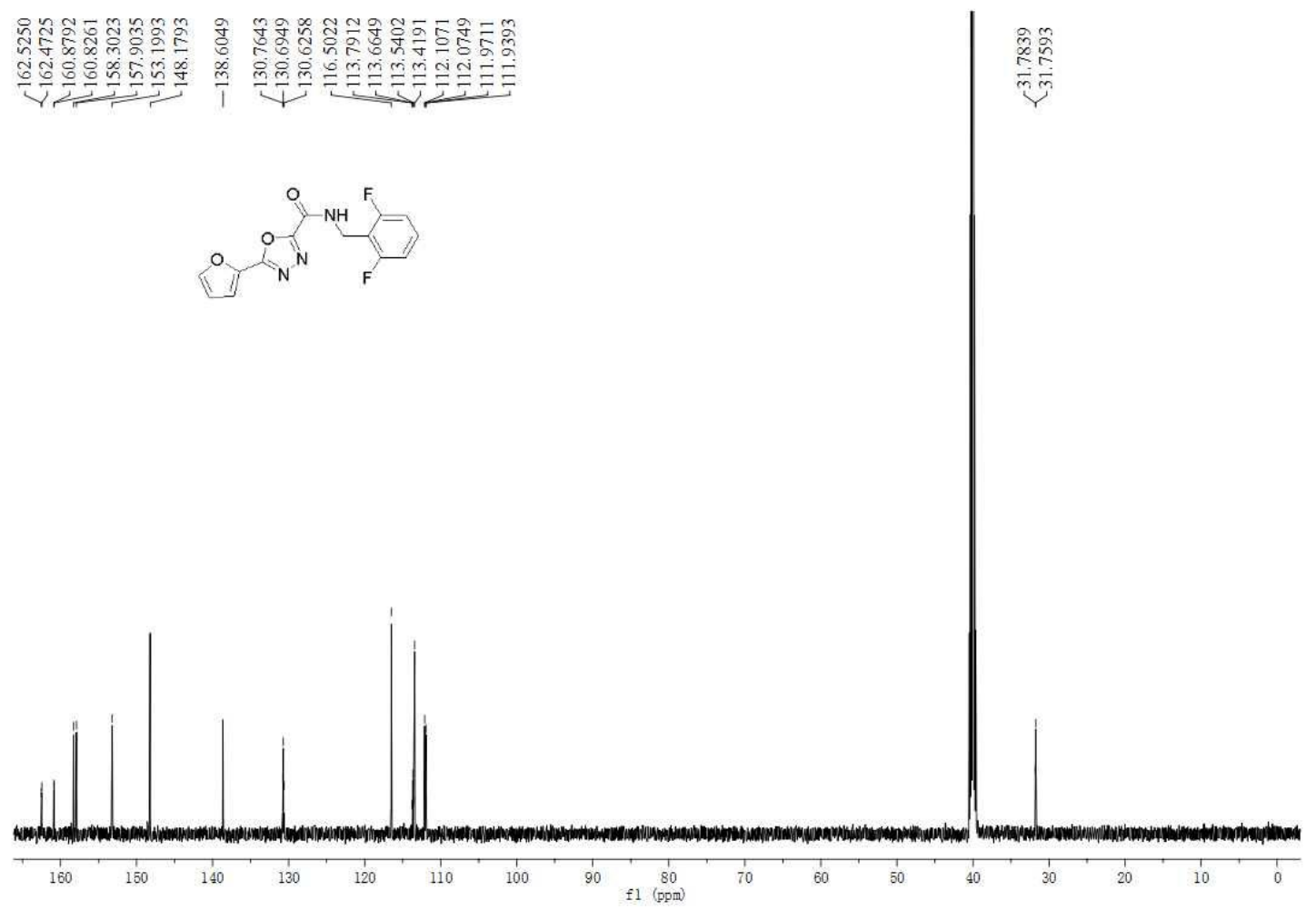

Figure S60. The ${ }^{13} \mathrm{CNMR}$ spectrum $\left(150 \mathrm{MHz}\right.$, DMSO- $\left.d_{6}\right)$ of compound $\mathbf{5 m}$ 


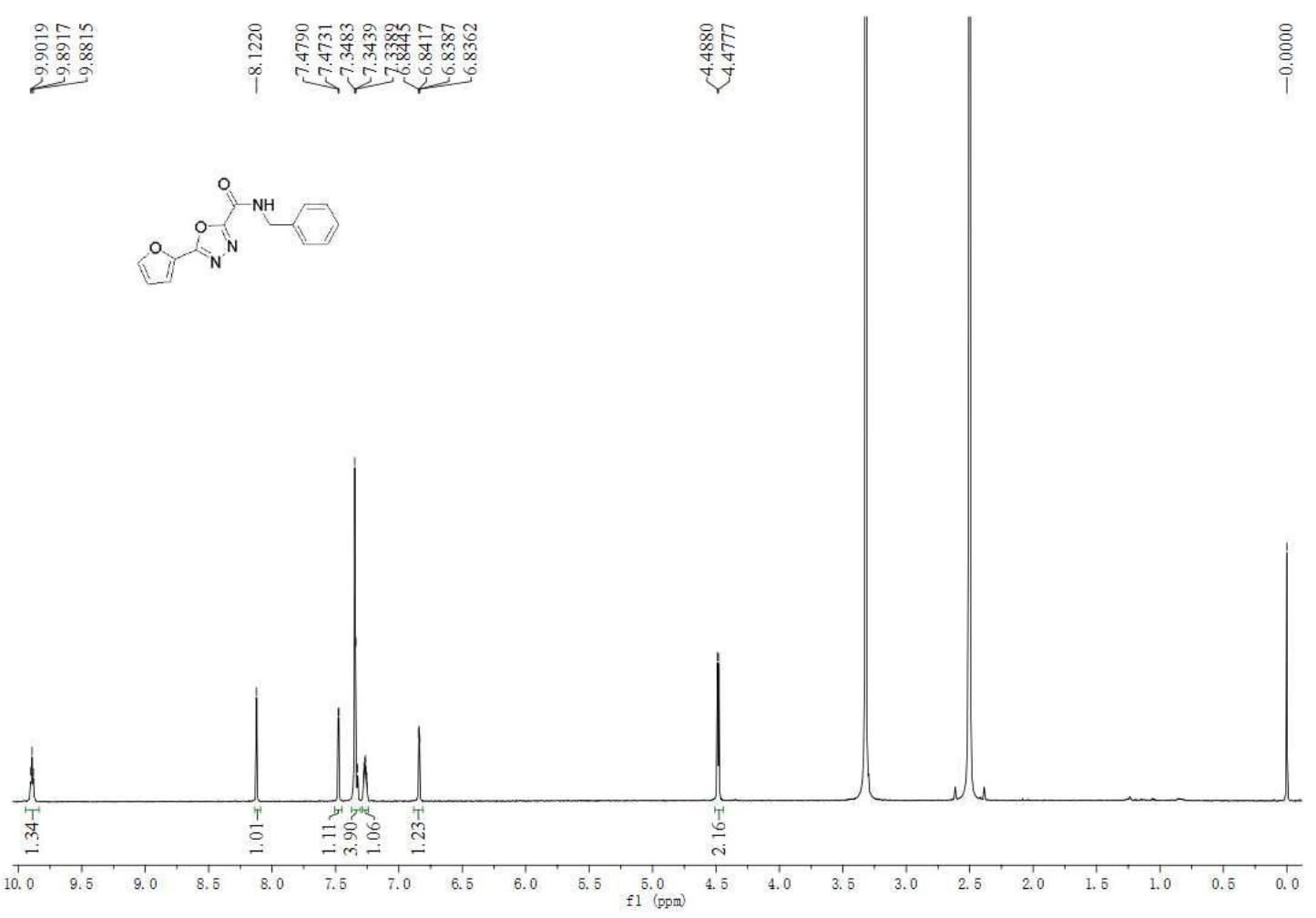

Figure S61. The ${ }^{1} \mathrm{H}$ NMR spectrum (600 MHz, DMSO- $\left.d_{6}\right)$ of compound $\mathbf{5 n}$

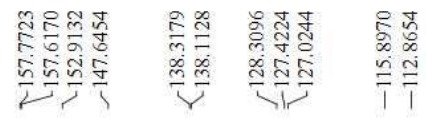<smiles>O=C(NCc1ccccc1)c1nnc(-c2ccco2)o1</smiles>

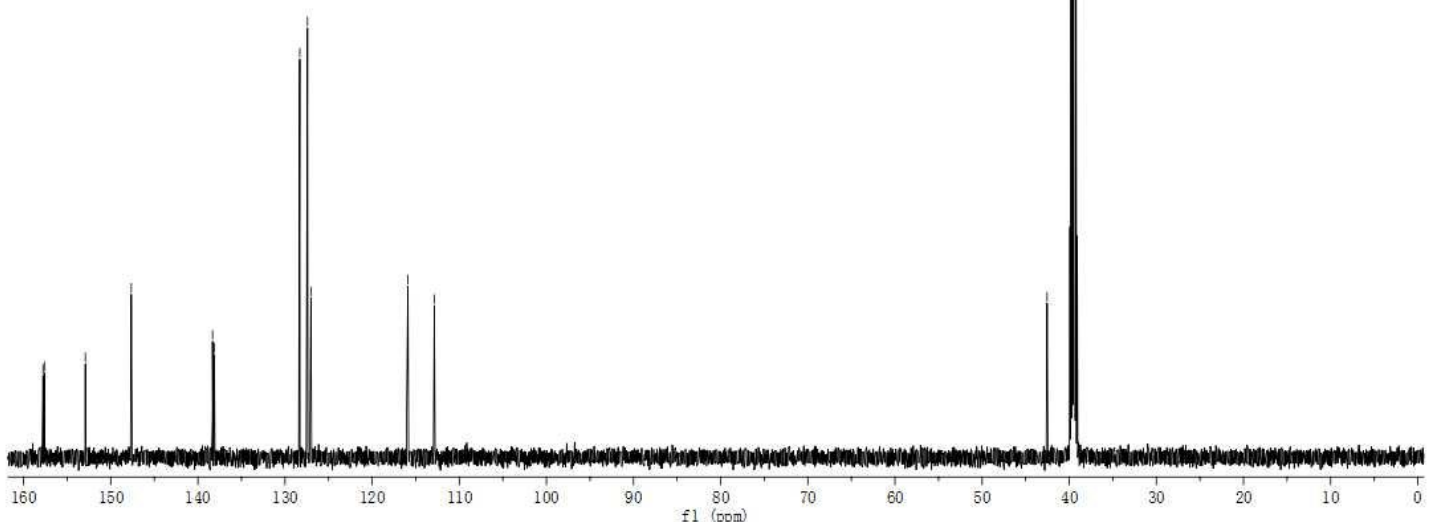

Figure S62. The ${ }^{13} \mathrm{C}$ NMR spectrum (150 MHz, DMSO- $d_{6}$ ) of compound $5 \mathbf{n}$ 


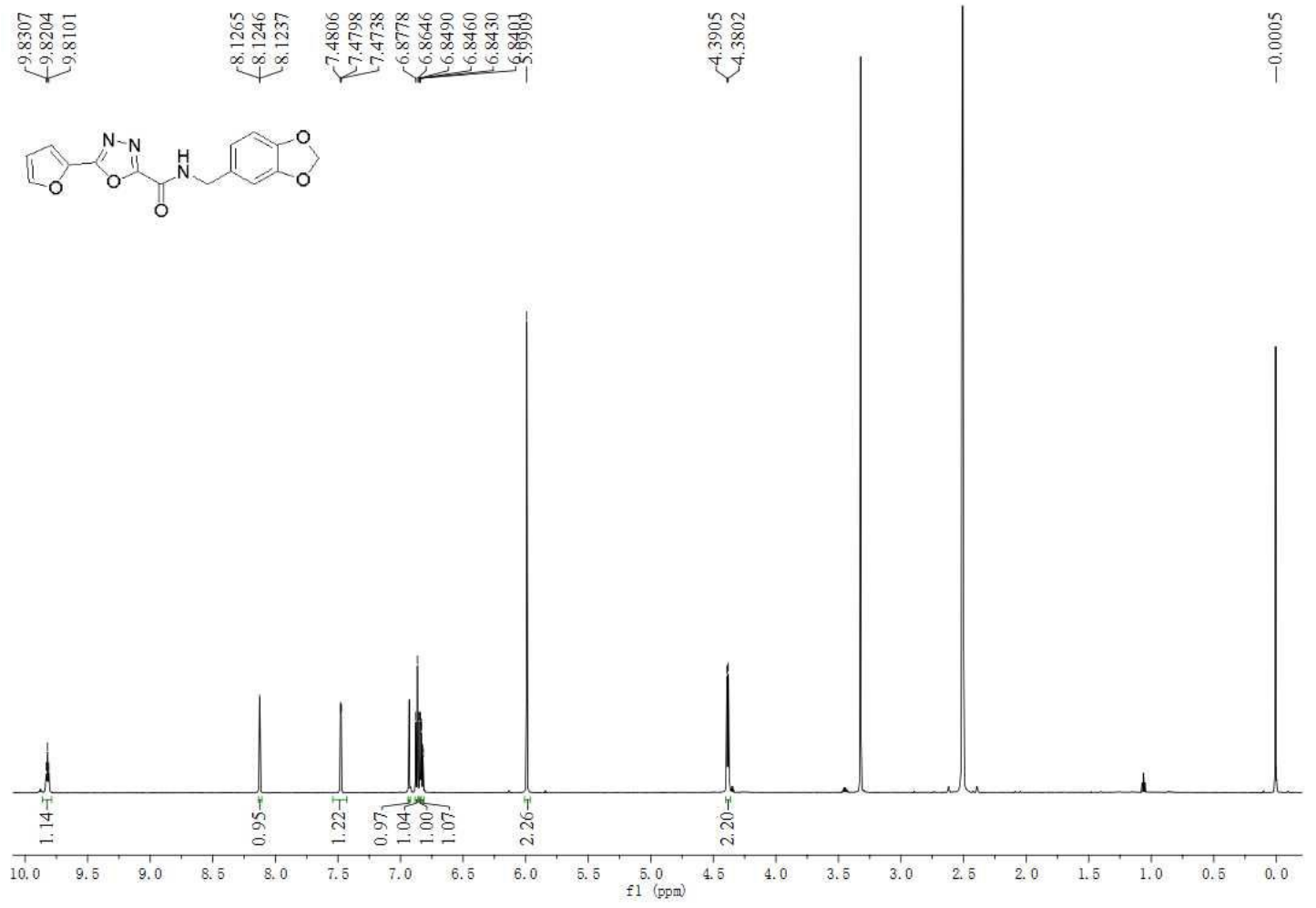

Figure S63. The ${ }^{1} \mathrm{H}$ NMR spectrum $\left(600 \mathrm{MHz}, \mathrm{DMSO}-d_{6}\right)$ of compound 50
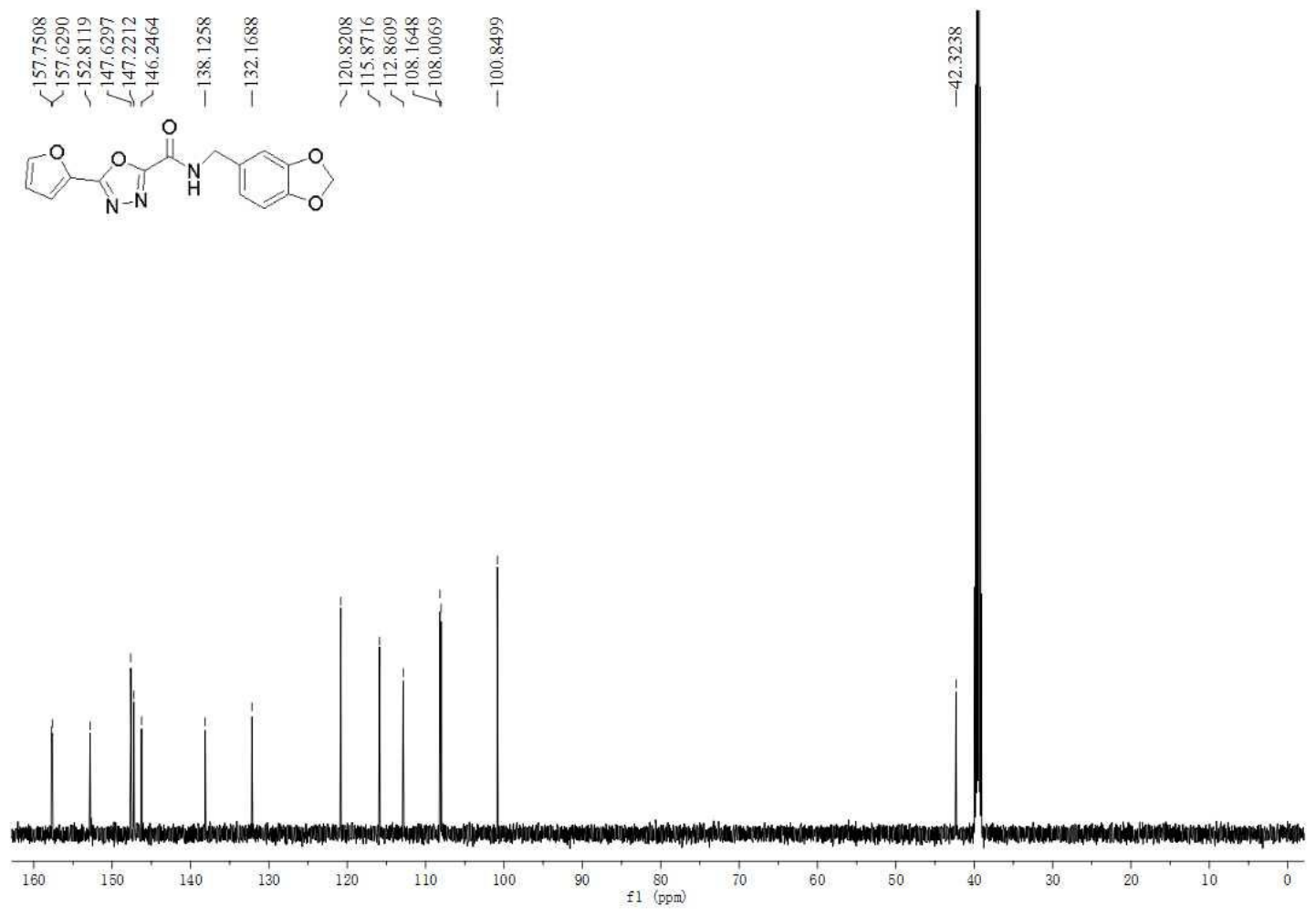

Figure S64. The ${ }^{13} \mathrm{C}$ NMR spectrum $\left(150 \mathrm{MHz}, \mathrm{DMSO}-d_{6}\right)$ of compound 50 
4. Crystallographic data of title compound 4g (Table S1).

\begin{tabular}{|c|c|}
\hline Compound & $4 g$ \\
\hline Empirical formula & $\mathrm{C}_{15} \mathrm{H}_{10} \mathrm{~F}_{3} \mathrm{~N}_{3} \mathrm{O}_{2} \mathrm{~S}$ \\
\hline Formula weight & 353.32 \\
\hline $\mathrm{T} / \mathrm{K}$ & 293(2) \\
\hline Radiation, $\lambda / \AA$ & $\mathrm{Cu} \mathrm{K} \alpha, 1.54178$ \\
\hline Crystal system, Space group & Triclinic, $P-1$ \\
\hline Unit cell dimensions / $\AA$ and ${ }^{\circ}$ & $a=5.6003(1), b=10.1540(3), c=13.8448(3)$ \\
\hline & $\alpha=105.521(2), \beta=92.920(2), \gamma=102.470$ (2) \\
\hline Volume / $\AA^{3}$ & $735.72(3)$ \\
\hline$Z$ & 2 \\
\hline Calculated density $/ \mathrm{g} \cdot \mathrm{cm}^{-3}$ & 1.595 \\
\hline Absorption coefficient $/ \mathrm{mm}^{-1}$ & 2.432 \\
\hline$F(000)$ & 360 \\
\hline Crystal size / mm & $0.20 \times 0.10 \times 0.10$ \\
\hline Theta range $/^{\circ}$ & 3.335 to 67.679 \\
\hline Index ranges & $-6 \leq h \leq 6,-12 \leq h \leq 12,-17 \leq l \leq 16$ \\
\hline Reflections collected & $16027\left(R_{\mathrm{int}}=0.0465\right)$ \\
\hline Independent reflections & 2850 \\
\hline Completeness to $\theta_{\max }$ & 0.957 \\
\hline Observed data / restraints / parameters & $2850 / 2 / 218$ \\
\hline Goodness-of-fit on $F^{2}$ & 1.010 \\
\hline Final $R$ indices $[I>2 \operatorname{sigma}(I)]$ & $R_{1}=0.0543$ \\
\hline \multirow{3}{*}{$R$ indices (all data) } & $w R_{2}=0.1589$ \\
\hline & $R_{1}=0.0549$ \\
\hline & $w R_{2}=0.1596$ \\
\hline Largest diff. peak and hole / e. $\AA^{-3}$ & 1.206 and -0.694 \\
\hline
\end{tabular}

